\title{
Ethnomedicinal knowledge of the rural communities of Dhirkot, Azad Jammu and Kashmir, Pakistan
}

\author{
Asia Farooq ${ }^{1}$, Muhammad Shoaib Amjad ${ }^{1}$, Khalid Ahmad ${ }^{2}$, Muhammad Altaf $^{3}$, Muhammad Umair ${ }^{4}$ and \\ Arshad Mehmood Abbasi ${ }^{2^{*}}$ id
}

\begin{abstract}
Background: Being an isolated locality and having a tough mountainous terrain, strong ethnomedicinal practices still prevail in Dhirkot and its allied areas, which have been rarely explored yet. The present study was intended with the aim to document and compare the traditional knowledge of local communities on botanical taxa of Dhirkot, Azad Jammu, and Kashmir.

Methodology: Ethnomedicinal data were collected from 74 informants using a semi-structured questionnaire in addition to field observation and group discussion. Various indices were also used to evaluate the ethnomedicinal data. Furthermore, the present findings were compared with previous reports to assess data novelty.

Result: A total of 140 medicinal plant species belonging to 55 families were recorded, which are used by local communities to treat 12 disease categories. Asteraceae was dominating with 20 species, followed by Poaceae, Lamiaceae, and Rosaceae $(14,11$, and 10 species, respectively). Herbs were leading with $66 \%$ contribution, whereas leaves were the most utilized plant part with $29 \%$ utilization and decoction was the common mode of administration. Viola canescens depicted the highest use value and relative frequency of citation ( 1.7 and 0.92 , respectively). Maximum informant consensus factor (0.88) was calculated for digestive and liver disorders. Five plant species including Berberis lycium Mentha arvensis Pyrus malus, Taraxacum officinale, and Viola canescens had 100\% fidelity level.

Conclusion: Dhirkot and its allied areas harbor rich botanical and cultural diversity because of its unique geography and diverse climatic conditions. However, mostly, traditional ethnobotanical knowledge is restricted to healers, midwives, and older people, and could be extinct in the near future. Therefore, such documentation not only conserves traditional knowledge but may also contribute significantly to novel drug resources.
\end{abstract}

Keywords: Traditional knowledge, Medicinal plants, FC, ICF, Dhirkot

\section{Background}

Medicinal plants are an important element of aboriginal curative systems. This knowledge is considered as a part of cultural assets [1] However, many indigenous groups fail to sustain and preserve this communal knowledge [2] that is why the systematic evaluation of this knowledge in order to contribute to health care in marginalized areas has been sighted in programs of national and international organizations [3]. In developing countries, most of the

\footnotetext{
*Correspondence: arshad799@yahoo.com; amabbasi@cuiatd.edu.pk ${ }^{2}$ Department of Environment Sciences, COMSATS University Islamabad, Abbottabad Campus 22060, Pakistan

Full list of author information is available at the end of the article
}

local communities are still relying on plant-based medicines [4]. The use of medicinal plants is a valuable source of income for poor communities but knowledge on therapeutic plants is decreasing gradually due to the progression in the present health care system and rapid urbanization $[5,6]$. Therefore, such rich tradition should be preserved through a reliable approach before it gets lost due to various anthropogenic and other causes.

There is an amazing growing interest in the alternative systems of therapeutics on a global level [7]. The factors contributing towards the potential use of herbal drugs in developing countries are accessibility, affordability, and historical and cultural background besides a holistic approach

(c) The Author(s). 2019 Open Access This article is distributed under the terms of the Creative Commons Attribution 4.0 International License (http://creativecommons.org/licenses/by/4.0/), which permits unrestricted use, distribution, and 
to health problems, safety, lack of adverse reaction, and side effects $[8,9]$. The use of plants as medicine ranges from 4 to $20 \%$ in different countries and about 2500 species are traded internationally. Pakistan has about 6000 species of higher plants, and among them, $10-30 \%$ of the flora is used for medicinal purposes in various areas $[10,11]$. The tradition of using medicinal plants in Pakistan for the treatment of various ailments is very mature, based predominantly on the Unani system of medicine. This traditional medicine sector has become an important source of health care, especially in rural and tribal areas of the country where it is considered as first-line treatment [12].

Azad Jammu and Kashmir (AJ\&K) is characterized by its diverse habitats, climate, and soil [13-16]. It is located in North-East of Pakistan and is stuffed with natural resources particularly plant flora [17]. AJK has a wide range of mountainous ecosystems which are affluent in fauna and flora. Due to extraordinary climatic conditions, the area has three vegetation groups (deserts, alpine, and grasslands). Natural and anthropogenic stresses have a great effect on the natural environment and ecosystems of the area [18]. Previously, different researchers reported ethnomedicinal uses of plant species from other parts of AJ\&K $[16,19,20]$. However, the present research area is rarely reported except in one study, which was conducted about 16 years ago [21]. We hypothesize that older people are more familiar with ethnomedicinal uses of plant species compared to younger people and formal education is not predictive of the traditional knowledge level of indigenous people. Moreover, among the local communities, having the same culture usage or importance of a plant species may vary. Therefore, the present study was designed to document the traditional knowledge of plant species and its quantitative assessment and to associate the frequency of occurrence with ethnomedicinal uses of plant species.

\section{Materials and methods Study area}

Dhirkot is a diversity-rich mountainous area of district Bagh, Azad Jammu, \& Kashmir, Pakistan. It is situated 55 $\mathrm{km}$ southeast of Muzaffarabad (the capital city for Azad Jammu and Kashmir) and $132 \mathrm{~km}$ from Islamabad. It is located on latitude $33^{\circ} 57^{\prime} \mathrm{N}$ and longitude $73^{\circ} 36^{\prime} \mathrm{E}$ (Fig. 1), covering an area of $150 \mathrm{~km}$ square with an altitudinal variation of $850-2200 \mathrm{~m}$ [22]. The climate of the study area is of a subtropical humid and moist temperate type with maximum precipitation occurring in July $(95 \mathrm{~mm})$ followed by August $(89 \mathrm{~mm})$. The weather remains pleasant in summer due to its location at high altitude. The hottest months are June and July with an average temperature of $24^{\circ} \mathrm{C}$ and $23^{\circ} \mathrm{C}$ respectively. Sometimes, the temperature rises to $29^{\circ} \mathrm{C}$. The coldest months are January and February with an average temperature of $5.3^{\circ} \mathrm{C}$ and $6.6^{\circ} \mathrm{C}$ respectively.
Sometimes, the temperature falls to $1.1{ }^{\circ} \mathrm{C}$, and at higher elevation, snowfall occurs (Fig. 2). The vegetation of the study area is subtropical humid and moist temperate type. The dominant tree species are Pinus roxburghii (Chir Pine) and $P$. wallichiana (Blue Pine). Due to the cool and humid condition, the vegetation is comprised of a wide variety of herbs, shrubs, and trees. The ground flora is composed of a number of angiosperms along with mosses and ferns.

The region embraced a diverse ethnic composition including Abbasi, Sudhans, Rajputs, and Gardazi. Among them, Abassi and Gardazi are the largest and well-settled tribes in the area. The whole population is Muslim. The majority of the population speaks the Hindko language, while Gojri and Urdu are also spoken. The major proportion of the indigenous community has very limited income sources. Majority of people are farmers, some people are job holders, some are labor, and few have their own business on a small scale. People also keep animals at their homes for livelihood. Few public health dispensaries are providing basic health facilities but people living at higher altitudes have limited access to them. They mainly depend on herbal remedies prepared at home or by traditional healers for primary health care.

\section{Sampling and plant identification}

Several field trips were made in four different seasons (from August 2017-July 2018) following the method as reported previously [23]. Each medicinal plant species was collected in triplicates from different localities during guided tours. The specimens were properly dried, pressed, and mounted on standard herbarium sheets and voucher specimens were prepared following Jain and Rao's methods [24]. Flora of Pakistan (https://www. eflora.com) $[25,26]$ was used for identification. For the correct family names, the APG IV (2016) [27] was followed, while for the accurate scientific name, "The Plant List (2013) [28] was used. The identified specimens were further confirmed in the AJ\&K Medicinal and Aromatic Plant Herbarium PARC, Pakistan. The fully identified voucher specimens were then deposited in the herbarium of the Women University of Azad Jammu \& Kashmir, Bagh.

\section{Data collection and analysis}

Ethnomedicinal data were gathered from 74 informants including male (55\%) and female (45\%) using semistructured interviews, questionnaire, group discussion, and field observation. The informants were selected on a random basis via convenience sampling and sample size was determined by Kadam and Bhalerao's method [29]. For the preparation of the questionnaire Edward et al. method was used [30]. And ethical guidelines of the International Society of Ethnobiology (http://www.ethnobiology.net/) were strictly followed. In this regard, ethical approval was taken 


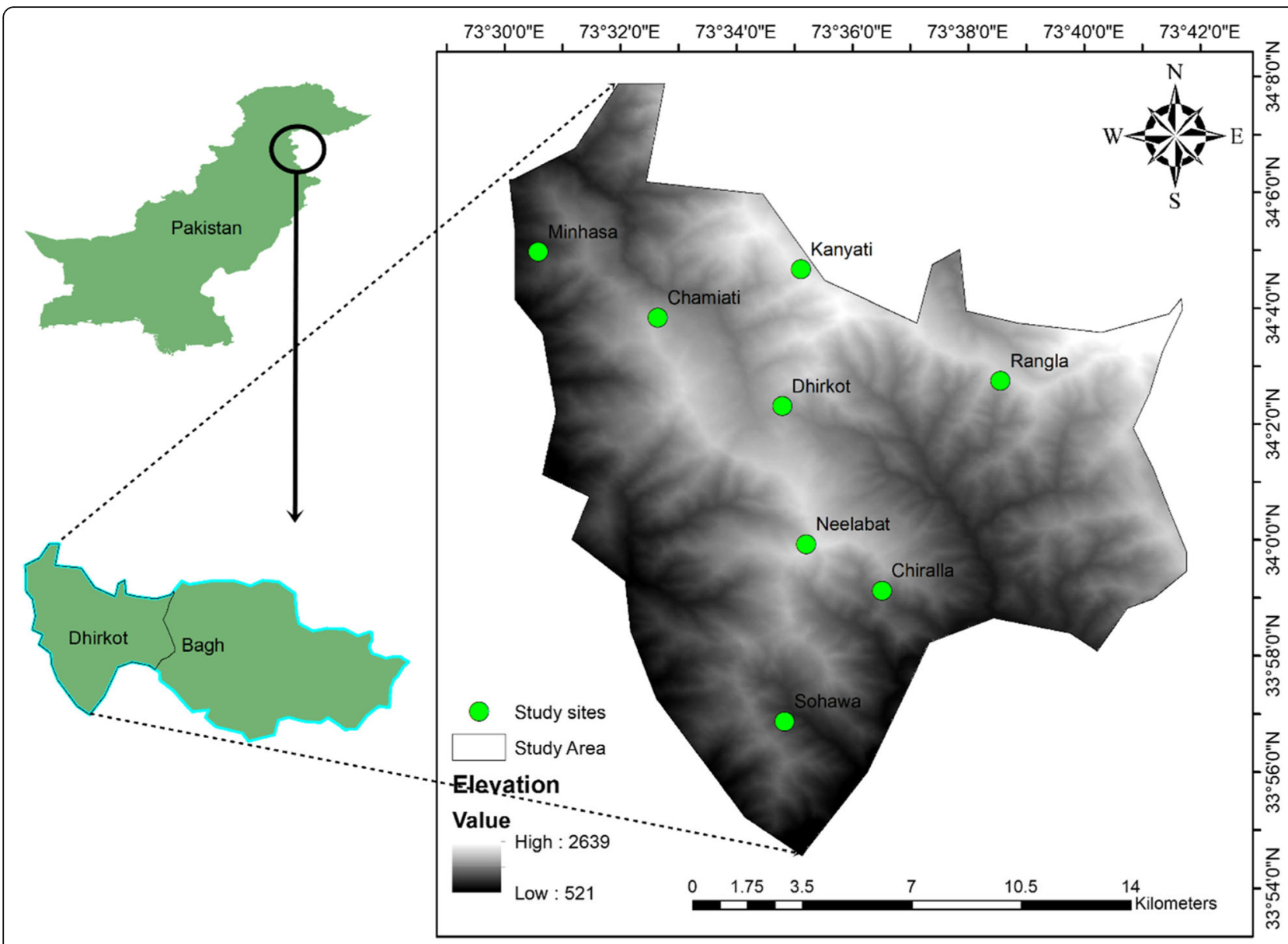

Fig. 1 Map of the study area

from the ethical committee of the Women University of Azad Jammu \& Kashmir before starting surveys, while legal permission for conducting the survey was also taken from the representative of the municipality. Prior consent was taken from all the respondents following the participatory rural appraisal (PRA) approach as mentioned in the Kyoto Protocol after explaining the possible objective consequences of the study in the local language. Informants were not subjected to any clinical trial. Informants were classified into different categories like age, education level, and professions. The correctness of the ethnobotanical data was checked through triangulation. The data was then compared with the existing literature and analyzed both quantitatively and qualitatively.

\section{Ethnobotanical indices}

For quantitative analysis various quantitative indices were applied including;

\section{Relative frequency citation}

The frequency of citation (FC) was used to identify the most used plant species by the local inhabitants of the area. It was calculated by following Tardio and Pardo-de Santayana [5] and Vitalini et al. [31], using the following formula:

$$
\mathrm{RFC}=\frac{\mathrm{FC}}{\mathrm{N}}
$$

where FC is respondents citing the use of specific species and $N$ are the total respondents.

\section{Use value}

The relative importance of particular plant species cited by all informants in a given area is quantitatively measured in terms of the use value. It was calculated by following Savikin et al. [32] using the following formula:

$$
\mathrm{UV}=\frac{\sum \mathrm{Ui}}{N}
$$

where $\mathrm{Ui}$ is the number of citations or used reports by each respondent for a particular plant species and $N$ is the total respondents. 


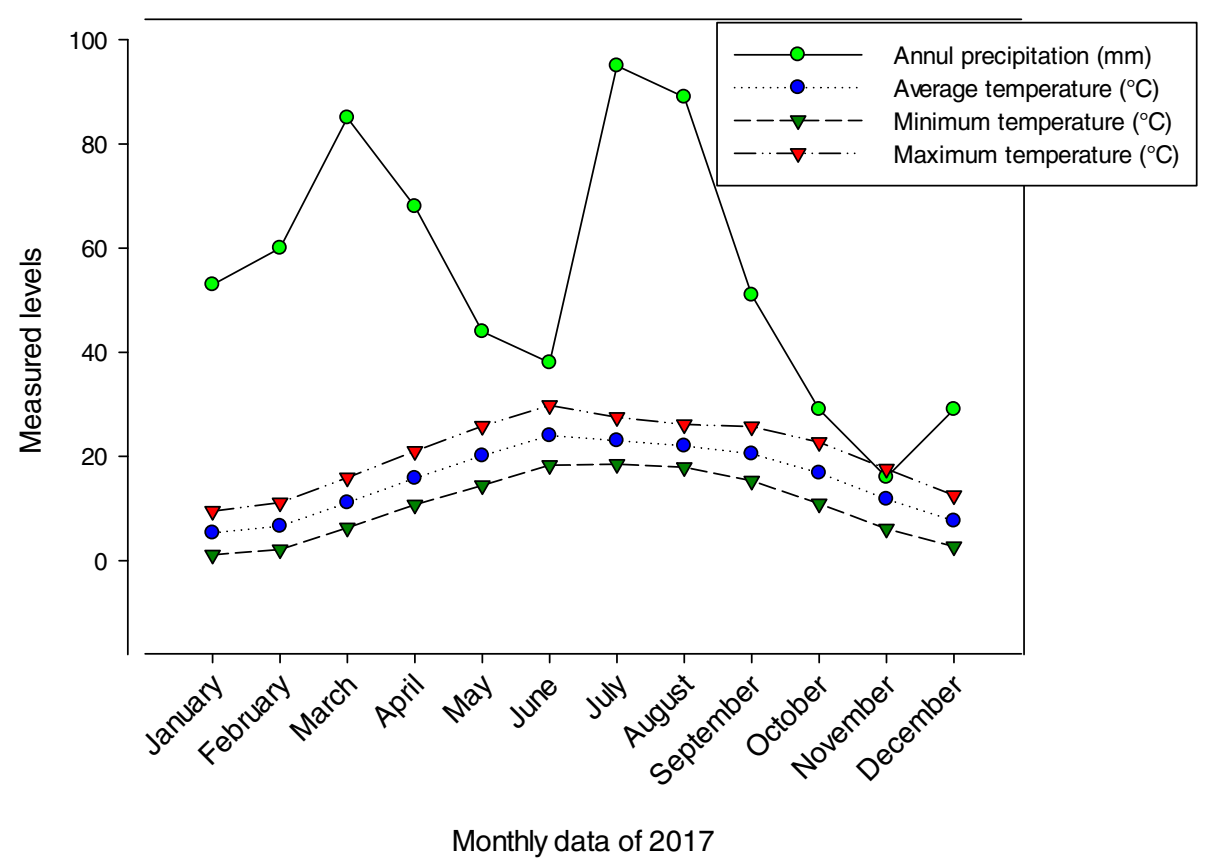

Fig. 2 Precipitation and temperature data of the study area for the year 2017

\section{Informant consensus factor}

The consensus between respondents and particular plant species used for each diseased category was tested by using informant consensus factor. It was figured out by following Vitalini et al., [5] using given formula:

$$
\text { A.ICF }=\frac{\text { Nur-Nt }}{(\mathrm{Nt}-1)}
$$

where 'Nur' represents the total number of used reports in each group of diseases, and ' $\mathrm{Nt}$ ' represents the total species cited by all the informants for that group of ailments.

\section{Jaccard index}

The similarity of indigenous knowledge among different communities was determined by using the Jaccard index (JI). It was calculated by following Gonzalez-Tejero et al. [33] using the given formula:

$$
\mathrm{JI}=\frac{\mathrm{C} \times 100}{(\mathrm{a}+\mathrm{b})-\mathrm{c}}
$$

where $a$ is the species of the study area, $b$ is the species recorded from the allied area, and $c$ is the common species in both areas.

\section{Relative importance}

Relative importance (RI) was figured out by following Khan et al. [34] using the given formula.

$$
\operatorname{Re} 1 \mathrm{PH}=\frac{\mathrm{RI}=(\operatorname{RelPH}+\operatorname{RelBS}) \times \frac{100}{2}}{\text { Maximum } \text { PH a givn plant reported plant species }}
$$

where $\mathrm{PH}$ is the pharmacological attribute of the selected plants and Rel $\mathrm{PH}$ is the relative number of pharmacological properties attributed to individual plant species.

$$
\text { Re1BS }=\frac{\text { BS of a given plant }}{\text { Maximum BS of all reported plant species }}
$$

BS is the number of body systems healed up by using single species and Rel BS is the relative number of body systems healed up by using a single species.

\section{Fidelity level}

The fidelity level (FL) index was used to determine the most preferred species used to cure a particular disease as to treat the same ailment category with more than one plant species is also used. It was figured out after Friedman et al. [35], using the given formula:

$$
\mathrm{FL}=\frac{N p}{N} \times 100
$$

where $N p$ is the number of respondents citing the use of species for a particular ailment and $N$ is the total number of respondents citing the plants for any illness. 


\section{Results and discussion}

\section{Medicinal plants use and knowledge variation}

The data on medicinal uses of plants was collected from 12 villages. Detail demographic data is given in Table 1. The females usually avoid participating and sharing knowledge with male interviewee due to communal restriction and Islamic instruction, which is also mentioned in other studies [36-38]. However, the women hold a wider competence regarding the traditional herbal recipes $(5.36 \%$ species; $8.68 \%$ uses). A similar trend was also observed in other studies from Pakistan and abroad [39-41]. The older people (age $\leq 60)$ have more knowledge $(6.46 \%$ species; $10.82 \%$ uses), followed by middle-aged people (age $\leq$ 40) (6.34\% species; $9.50 \%$ uses) in comparison to adolescent informants (age $\leq 19$ ) while it is inversely proportional to the level of education (Table 1). This might be the consequence of modernization and weak beliefs of young people regarding traditional remedies and due to changing lifestyles, development in modern medication, and urbanization [42, 43]. Similar findings are reported from other areas of Pakistan [44, 45] and elsewhere [4648]. Illiterate native people are more accustomed to the usage of ethnomedicinal plants than literate people. The reason behind this is that educated people have very less

Table 1 Demographic information of the Informants

\begin{tabular}{|c|c|c|c|c|}
\hline Variables & IC & Number & ANSRI & ANURI \\
\hline \multirow[t]{3}{*}{ Gender } & Male & 41 & 4.53 & 7.71 \\
\hline & Female & 33 & 5.36 & 8.68 \\
\hline & Total & 74 & & \\
\hline \multirow[t]{3}{*}{ Age-Class } & $19-40$ & 17 & 4.17 & 3.46 \\
\hline & $41-60$ & 44 & 9.34 & 5.23 \\
\hline & Above 60 & 13 & 13.1 & 11.7 \\
\hline \multirow[t]{6}{*}{ Education Level } & Illiterate & 12 & 6.59 & 4.23 \\
\hline & Elementary education & 16 & 13.7 & 6.40 \\
\hline & Secondary education & 18 & 13.1 & 6.02 \\
\hline & HSE & 14 & 6.40 & 5.70 \\
\hline & Bachelor degree & 9 & 17.1 & 4.92 \\
\hline & Higher education & 5 & 11.5 & 6.91 \\
\hline \multirow[t]{9}{*}{ Professions } & THPS & 12 & 21.5 & 10.4 \\
\hline & Midwives & 07 & 12.4 & 7.36 \\
\hline & Herders & 05 & 10.2 & 8.33 \\
\hline & Housewives & 15 & 7.88 & 6.31 \\
\hline & Teachers & 8 & 7.29 & 8.54 \\
\hline & Farmers & 14 & 5.65 & 4.40 \\
\hline & Shopkeeper & 04 & 4.18 & 3.98 \\
\hline & Students & 06 & 4.31 & 3.04 \\
\hline & Labors & 03 & 5.23 & 4.75 \\
\hline
\end{tabular}

IC informants category, ANSRI average number of species reported by each informant, ANURI average number of use reported by each informant, HSE higher secondary education, THPs traditional health practitioners interest in learning and practicing ethnobotanical knowledge. The same result was documented by other researchers in Pakistan $[20,49-51]$ and abroad [52, 53].

\section{Local health care system}

Throughout history, the role of traditional health practitioners (THPs) and midwives varies with time and culture, but even today, they are contributing significantly to the primary health care system, particularly among marginalized communities. THPs are usually aged males that use plants, animals, and minerals to treat various health disorders, whereas midwives are the elders and experienced females, which are familiar with pregnancy issues of women and treat them using diverse medicinal plants. Midwives are the integral component of a community that perform their important duties and provide essential support to women during delivery $[54,55]$. Data given in Table 1 revealed that most of the information on ethnomedicinal uses of plant species of the study area were shared by (THPs), and midwives. The average number of species reported by THPs and midwives was 21.5 and 12.4, while they reported about $10.4 \%$ and $7.36 \%$ uses in respective order. Most of the traditional health practitioners were males who possess extensive information about therapeutic herbs and natural treatments which they use in herbal and other remedial preparations to cure diseases $[56,57]$. However, as reported previously, traditional knowledge of plant resource utilization is declining due to changing lifestyle and more dependence on allopathic medicines [20, 51, 58, 59]. And similar trends were noted in the study areas.

\section{Diversity of ethnomedicinal flora}

A total of 140 species belonging to 55 families and 93 genera were reported (Table 2). Most of the documented ethnomedicinal plants species were herbs $(66 \%)$ followed by shrubs (16\%), trees (14\%), and climbers (4\%), (Fig. 3). This is because the study area is located in a dense forest zone at higher altitude where the herbs are abundantly distributed with few trees and shrubs. The bimodal rainfall and high availability of moisture might also be the reason. These findings are consistent with other studies [62-65, 69, 75, 76]. Among 22 families representing 2-20 plant species (Fig. 4), Asteraceae was the dominant family with $14.29 \%$ contribution of the total reported taxa, followed by Poaceae (10\%), Lamiaceae (7.86\%), Rosaceae (7.14\%), Fabaceae (4.29\%), and Pteridaceae (3.57\%). All other families contributed less than $5 \%$ with percentages varying from $0.71-2.86 \%$. The dominance of Asteraceae, Poaceae, Lamiaceae, and Rosaceae might be due to suitable habitat, favorable environmental conditions for the growth of the species belonging to these families, and more interactions of local communities with them in the study area. Therefore, traditional uses of plant species of these species are well recognized by the local inhabitants $[6,36,66,77,78]$. Additionally, majority of plant 
Table 2 Medicinal uses of the reported taxa and their comparison with previous reports

\begin{tabular}{|c|c|c|c|c|c|c|c|c|c|}
\hline \multirow{2}{*}{$\begin{array}{l}\text { Sr } \\
\#\end{array}$} & \multirow[t]{2}{*}{ Family } & \multicolumn{2}{|l|}{ Nomenclature } & \multirow[t]{2}{*}{ Habit } & \multicolumn{4}{|c|}{ Medicinal uses } & \multirow[t]{2}{*}{ Previous reports } \\
\hline & & Scientific name & Local name & & $\begin{array}{l}\text { Part } \\
\text { used }\end{array}$ & Preparation & Application & Disease treated & \\
\hline \multirow[t]{5}{*}{1} & \multirow[t]{5}{*}{ Acanthaceae } & \multirow{2}{*}{$\begin{array}{l}\text { Dicliptera } \\
\text { roxburghiana } \\
\text { Nees./AF-110 }\end{array}$} & \multirow[t]{2}{*}{ Churun } & \multirow[t]{2}{*}{$\mathrm{H}$} & WP & PD & Internal & *Diabetes, *Tonic & \multirow{2}{*}{$\begin{array}{l}1 \bullet, 2 \bullet, 3 \bullet, 4 \bullet, 5 \bullet, 6 \bullet, \\
7 \quad, 8 \bullet, 9 \bullet, 10 \bullet, 11 \bullet, 12 \bullet, \\
13 \bullet, 14 \bullet, 15 \bullet, 16 \bullet, 17 \bullet, \\
18 \bullet, 19 \bullet, 20 \bullet, 21 \bullet, 22 \bullet\end{array}$} \\
\hline & & & & & RT & EX & External & *Wounds & \\
\hline & & $\begin{array}{l}\text { Justicia vahlii } \\
\text { Roth./AF-9 }\end{array}$ & Bhekkar & $\mathrm{H}$ & LF & IN & Internal & $\begin{array}{l}\text { Respiratory tract } \\
\text { diseases }\end{array}$ & $\begin{array}{l}1 \bullet, 2 \bullet, 3 \bullet, 4 \bullet, 5 \bullet, 6 \bullet, 7 \bullet, \\
8 \bullet, 9 \bullet, 10 \bullet, 11 \bullet, 12 \bullet, 13 \bullet, \\
14 \bullet, 15 \bullet, 16 \bullet, 17 \bullet, 18 \bullet, \\
19 \bullet, 20 \bullet, 21 \bullet, 22 \bullet\end{array}$ \\
\hline & & \multirow{2}{*}{$\begin{array}{l}\text { Pteracanthus } \\
\text { urticifolius (Wall. } \\
\text { ex Kuntze) } \\
\text { Bremek. /AF-48 }\end{array}$} & \multirow[t]{2}{*}{ Blue Nettle } & \multirow[t]{2}{*}{$\mathrm{H}$} & \multirow[t]{2}{*}{ WP } & EX & Internal & $\begin{array}{l}\text { Diuretic, Stomach } \\
\text { disorders, Ulcer }\end{array}$ & \multirow{2}{*}{$\begin{array}{l}1 \bullet, 2 \bullet, 3 \bullet, 4 \bullet, 5 \bullet, 6 \bullet, 7 \bullet, \\
8 \bullet, 9 \bullet, 10 \bullet, 11 \bullet, 12 \bullet, \\
13 \bullet, 14 \bullet, 15 \bullet, 16 \bullet, 17 \bullet, \\
18 \bullet, 19 \bullet, 20 \bullet, 21 \bullet, 22 \bullet\end{array}$} \\
\hline & & & & & & DE & Internal & Sedative, Tonic & \\
\hline \multirow[t]{2}{*}{2} & \multirow[t]{2}{*}{ Adoxaceae } & \multirow{2}{*}{$\begin{array}{l}\text { Viburnum } \\
\text { grandiflorum } \\
\text { Wall. ex DC./AF- } \\
92\end{array}$} & \multirow[t]{2}{*}{ Guch } & \multirow[t]{2}{*}{ S } & SD & JU & Internal & Typhoid & \multirow{2}{*}{$\begin{array}{l}1 \bullet, 2 \bullet, 3 \bullet, 4 \bullet, 5 \bullet, 6 \\
7,8 \bullet, 9 \bullet, 10 \bullet, 11 \bullet, 12 \bullet, \\
13 \bullet, 14 \bullet, 15 \bullet, 16 \bullet, 17 \bullet, \\
18 \bullet, 19 \bullet, 20 \bullet, 21 \bullet, 22 \bullet\end{array}$} \\
\hline & & & & & FR & ET & Internal & *Stomachache & \\
\hline \multirow[t]{11}{*}{3} & \multirow[t]{11}{*}{ Amaranthaceae } & \multirow{4}{*}{$\begin{array}{l}\text { Achyranthes } \\
\text { aspera L./AF-7 }\end{array}$} & Puthkanda & $\mathrm{H}$ & LE & DE & External & *Toothache & $1 \bullet, 2,3,4 \bullet, 5,6 \bullet$ \\
\hline & & & & & RT & EX & External & *Earache & $\begin{array}{l}7 \bullet, 8 \bullet, 9,10 \bullet, 11 \bullet, 12 \\
13 \bullet, 14,15 \quad, 16 \bullet, 17 \bullet,\end{array}$ \\
\hline & & & & & WP & DE & Internal & *Pneumonia & $18 \bullet, 19,20 \bullet, 21 \quad, 22$ \\
\hline & & & & & & EX & Internal & Dysentery & \\
\hline & & Amaranthus & Ganyar & $\mathrm{H}$ & LE & VG & Internal & Constipation & $1 \bullet, 2,3 \bullet, 4 \bullet, 5 \quad, 6 \bullet$ \\
\hline & & & & & ST & VG & Internal & Cough & $\begin{array}{ll}1 \bullet, 8 \bullet, 9 & , 10 \bullet, 11 \bullet, \\
12,13,14 \bullet, 15 \bullet, 16\end{array}$ \\
\hline & & & & & SD & PD & Internal & Eye Vision & $\begin{array}{l}17 \bullet, 18 \bullet, 19,20 \bullet, 21 \quad \text {, } \\
22 \bullet\end{array}$ \\
\hline & & $\begin{array}{l}\text { Chenopodium } \\
\text { ambrosioides } \mathrm{L} \text {. }\end{array}$ & Bathu/Bathwa & $\mathrm{H}$ & WP & IN & Internal & $\begin{array}{l}\text { *Measles, }{ }^{*} \text { Cough, } \\
\text { Amenorrhea }\end{array}$ & $\begin{array}{l}1 \bullet, 2 \bullet, 3 \bullet, 4 \bullet, 5 \bullet, 6 \bullet, 7 \bullet \\
8 \bullet, 9 \bullet, 10 \bullet, 11,12,\end{array}$ \\
\hline & & /AF-84 & & & LE & PA & External & *Joint pain, *Backache & $\begin{array}{ll}13 & , 14 \bullet, 15 \bullet, 16 \bullet, 17 \bullet \\
18 & , 19 \bullet, 20 \bullet, 21\end{array}$ \\
\hline & & & & & & PD & Internal & ${ }^{*}$ Cough, ${ }^{*}$ Motion & \\
\hline & & & & & SD & PD & Internal & $\begin{array}{l}\text { *Diuretic, *Dropsy } \\
\text { (oedema) }\end{array}$ & \\
\hline 4 & Apocynaceae & Nerium oleander & Kneer & S & LF & $\mathrm{CH}$ & External & Mouth disease & $1 \bullet, 2,3 \bullet, 4 \bullet, 5 \quad, 6 \bullet$ \\
\hline & & L. /AF-40 & & & RT & PA & External & Scorpion bite & $\begin{array}{l}7 \bullet, 8 \bullet, 9 \bullet, 10 \bullet, 11 \bullet, 12 \bullet \\
13 \bullet, 14 \quad, 15 \bullet, 16 \bullet, 17 \bullet\end{array}$ \\
\hline & & & & & BA & EX & External & To kill wound worms & $18 \bullet, 19 \bullet, 20 \bullet, 21 \quad, 22$ \\
\hline 5 & Araliaceae & Hedera & Hurrbumbal/ & E & LF & $\mathrm{DE}$ & Internal & Diabetes & $1,2 \bullet, 3 \bullet, 4 \bullet, 5 \bullet, 6 \bullet, 7 \bullet$, \\
\hline & & $\begin{array}{l}\text { nepalensis K. } \\
\text { Koch. /AF-135 }\end{array}$ & Betkal & & & נU & Internal & *Indigestion, Ulcer & $\begin{array}{l}8 \bullet, 9 \bullet, 10,11 \bullet, 12 \\
13 \bullet, 14 \bullet, 15 \quad, 16 \bullet, 17 \bullet\end{array}$ \\
\hline & & $\begin{array}{l}\text { Hydrocotyle spp. } \\
\text { L. /AF-114 }\end{array}$ & $\begin{array}{l}\text { Chamk wali } \\
\text { boti }\end{array}$ & $\mathrm{H}$ & LF & EX & Internal & $\begin{array}{l}\text { Fever, Bowel } \\
\text { Complaints }\end{array}$ & \\
\hline & & & & & & EX & External & Cuts, Burns & $1 \bullet, 2 \bullet, 3 \bullet, 4 \bullet, 5 \bullet, 6 \bullet, 7 \bullet$, \\
\hline & & & & & & $\mathrm{PO}$ & External & Syphilitic ulcers & $\begin{array}{l}8 \bullet, 9 \bullet, 10 \bullet, 11 \bullet, 12 \bullet, 13 \bullet, \\
14 \bullet, 15 \bullet, 16 \bullet, 17 \bullet, 18 \bullet,\end{array}$ \\
\hline & & & & & WP & $\mathrm{DE}$ & Internal & Influenza, Hepatitis & $19 \bullet, 20 \bullet, 21 \bullet, 22 \bullet$ \\
\hline 6 & Aspleniaceae & Asplenium & Niaroi & $\mathrm{H}$ & WP & JU & External & Blisters & $1 \bullet, 2 \bullet, 3 \bullet, 4 \bullet, 5 \bullet, 6 \bullet, 7 \bullet$, \\
\hline & & $\begin{array}{l}\text { dalhousiae } \\
\text { Hook. /AF-13 }\end{array}$ & & & & & Internal & Cough & $\begin{array}{l}6 \bullet, 8 \bullet, 9 \bullet, 10 \bullet, 11 \bullet, 12 \bullet, \\
13 \bullet, 14 \bullet, 15 \bullet, 16 \bullet, 17 \bullet,\end{array}$ \\
\hline & & & & & LE & EX & External & Swelling, Rickets & $18 \bullet, 19 \bullet, 20 \bullet, 21 \bullet, 22 \bullet$ \\
\hline 7 & Asteraceae & $\begin{array}{l}\text { Achillea } \\
\text { millefolium L. }\end{array}$ & $\begin{array}{l}\text { Sultani Booti / } \\
\text { Kangi Booti }\end{array}$ & $\mathrm{H}$ & $\mathrm{FL}$ & EX & Internal & $\begin{array}{l}\text { *Common Cold, *Flue, } \\
{ }^{*} \text { Cough }\end{array}$ & $\begin{array}{l}1,2 \bullet, 3,4,5 \bullet, 6 \bullet, \\
7 \bullet, 8,9 \bullet, 10 \bullet, 11 \bullet, 12 \bullet\end{array}$ \\
\hline & & & & & & & External & *Arthritis & $\begin{array}{l}13 \bullet, 14 \bullet, 15 \bullet, 16 \bullet, 1 \bullet \bullet \\
18 \bullet, 19 \bullet, 20,21 \bullet, 22 \bullet\end{array}$ \\
\hline & & & & & LE & PA & External & $\begin{array}{l}\text { *Stop Bleeding, Wound } \\
\text { Healing }\end{array}$ & \\
\hline & & Artemisia & Chaow & $\mathrm{H}$ & RT & EX & Internal & *Regulation of & $, 2 \bullet, 3 \bullet, 4 \bullet, 5 \bullet, 6 \bullet$, \\
\hline
\end{tabular}


Table 2 Medicinal uses of the reported taxa and their comparison with previous reports (Continued)

\begin{tabular}{|c|c|c|c|c|c|c|c|c|}
\hline \multirow[t]{2}{*}{ Family } & \multicolumn{2}{|l|}{ Nomenclature } & \multirow[t]{2}{*}{ Habit } & \multicolumn{4}{|c|}{ Medicinal uses } & \multirow[t]{2}{*}{ Previous reports } \\
\hline & Scientific name & Local name & & $\begin{array}{l}\text { Part } \\
\text { used }\end{array}$ & Preparation & Application & Disease treated & \\
\hline & \multirow{2}{*}{$\begin{array}{l}\text { vulgaris L. /AF- } \\
55\end{array}$} & & & & & & menstrual cycle & \multirow{2}{*}{$\begin{array}{l}7,8 \bullet, 9 \bullet, 10,11 \bullet, \\
12 \bullet, 13 \bullet, 14,15 \bullet, 16 \bullet \\
17 \bullet, 18 \bullet, 19 \bullet, 20 \bullet, 21 \bullet \\
22 \bullet\end{array}$} \\
\hline & & & & WP & IN & Internal & ${ }^{*}$ Cardiac problems & \\
\hline & \multirow{2}{*}{$\begin{array}{l}\text { Bidens biternata } \\
\text { (Lour.) Merr. \& } \\
\text { Sherff. /AF-79 }\end{array}$} & \multirow{2}{*}{$\begin{array}{l}\text { Suryaly/ } \\
\text { Palouthi }\end{array}$} & \multirow[t]{2}{*}{$\mathrm{H}$} & LE & JU & Internal & Sore infection & \multirow{2}{*}{$\begin{array}{l}1 \bullet, 2 \bullet, 3 \bullet, 4 \bullet, 5 \bullet, 6 \bullet, 7 \bullet \\
8 \bullet, 9 \bullet, 10 \bullet, 11 \bullet, 12 \bullet, 13 \bullet \\
14 \bullet, 15 \bullet, 16 \bullet, 17 \bullet, 18 \bullet, \\
19 \bullet, 20 \bullet, 21 \bullet, 22 \bullet\end{array}$} \\
\hline & & & & RT & PA & External & Toothache & \\
\hline & \multirow{4}{*}{$\begin{array}{l}\text { Carpesium } \\
\text { cernuum L. /AF- } \\
43\end{array}$} & \multirow[t]{4}{*}{ Marchi } & \multirow[t]{4}{*}{$\mathrm{H}$} & WP & EX & Internal & Cold, Fever & \multirow{4}{*}{$\begin{array}{l}1 \bullet, 2 \bullet, 3 \bullet, 4 \bullet, 5 \bullet, 6 \bullet, 7 \bullet \\
8 \bullet, 9 \bullet, 10 \bullet, 11 \bullet, 12 \bullet, \\
13 \bullet, 14 \bullet, 15 \bullet, 16 \bullet, 17 \bullet, \\
18 \bullet, 19 \bullet, 20 \bullet, 21 \bullet, 22 \bullet\end{array}$} \\
\hline & & & & & JU & Internal & Sore throat & \\
\hline & & & & RT & EX & Internal & Antibacterial & \\
\hline & & & & SD & $\mathrm{DE}$ & Internal & $\begin{array}{l}\text { Intestinal parasites, } \\
\text { Abdominal pain }\end{array}$ & \\
\hline & \multirow{4}{*}{$\begin{array}{l}\text { Cichorium } \\
\text { intybus L. /AF-2 }\end{array}$} & \multirow[t]{4}{*}{ Kasni } & \multirow[t]{4}{*}{$\mathrm{H}$} & RT & IN & Internal & Fever & \multirow{4}{*}{$\begin{array}{l}1 \bullet, 2 \bullet, 3,4 \bullet, 5 \bullet, 6 \bullet, \\
7,8 \bullet, 9,10 \bullet, 11 \\
12 \bullet, 13,14 \bullet, 15 \bullet, 16 \\
17 \bullet, 18 \bullet, 19 \bullet, 20 \bullet, 21 \bullet, \\
22 \bullet\end{array}$} \\
\hline & & & & LE & $\mathrm{DE}$ & Internal & $\begin{array}{l}\text { Indigestion, *Typhoid, } \\
\text { *Jaundice }\end{array}$ & \\
\hline & & & & & PD & Internal & ${ }^{*}$ Gout & \\
\hline & & & & LE & JU & Internal & $\begin{array}{l}{ }^{*} \text { Gall Stones, } \\
{ }^{*} \text { Gastrointestinal } \\
\text { problems }\end{array}$ & \\
\hline & \multirow{3}{*}{$\begin{array}{l}\text { Cirsium vulgare } \\
\text { (Savi) Ten. /AF- } \\
127\end{array}$} & \multirow[t]{3}{*}{ Kandayara } & \multirow[t]{3}{*}{$\mathrm{H}$} & WP & IN & External & Joint disorders & \multirow{3}{*}{$\begin{array}{l}1 \bullet, 2 \bullet, 3 \bullet, 4 \bullet, 5 \bullet, 6 \bullet, 7 \bullet, \\
8 \bullet, 9 \bullet, 10 \bullet, 11 \bullet, 12 \bullet, \\
13 \bullet, 14 \bullet, 15 \bullet, 16 \bullet, 17 \bullet, \\
18 \bullet, 19 \bullet, 20 \bullet, 21 \bullet, 21 \bullet, \\
22 \bullet\end{array}$} \\
\hline & & & & & DE & Internal & Piles & \\
\hline & & & & RT & $\mathrm{PO}$ & External & Sore Jaws & \\
\hline & \multirow{4}{*}{$\begin{array}{l}\text { Conyza } \\
\text { canadensis (L.) } \\
\text { Cronquist./AF- } \\
129\end{array}$} & \multirow[t]{4}{*}{ Kali Buti } & \multirow[t]{4}{*}{$\mathrm{H}$} & WP & EX & Internal & Diuretic, ${ }^{*}$ Cooling effect & \multirow{4}{*}{$\begin{array}{l}1 \bullet, 2 \bullet, 3 \bullet, 4 \bullet, 5 \bullet, 6 \bullet, 7 \\
8 \quad, 9 \bullet, 10 \bullet, 11 \bullet, 12 \bullet, 13 \bullet, \\
14,15 \bullet, 16,17 \bullet, 18 \bullet, \\
19 \bullet, 20 \bullet, 21 \bullet, 22 \bullet\end{array}$} \\
\hline & & & & & IN & Internal & $\begin{array}{l}\text { *Sore throat, *Diarrhea, } \\
\text { *nose bleeding }\end{array}$ & \\
\hline & & & & RT & $\mathrm{DE}$ & Internal & *Menstrual irregularities & \\
\hline & & & & LE & EX (Oil) & Internal & *Tonsils & \\
\hline & \multirow[t]{4}{*}{$\begin{array}{l}\text { Galinsoga } \\
\text { parviflora Cav./ } \\
\text { AF-73 }\end{array}$} & Peelibooti & $\mathrm{H}$ & WP & EX & External & $\begin{array}{l}\text { *Skin disease, } \\
\text { *Earache, *Scorpion } \\
\text { bites }\end{array}$ & $\begin{array}{l}1 \bullet, 2 \bullet, 3 \bullet, 4 \bullet, 5 \bullet, 6 \bullet, 7 \bullet \\
8 \bullet, 9 \bullet, 10 \bullet, 11 \bullet, 12 \\
13 \bullet, 14 \bullet, 15 \bullet, 16 \bullet, 17 \bullet, 18 \bullet,\end{array}$ \\
\hline & & & & $L F$ & $\mathrm{RB}$ & External & *Skin inflammation & \\
\hline & & & & & JU & External & *Blood clotting & \\
\hline & & & & & & Internal & ${ }^{*}$ Dysentery & \\
\hline & Gerbera & Bhurjali/ & $\mathrm{H}$ & LF & PA & External & Wounds, Skin Disease & $1 \bullet, 2 \bullet, 3 \bullet, 4 \quad, 5 \bullet, 6 \bullet, 7 \bullet$ \\
\hline & $\begin{array}{l}\text { gossypina } \\
\text { (Royle) } \\
\text { Beauverd./AF-27 }\end{array}$ & Ladrun & & AP & TE & Internal & *Nerve disorders & $\begin{array}{l}8 \bullet, 9 \bullet, 10 \bullet, 11 \bullet, 12 \bullet, 13 \bullet \\
14 \bullet, 15 \bullet, 16 \bullet, 17 \bullet, 18 \bullet \\
19 \bullet, 20 \bullet, 21 \bullet, 22 \bullet\end{array}$ \\
\hline & Inula spp. L./AF- & Peeli Boti & $\mathrm{H}$ & WP & EX & Internal & Diabetes, Fever & $1 \bullet, 2 \bullet, 3 \bullet, 4 \bullet, 5 \bullet, 6 \bullet, 7 \bullet$ \\
\hline & & & & RT & $\mathrm{DE}$ & Internal & $\begin{array}{l}\text { Digestive system } \\
\text { disorders, Asthma }\end{array}$ & $\begin{array}{l}8,9 \bullet, 10 \bullet, 11 \bullet, 12 \bullet, 13 \bullet \\
14 \bullet, 15 \bullet, 16 \bullet, 17 \bullet, 18 \bullet \\
19 \bullet, 20 \bullet, 21 \bullet, 22 \bullet\end{array}$ \\
\hline & Matricaria & Pineapple- & $\mathrm{H}$ & WP & EX & Internal & Vermifuge & $1 \bullet, 2 \bullet, 3 \bullet, 4 \bullet, 5 \bullet, 6 \bullet, 7 \bullet$ \\
\hline & $\begin{array}{l}\text { matricarioides } \\
\text { (Less.) Porter ex }\end{array}$ & & & & TE & Internal & Cold, Fever & $\begin{array}{l}8 \bullet, 9 \bullet, 10 \bullet, 11 \bullet, 12 \bullet \\
13 \bullet, 14 \bullet, 15 \bullet, 16 \bullet, 17 \bullet\end{array}$ \\
\hline & Britton./AF-46 & & & LF & IN & Internal & Stomach pain & $18 \bullet, 19 \bullet, 20 \bullet, 21 \bullet, 22 \bullet$ \\
\hline & & & & SD & $\mathrm{DE}$ & Internal & Indigestion & \\
\hline & $\begin{array}{l}\text { Myriactis } \\
\text { wallichii Less./ } \\
\text { AF-65 }\end{array}$ & $\begin{array}{l}\text { Safeed } \\
\text { surajmukhi }\end{array}$ & $\mathrm{H}$ & $L F$ & PA & External & Wound healing & $\begin{array}{l}1 \bullet, 2 \bullet, 3 \bullet, 4 \bullet, 5 \bullet, 6 \bullet, 7 \bullet, \\
8 \bullet, 9 \bullet, 10 \bullet, 11 \bullet, 12 \bullet, \\
13 \bullet, 14 \bullet, 15 \bullet, 16 \bullet, 17 \bullet, \\
18 \bullet, 19 \bullet, 20 \bullet, 21 \bullet, 22 \bullet\end{array}$ \\
\hline
\end{tabular}


Table 2 Medicinal uses of the reported taxa and their comparison with previous reports (Continued)

\begin{tabular}{|c|c|c|c|c|c|c|c|c|c|}
\hline \multirow{2}{*}{$\begin{array}{l}\text { Sr } \\
\#\end{array}$} & \multirow[t]{2}{*}{ Family } & \multicolumn{2}{|l|}{ Nomenclature } & \multirow[t]{2}{*}{ Habit } & \multicolumn{4}{|c|}{ Medicinal uses } & \multirow[t]{2}{*}{ Previous reports } \\
\hline & & Scientific name & Local name & & $\begin{array}{l}\text { Part } \\
\text { used }\end{array}$ & Preparation & Application & Disease treated & \\
\hline & & \multirow{4}{*}{$\begin{array}{l}\text { Parthenium } \\
\text { hysterophorus } \\
\text { L./AF-69 }\end{array}$} & \multirow[t]{4}{*}{ Gandibooti } & \multirow[t]{4}{*}{$\mathrm{H}$} & LF & JU & Internal & *Fever, Constipation & \multirow{4}{*}{$\begin{array}{l}1 \bullet, 2 \bullet, 3,4,5 \bullet, 6 \bullet, \\
7 \bullet, 8 \bullet, 9 \bullet, 10 \bullet, 11 \bullet, 12 \bullet, \\
13 \bullet, 14 \bullet, 15 \bullet, 16 \bullet, 17 \bullet, \\
18 \bullet, 19,20 \bullet, 21 \quad, 22 \bullet\end{array}$} \\
\hline & & & & & & $\mathrm{CH}$ & External & Toothache & \\
\hline & & & & & $\mathrm{FL}$ & PD & Internal & Diabetes & \\
\hline & & & & & WP & DE & Internal & Dysentery, ${ }^{*}$ Flue & \\
\hline & & \multirow{3}{*}{$\begin{array}{l}\text { Phagnalon } \\
\text { rupestre DC./AF- } \\
51\end{array}$} & \multirow[t]{3}{*}{ Jijjo Booti } & \multirow[t]{3}{*}{$\begin{array}{l}\text { Sub- } \\
\text { S }\end{array}$} & WP & $\mathrm{DE}$ & Internal & $\begin{array}{l}\text { Knee pain, Renal } \\
\text { stones }\end{array}$ & \multirow{3}{*}{$\begin{array}{l}1 \bullet, 2 \bullet, 3 \bullet, 4 \bullet, 5 \bullet, 6 \bullet, 7 \bullet, \\
8 \bullet, 9 \bullet, 10 \bullet, 11 \bullet, 12 \bullet, 13 \bullet, \\
14 \bullet, 15 \bullet, 16 \bullet, 17 \bullet, 18 \bullet, \\
19 \bullet, 20 \bullet, 21 \bullet, 22 \bullet\end{array}$} \\
\hline & & & & & $\mathrm{FP}$ & $\mathrm{HB}$ & Internal & Abdominal pain & \\
\hline & & & & & LF & PD & External & Joints pain & \\
\hline & & $\begin{array}{l}\text { Prenanthes } \\
\text { brunoniana } \\
\text { Wallex DC./AF- } \\
128\end{array}$ & $\begin{array}{l}\text { Himalayan } \\
\text { Blue Sow- } \\
\text { Thistle }\end{array}$ & $\mathrm{H}$ & WP & $\mathrm{PO}$ & External & Wounds, Sores & $\begin{array}{l}1 \bullet, 2 \bullet, 3 \bullet, 4 \bullet, 5 \bullet, 6 \bullet, 7 \bullet, \\
8 \bullet, 9 \bullet, 10 \bullet, 11 \bullet, 12 \bullet, 13 \bullet, \\
14 \bullet, 15 \bullet, 16 \bullet, 17 \bullet, 18 \bullet, \\
19 \bullet, 20 \bullet, 21 \bullet, 22 \bullet\end{array}$ \\
\hline & & \multirow{5}{*}{$\begin{array}{l}\text { Sigesbeckia } \\
\text { orientalis L./AF- } \\
97\end{array}$} & \multirow{5}{*}{$\begin{array}{l}\text { Yellow crown- } \\
\text { head }\end{array}$} & \multirow[t]{5}{*}{ C } & LF & EX & External & Rheumatism, Paralysis & \multirow{5}{*}{$\begin{array}{l}1 \bullet, 2 \bullet, 3 \bullet, 4 \bullet, 5 \bullet, 6 \bullet, 7 \bullet, \\
8 \bullet, 9 \bullet, 10 \bullet, 11 \bullet, 12 \bullet, 13 \bullet, \\
14 \bullet, 15 \bullet, 16 \bullet, 17 \bullet, 18 \bullet, \\
19 \bullet, 20 \bullet, 21 \bullet, 22 \bullet\end{array}$} \\
\hline & & & & & & PA & External & Wounds & \\
\hline & & & & & AP & $\mathrm{DE}$ & Internal & Hypertension & \\
\hline & & & & & & EX & External & Gout & \\
\hline & & & & & WP & EX & External & Sore between toes & \\
\hline & & \multirow{4}{*}{$\begin{array}{l}\text { Sonchus arvensis } \\
\text { L./AF- } 56\end{array}$} & \multirow{4}{*}{$\begin{array}{l}\text { Dodhak } \\
\text { Dodhal }\end{array}$} & \multirow[t]{4}{*}{$\mathrm{H}$} & $L F$ & $\mathrm{PO}$ & External & *Anti inflammation & \multirow{4}{*}{$\begin{array}{l}1 \bullet, 2 \bullet, 3 \bullet, 4 \bullet, 5 \bullet, 6 \bullet, 7 \bullet, \\
8 \bullet, 9 \bullet, 10 \bullet, 11 \bullet, 12 \bullet, \\
13 \bullet, 14 \bullet, 15 \bullet, 16 \bullet, 17 \bullet, \\
18 \bullet, 19,20 \bullet, 21 \bullet, 22 \bullet\end{array}$} \\
\hline & & & & & WP & PA & External & *Wounds cleaning & \\
\hline & & & & & & JU & Internal & ${ }^{*}$ Chronic fever & \\
\hline & & & & & RT & $\mathrm{DE}$ & Internal & Asthma & \\
\hline & & \multirow{5}{*}{$\begin{array}{l}\text { Sonchus } \\
\text { oleracus L./AF- } \\
106\end{array}$} & \multirow[t]{5}{*}{$\begin{array}{l}\text { Dodhak/ } \\
\text { Dodhal }\end{array}$} & \multirow[t]{5}{*}{$\mathrm{H}$} & $L F$ & $\mathrm{DE}$ & Internal & $\begin{array}{l}{ }^{*} \text { Constipation, }{ }^{*} \text { Body } \\
\text { weakness }\end{array}$ & $\begin{array}{l}1 \bullet, 2 \bullet, 3 \bullet, 4 \bullet, 5,6 \bullet, 7 \bullet, \\
8 \bullet, 9 \bullet, 10 \bullet, 11 \bullet, 12 \bullet, 13 \bullet,\end{array}$ \\
\hline & & & & & & $\mathrm{PO}$ & External & Swelling & $\begin{array}{l}14 \bullet, 15 \bullet, 16 \bullet, 17 \bullet, 18 \bullet \\
19 \bullet, 20 \bullet, 21 \bullet, 22 \bullet\end{array}$ \\
\hline & & & & & WP & JU & Internal & *Ulcers & \\
\hline & & & & & & IN & Internal & Diarrhea & \\
\hline & & & & & ST & $L X$ & External & Warts & \\
\hline & & Tagetes minuta & Setbergha & $\mathrm{H}$ & $\mathrm{FL}$ & EX & Internal & *Fever & $1 \bullet, 2 \bullet, 3 \bullet, 4 \bullet, 5 \quad, 6 \bullet$, \\
\hline & & L./AF-139 & & & LF & JU & Internal & *Piles & $\begin{array}{l}7,8 \bullet, 9,10 \bullet, 11 \bullet, \\
12,13 \bullet, 14 \bullet, 15 \bullet, 16 \bullet\end{array}$ \\
\hline & & & & & & & External & ${ }^{*}$ Earache, ${ }^{*}$ Ophthalmic & $\begin{array}{l}17 \bullet, 18 \bullet, 19 \bullet, 20 \bullet, 21 \bullet \\
22 \bullet\end{array}$ \\
\hline & & Taraxacum & Hand & $\mathrm{H}$ & LF & VG & Internal & *Diabetes & $1,2 \bullet, 3,4,5$, \\
\hline & & $\begin{array}{l}\text { Officinale F.H. } \\
\text { Wigg./AF-121 }\end{array}$ & & & & $L X$ & Internal & $\begin{array}{l}\text { *To stimulate } \\
\text { Gallbladder, Indigestion }\end{array}$ & $\begin{array}{l}6,18,9,10 \bullet, 11 \bullet, \\
12 \bullet, 13 \bullet, 14 \bullet, 15 \bullet, 16 \\
17 \bullet, 18 \bullet, 19 \bullet, 20,21 \bullet,\end{array}$ \\
\hline & & & & & WP & JU & Internal & Liver disease, Jaundice & 22 \\
\hline & & & & & $\mathrm{RH}$ & $\mathrm{DE}$ & Internal & Jaundice & \\
\hline 8 & Balsaminaceae & $\begin{array}{l}\text { Impatiens } \\
\text { edgeworthii }\end{array}$ & Tilchawli & $\mathrm{H}$ & WP & EX & Internal & $\begin{array}{l}\text { *Urinary tract } \\
\text { infection }\end{array}$ & $\begin{array}{l}1 \bullet, 2 \bullet, 3 \bullet, 4 \bullet, 5 \bullet, 6 \\
7 \bullet, 8 \bullet, 9 \bullet, 10 \bullet, 11 \bullet, 12 \bullet,\end{array}$ \\
\hline & & HOOK. †./AF-IOS & & & & & External & *Burns & $18 \bullet, 19 \bullet, 20,21 \bullet, 22 \bullet$ \\
\hline & & & & & & & Internal & ${ }^{*}$ Fever & \\
\hline & & $\begin{array}{l}\text { Impatiens } \\
\text { glandulifera }\end{array}$ & Tilcawli & $\mathrm{H}$ & RT & PA & External & $\begin{array}{l}{ }^{*} \text { Cooling effect on } \\
\text { hands and Foot }\end{array}$ & $\begin{array}{l}1,2 \bullet, 3 \bullet, 4 \bullet, 5 \bullet, 6 \bullet, 7 \bullet, \\
8 \bullet, 9 \bullet, 10 \bullet, 11 \bullet, 12 \bullet, 13 \bullet,\end{array}$ \\
\hline & & Royle./AF-82 & & & LF & $\mathrm{DE}$ & Internal & Mental tension & $\begin{array}{l}14 \bullet, 15 \bullet, 16 \bullet, 1 / \bullet, 18 \bullet \\
19 \bullet, 20 \bullet, 21 \bullet, 22 \bullet\end{array}$ \\
\hline & & & & & $\mathrm{FL}$ & TE & External & *Eye wash & \\
\hline 9 & Berberidaceae & $\begin{array}{l}\text { Berberis lycium } \\
\text { Royle./AF-4 }\end{array}$ & Sumbal & S & LE & PA & External & $\begin{array}{l}\text { *Bleeding, Wound } \\
\text { healing }\end{array}$ & $\begin{array}{ll}1 & , 2 \bullet, 3,4,5 \\
6 & , 7 \bullet, 8,9,10\end{array}$ \\
\hline
\end{tabular}


Table 2 Medicinal uses of the reported taxa and their comparison with previous reports (Continued)

\begin{tabular}{|c|c|c|c|c|c|c|c|c|c|}
\hline \multirow{2}{*}{$\begin{array}{l}\mathrm{Sr} \\
\#\end{array}$} & \multirow[t]{2}{*}{ Family } & \multicolumn{2}{|l|}{ Nomenclature } & \multirow[t]{2}{*}{ Habit } & \multicolumn{4}{|c|}{ Medicinal uses } & \multirow[t]{2}{*}{ Previous reports } \\
\hline & & Scientific name & Local name & & $\begin{array}{l}\text { Part } \\
\text { used }\end{array}$ & Preparation & Application & Disease treated & \\
\hline \multirow{5}{*}{10} & & & & & RT & EX & Internal & *Joint Problems & $11,12,13,14$ \\
\hline & & & & & BA & PD & Internal & Bleeding gums & $20 \bullet, 21 \bullet, 22$ \\
\hline & \multirow[t]{3}{*}{ Boraginaceae } & \multirow{3}{*}{$\begin{array}{l}\text { Cynoglossum } \\
\text { lanceolatum } \\
\text { Forssk./AF-23 }\end{array}$} & \multirow[t]{3}{*}{ Churuun } & \multirow[t]{3}{*}{$\mathrm{H}$} & RT & EX & Internal & *Throat diseases & \multirow{3}{*}{$\begin{array}{l}1,2 \bullet, 3 \bullet, 4 \bullet, 5 \bullet, 6 \bullet, \\
7 \bullet, 8,9 \bullet, 10 \bullet, 11 \bullet, 12 \bullet, \\
13 \bullet, 14 \bullet, 15 \bullet, 16 \bullet, 17 \bullet, \\
18 \bullet, 19 \bullet, 20,21 \bullet, 22 \bullet\end{array}$} \\
\hline & & & & & $F R$ & $\mathrm{CH}$ & External & *Toothache & \\
\hline & & & & & LE & PD & Internal & $\begin{array}{l}\text { *Kidney disorder, } \\
\text { *Tooth and gum } \\
\text { diseases }\end{array}$ & \\
\hline \multirow[t]{3}{*}{11} & \multirow[t]{3}{*}{ Brassicaceae } & \multirow{3}{*}{$\begin{array}{l}\text { Capsella bursa- } \\
\text { pastoris (L.) } \\
\text { Medick./AF-94 }\end{array}$} & \multirow[t]{3}{*}{ Doddipatti } & \multirow[t]{3}{*}{$\mathrm{H}$} & AP & VG & Internal & Diarrhea & \multirow{3}{*}{$\begin{array}{l}1 \bullet, 2 \bullet, 3 \bullet, 4 \bullet, 5 \bullet, 6 \bullet, 7 \bullet, \\
8 \bullet, 9 \bullet, 10 \bullet, 11 \bullet, 12 \bullet, 13 \bullet \\
14 \bullet, 15 \bullet, 16 \bullet, 17 \bullet, 18 \bullet, \\
19,20 \bullet, 21 \bullet, 22 \bullet\end{array}$} \\
\hline & & & & & LE & $\mathrm{DE}$ & Internal & Menstrual disorders & \\
\hline & & & & & WP & $J U$ & Internal & * Nose bleeding & \\
\hline \multirow[t]{3}{*}{12} & \multirow[t]{3}{*}{ Buxaceae } & \multirow{3}{*}{$\begin{array}{l}\text { Sarcococca } \\
\text { saligna (D. Don) } \\
\text { Müll. Arg./AF-64 }\end{array}$} & \multirow[t]{3}{*}{ Niaroi/Ndroon } & \multirow[t]{3}{*}{ S } & $\mathrm{SH}$ & EX & External & Joint pain & \multirow{3}{*}{$\begin{array}{l}1,2 \bullet, 3 \bullet, 4 \bullet, 5 \bullet, 6, \\
7,8 \bullet, 9 \bullet, 10 \bullet, 11 \bullet, \\
12 \bullet, 13 \bullet, 14 \bullet, 15 \bullet, 16 \bullet, \\
17 \bullet, 18 \bullet, 19 \bullet, 20 \bullet, 21 \bullet, \\
22 \bullet\end{array}$} \\
\hline & & & & & RT & JU & Internal & Gonorrhea & \\
\hline & & & & & LF & IN & Internal & Blood purification & \\
\hline 13 & Campanulaceae & $\begin{array}{l}\text { Campanula } \\
\text { pallida Wall./AF- } \\
111\end{array}$ & Beli Phool & $\mathrm{H}$ & WP & EX & Internal & $\begin{array}{l}\text { Dysentery, Liver } \\
\text { disorders }\end{array}$ & $\begin{array}{l}1 \bullet, 2 \bullet, 3 \bullet, 4 \bullet, 5 \bullet, 6 \bullet, 7 \bullet, \\
8 \bullet, 9 \bullet, 10 \bullet, 11 \bullet, 12 \bullet \\
13 \bullet, 14 \bullet, 15 \bullet, 16 \bullet, 17 \bullet, \\
18 \bullet, 19 \bullet, 20 \bullet, 21 \bullet, 22 \bullet\end{array}$ \\
\hline \multirow[t]{2}{*}{14} & \multirow[t]{2}{*}{ Cannabaceae } & \multirow{2}{*}{$\begin{array}{l}\text { Cannabis sativa } \\
\text { L./AF-83 }\end{array}$} & \multirow[t]{2}{*}{ Kamm/Bhang } & \multirow[t]{2}{*}{$\mathrm{H}$} & LE & $\mathrm{TE}$ & Internal & *Joint problems & $1 \bullet, 2,3 \bullet, 4 \bullet, 5 \bullet, 6 \bullet$ \\
\hline & & & & & WP & $\mathrm{DE}$ & Internal & Whooping cough & $\begin{array}{l}12,8,13,14,15 \bullet \\
16,17,18,19 \\
20 \bullet, 21,22 \bullet\end{array}$ \\
\hline 15 & Convolvulaceae & Convolvulus & Speaker Booti & C & WP & VG & Internal & Skin Diseases & $1 \bullet, 2,3 \bullet, 4 \bullet, 5,6 \bullet$ \\
\hline & & arvensis L./AF-30 & & & RT & EX & External & Dandruff & $\begin{array}{l}7,, 8 \bullet, 9 \bullet, 10 \bullet, 11 \bullet, \\
12 \bullet, 13,14 \bullet, 15 \bullet, 16 \bullet, \\
17 \bullet, 18 \bullet, 19 \bullet, 20 \bullet, 21 \\
22 \bullet\end{array}$ \\
\hline & & $\begin{array}{l}\text { Ipomoea } \\
\text { purpurea (L.) }\end{array}$ & Eieer & C & SD & PD & Internal & $\begin{array}{l}\text { Mental disorders, } \\
\text { Constipation, Diuretic }\end{array}$ & $\begin{array}{l}1 \bullet, 2 \bullet, 3 \bullet, 4 \bullet, 5 \bullet, 6 \bullet, 7 \bullet, \\
8 \bullet, 9 \bullet, 10 \bullet, 11 \bullet, 12 \bullet, 13 \bullet,\end{array}$ \\
\hline & & Rotn./AF-/O & & & RT & EX & Internal & Syphilis & $19 \bullet, 20 \bullet, 21 \bullet, 22 \bullet$ \\
\hline & & & & & $\mathrm{FL}$ & EX & Internal & Laxative, Purgative & \\
\hline 16 & Cyperaceae & $\begin{array}{l}\text { Cyperus } \\
\text { serotinus Rottb./ } \\
\text { AF-116 }\end{array}$ & Deela Ghass & $\mathrm{H}$ & RT & EX & Internal & Tonic, Stimulant & $\begin{array}{l}1 \bullet, 2 \bullet, 3 \bullet, 4 \bullet, 5 \bullet, 6 \bullet, 7 \bullet, \\
8 \bullet, 9 \bullet, 10 \bullet, 11 \bullet, 12 \bullet, \\
13 \bullet, 14 \bullet, 15 \bullet, 16 \bullet, 17 \bullet, 18 \bullet \\
19 \bullet, 20 \bullet, 21 \bullet, 22 \bullet\end{array}$ \\
\hline & & $\begin{array}{l}\text { Eriophorum } \\
\text { comosum (Wall.) } \\
\text { Nees. /AF-90 }\end{array}$ & Berbaya & $\mathrm{H}$ & WP & PD & Internal & $\begin{array}{l}\text { Abdominal pain, } \\
\text { Kidney pain }\end{array}$ & $\begin{array}{l}1 \bullet, 2 \bullet, 3 \bullet, 4 \bullet, 5 \bullet, 6 \bullet, 7 \bullet, \\
8 \bullet, 9 \bullet, 10 \bullet, 11 \bullet, 12 \bullet, 13 \bullet, \\
14 \bullet, 15 \bullet, 16 \bullet, 17 \bullet, 18 \bullet, \\
19 \bullet, 20 \bullet, 21 \bullet, 22 \bullet\end{array}$ \\
\hline 17 & Dryopteridaceae & Dryopteris filix- & Kungi & $\mathrm{H}$ & FD & VG & Internal & Diabetes & $1 \bullet, 2 \bullet, 3 \bullet, 4 \bullet, 5 \bullet, 6 \bullet, 7 \bullet$, \\
\hline & & $\begin{array}{l}\text { mas (L.) Schott. } \\
\text { /AF-17 }\end{array}$ & & & RT & EX & Internal & To treat Tapeworms & $\begin{array}{l}8 \bullet, 9 \bullet, 10 \bullet, 1 \\
14 \bullet, 15 \bullet, 16 \bullet, 17 \bullet, 18 \bullet,\end{array}$ \\
\hline & & & & & & & External & $\begin{array}{l}\text { Muscle pain, Paralysis, } \\
\text { Sciatica }\end{array}$ & $19 \bullet, 20 \bullet, 21 \bullet, 22 \bullet$ \\
\hline 18 & Ebenaceae & $\begin{array}{l}\text { Diospyros lotus } \\
\text { L. /AF-119 }\end{array}$ & Amlook & T & $F R$ & ET & Internal & $\begin{array}{l}\text { *Stomach disease, } \\
\text { *Fever }\end{array}$ & $\begin{array}{l}1 \bullet, 2 \bullet, 3 \bullet, 4 \bullet, 5 \bullet, 6 \bullet, 7 \\
8 \quad, 9 \bullet, 10,11 \bullet, 12 \bullet\end{array}$ \\
\hline & & & & & TW & $\mathrm{RB}$ & External & $\begin{array}{l}\text { *Toothache, *Gums and } \\
\text { lips coloring }\end{array}$ & $\begin{array}{l}13 \\
18 \bullet, 19 \bullet, 20 \bullet, 21 \bullet, 22 \bullet\end{array}$ \\
\hline 19 & Elaeagnaceae & $\begin{array}{l}\text { Elaeagnus } \\
\text { umbellata }\end{array}$ & Kankoli & S & SD & EX (Oil) & Internal & $\begin{array}{l}\text { Breathing disorders, } \\
\text { Lungs disease }\end{array}$ & $\begin{array}{l}1 \bullet, 2 \bullet, 3,4,5 \bullet, 6 \bullet, \\
7 \bullet, 8 \bullet, 9 \bullet, 10 \bullet, 11 \bullet, 12 \bullet,\end{array}$ \\
\hline & & & & & TW & $\mathrm{RB}$ & External & Toothache & $\begin{array}{l}13 \bullet, 14 \bullet, 15 \bullet, 16 \bullet, 17 \bullet \\
18 \bullet, 19 \bullet, 20 \bullet, 21 \bullet, 22 \bullet\end{array}$ \\
\hline
\end{tabular}


Table 2 Medicinal uses of the reported taxa and their comparison with previous reports (Continued)

\begin{tabular}{|c|c|c|c|c|c|c|c|c|c|}
\hline \multirow{2}{*}{$\begin{array}{l}\text { Sr } \\
\#\end{array}$} & \multirow[t]{2}{*}{ Family } & \multicolumn{2}{|l|}{ Nomenclature } & \multirow[t]{2}{*}{ Habit } & \multicolumn{4}{|c|}{ Medicinal uses } & \multirow[t]{2}{*}{ Previous reports } \\
\hline & & Scientific name & Local name & & $\begin{array}{l}\text { Part } \\
\text { used }\end{array}$ & Preparation & Application & Disease treated & \\
\hline & & & & & $F R$ & ET & Internal & ${ }^{*}$ Mouth sore & \\
\hline \multirow[t]{11}{*}{20} & \multirow[t]{11}{*}{ Euphorbiaceae } & \multirow{4}{*}{$\begin{array}{l}\text { Euphorbia indica } \\
\text { Lam. /AF-15 }\end{array}$} & \multirow{4}{*}{$\begin{array}{l}\text { Dodhale/ } \\
\text { Dodhal }\end{array}$} & \multirow[t]{4}{*}{$\mathrm{H}$} & \multirow[t]{4}{*}{ WP } & DE & Internal & Diarrhea, Dysentery & \multirow{4}{*}{$\begin{array}{l}1 \bullet, 2 \bullet, 3 \bullet, 4 \bullet, 5 \bullet, 6 \bullet, 7 \bullet, \\
8 \bullet, 9 \bullet, 10 \bullet, 11 \bullet, 12 \bullet, 13 \bullet, \\
14 \bullet, 15 \bullet, 16 \bullet, 17 \bullet, 18 \bullet, \\
19 \bullet, 20 \bullet, 21 \bullet, 22 \bullet\end{array}$} \\
\hline & & & & & & $L X$ & Internal & Purgative & \\
\hline & & & & & & & External & Eye infection & \\
\hline & & & & & & PD & External & Oedema & \\
\hline & & \multirow{3}{*}{$\begin{array}{l}\text { Euphorbia } \\
\text { prostrata Aiton. } \\
\text { /AF- } 49\end{array}$} & \multirow{3}{*}{$\begin{array}{l}\text { Dodhal/Hazar } \\
\text { Dani }\end{array}$} & \multirow[t]{3}{*}{$\mathrm{H}$} & WP & DE & Internal & Dysentery, Diarrhea & \multirow{3}{*}{$\begin{array}{l}1 \bullet, 2 \bullet, 3 \bullet, 4 \bullet, 5 \bullet, 6 \bullet, 7 \bullet, \\
8 \bullet, 9 \bullet, 10 \bullet, 11 \bullet, 12 \bullet, 13 \bullet, \\
14 \bullet, 15 \bullet, 16 \bullet, 17 \bullet, 18 \bullet, \\
19 \bullet, 20 \bullet, 21 \bullet, 22 \bullet\end{array}$} \\
\hline & & & & & AP & IN & Internal & *Stomachache & \\
\hline & & & & & LF & PD & External & *Headache & \\
\hline & & \multirow{4}{*}{$\begin{array}{l}\text { Ricinus } \\
\text { communis L./ } \\
\text { AF-57 }\end{array}$} & \multirow[t]{4}{*}{ Hernoli } & \multirow[t]{4}{*}{ S } & RT & EX & External & $\begin{array}{l}\text { *Muscles weakness, } \\
\text { *Gout disease }\end{array}$ & \multirow{4}{*}{$\begin{array}{l}1,2,3 \bullet, 4 \bullet, 5,6 \bullet, \\
7 \bullet, 8 \bullet, 9,10,11 \bullet, \\
12 \bullet, 13 \bullet, 14,15,16 \bullet, \\
18 \bullet, 19,20 \bullet, 21 \bullet, 22 \bullet\end{array}$} \\
\hline & & & & & LF & EX & Internal & $\begin{array}{l}\text { *To remove poisonous } \\
\text { from body }\end{array}$ & \\
\hline & & & & & \multirow[t]{2}{*}{ SD } & \multirow[t]{2}{*}{ EX (oil) } & Internal & *Scorpion bite & \\
\hline & & & & & & & External & *Eye Disease, Dandruff & \\
\hline \multirow[t]{18}{*}{21} & \multirow[t]{18}{*}{ Fabaceae } & \multirow{5}{*}{$\begin{array}{l}\text { Acacia nilotica } \\
\text { (L.) Willd. ex } \\
\text { Delile/AF-37 }\end{array}$} & Kikar & T & ST & Ash (PD) & External & ${ }^{*}$ Eye Diseases & $1 \bullet, 2,3 \bullet, 4 \bullet, 5,6 \bullet$ \\
\hline & & & & & BA & $\mathrm{DE}$ & External & Toothache & $\begin{array}{l}7 \bullet, 8,9 \bullet, 10 \bullet, 11 \bullet, \\
12 \bullet, 13 \bullet, 14,15 \bullet, 16 \bullet,\end{array}$ \\
\hline & & & & & $\mathrm{FL}$ & DE & External & *Earache & $17 \bullet, 18,19,20 \bullet$ \\
\hline & & & & & SD & PD & Internal & *Kidney pain, Diabetes & \\
\hline & & & & & & & External & Toothpowder & \\
\hline & & Desmodium & Mangkit & S & WP & Extract & Internal & *Diarrhea & $1 \bullet, 2 \bullet, 3 \bullet, 4 \bullet, 5 \bullet, 6 \bullet, 7 \bullet$, \\
\hline & & $\begin{array}{l}\text { elegans DC./AF- } \\
31\end{array}$ & parang & & LE & Extract & External & ${ }^{*}$ Eye Infection & $\begin{array}{l}8 \bullet, 9 \bullet, 10 \bullet, 11 \bullet, 12 \bullet, 13 \bullet \\
14,15 \bullet, 16 \bullet, 17 \bullet, 18 \bullet,\end{array}$ \\
\hline & & & & & & & Internal & $\begin{array}{l}{ }^{*} \text { Cough, }{ }^{*} \text { Fever, } \\
{ }^{*} \text { Vomiting }\end{array}$ & $19 \bullet, 20 \bullet, 21 \bullet, 22 \bullet$ \\
\hline & & & & & RT & Powder & External & $\begin{array}{l}\text { *Scorpion and Snake } \\
\text { bites }\end{array}$ & \\
\hline & & Indigofera & Jand & S & BR & PD & Internal & Whooping cough & $1 \bullet, 2 \bullet, 3 \bullet, 4 \bullet, 5 \bullet, 6 \bullet, 7 \bullet$ \\
\hline & & $\begin{array}{l}\text { Wall.ex Brandis./ } \\
\text { AF-33 }\end{array}$ & & & LF & EX & Internal & Mouth infection & $\begin{array}{l}13 \bullet, 14,15 \bullet, 16 \bullet, 17 \bullet \\
18 \bullet, 19 \bullet, 20 \quad, 21 \bullet, 22 \bullet\end{array}$ \\
\hline & & Lespedeza & Silky bush- & $\mathrm{H}$ & $\mathrm{SH}$ & DE & Internal & Dysentery, Diarrhea & $1 \bullet, 2 \bullet, 3 \bullet, 4 \bullet, 5 \bullet, 6 \bullet, 7 \bullet$, \\
\hline & & $\begin{array}{l}\text { Juncea (L. f.) } \\
\text { Pers./AF-133 }\end{array}$ & & & WP & $\mathrm{DE}$ & External & $\begin{array}{l}\text { *Skin ulcers, } \\
\text { *Toothache }\end{array}$ & $\begin{array}{l}8 \bullet, 9 \bullet, 10 \bullet, 11 \bullet, 12 \bullet, \\
13 \bullet, 14 \bullet, 15 \bullet, 16 \bullet, 17 \bullet, 18 \bullet, \\
19 \bullet, 20 \bullet, 21 \bullet, 22 \bullet\end{array}$ \\
\hline & & Medicago & Sirri & $\mathrm{H}$ & SD & PD & Internal & Indigestion & $1 \bullet, 2 \bullet, 3 \bullet, 4 \bullet, 5 \bullet, 6 \bullet, 7 \bullet$, \\
\hline & & $\begin{array}{l}\text { Iupulina L.JAF- } \\
132\end{array}$ & & & WP & EX & Internal & Antibacterial & $\begin{array}{l}14 \bullet, 15 \bullet, 16 \bullet, 17 \bullet, 18 \bullet, \\
19 \bullet, 20 \bullet, 21 \bullet, 22 \bullet\end{array}$ \\
\hline & & $\begin{array}{l}\text { Trifolium } \\
\text { pratense L./AF- } \\
42\end{array}$ & Trapetra & $\mathrm{H}$ & $\begin{array}{l}\mathrm{FL} \\
\text { and } \\
\mathrm{LF}\end{array}$ & EX & Internal & $\begin{array}{l}\text { Minimize menopause } \\
\text { symptoms }\end{array}$ & $\begin{array}{l}1 \bullet, 2 \bullet, 3 \bullet, 4 \bullet, 5 \bullet, 6 \bullet, 7 \bullet, \\
8 \bullet, 9 \bullet, 10 \bullet, 11 \bullet, 12 \bullet, \\
13 \bullet, 14 \bullet, 15 \bullet, 16,17 \bullet,\end{array}$ \\
\hline & & & & & WP & $\mathrm{PO}$ & External & *Breast cancer & \\
\hline & & & & & & DE & Internal & $\begin{array}{l}{ }^{*} \text { Cancer, }{ }^{*} \text { Whooping } \\
\text { Cough, }{ }^{*} \text { Gout disease }\end{array}$ & \\
\hline 22 & Fagaceae & Quercus incana & Rein & T & SD & PD & Internal & *Diuretic & $1,2 \bullet, 3,4,5 \bullet, 6 \bullet$ \\
\hline & & $\begin{array}{l}\text { W. Bartram./AF- } \\
32\end{array}$ & & & $G L$ & $\mathrm{DE}$ & Internal & $\begin{array}{l}\text { Joint swelling, } \\
\text { Dysentery }\end{array}$ & $\begin{array}{l}7,8 \bullet, 9 \bullet, 10 \bullet, 11,12 \bullet, \\
13 \bullet, 14 \bullet, 15,16 \bullet, 17 \bullet \\
18 \bullet, 19 \bullet, 20 \bullet, 21 \bullet, 22 \bullet\end{array}$ \\
\hline & & & & & STb & PD & External & *Skin ulcer & \\
\hline & & & & & & DE & Internal & Throat pain & \\
\hline 23 & Gentianaceae & Gentianodes & Neeli Booti & $\mathrm{H}$ & WP & $\mathrm{DE}$ & Internal & Jaundice, Cough & $1 \bullet, 2 \bullet, 3 \bullet, 4 \bullet, 5 \bullet, 6 \bullet, 7 \bullet$, \\
\hline
\end{tabular}


Table 2 Medicinal uses of the reported taxa and their comparison with previous reports (Continued)

\begin{tabular}{|c|c|c|c|c|c|c|c|c|c|}
\hline \multirow{2}{*}{$\begin{array}{l}\mathrm{Sr} \\
\#\end{array}$} & \multirow[t]{2}{*}{ Family } & \multicolumn{2}{|l|}{ Nomenclature } & \multirow[t]{2}{*}{ Habit } & \multicolumn{4}{|c|}{ Medicinal uses } & \multirow[t]{2}{*}{ Previous reports } \\
\hline & & Scientific name & Local name & & $\begin{array}{l}\text { Part } \\
\text { used }\end{array}$ & Preparation & Application & Disease treated & \\
\hline \multirow{10}{*}{24} & & $\begin{array}{l}\text { olivieri (Griseb.) } \\
\text { Omer, Ali \& } \\
\text { Qaiser./AF-44 }\end{array}$ & & & & PD & Internal & Throat problem & $\begin{array}{l}8 \bullet, 9 \bullet, 10 \bullet, 11 \bullet, 12 \bullet, 13 \bullet, \\
14 \bullet, 15 \bullet, 16 \bullet, 17 \bullet, 18 \bullet, \\
19 \bullet, 20 \bullet, 21 \bullet, 22 \bullet\end{array}$ \\
\hline & & $\begin{array}{l}\text { Swertia cordata } \\
\text { (Wall. ex G. } \\
\text { Don) C.B. }\end{array}$ & Plamas & $\mathrm{H}$ & WP & EX & Internal & $\begin{array}{l}\text { Pneumonia fever, } \\
\text { Throat problems, } \\
\text { Malarial fever }\end{array}$ & $\begin{array}{l}1 \bullet, 2 \bullet, 3 \bullet, 4 \bullet, 5 \bullet, 6 \bullet, 7 \bullet, \\
8 \bullet, 9 \bullet, 10 \bullet, 11 \bullet, 12 \bullet, \\
13 \bullet, 14 \bullet, 15 \bullet, 16 \bullet, 17 \bullet,\end{array}$ \\
\hline & & & & & & IN & Internal & To kill intestinal worms & \\
\hline & & & & & & PD & Internal & Tonic & \\
\hline & & $\begin{array}{l}\text { Swertia } \\
\text { paniculata Wall./ }\end{array}$ & $\begin{array}{l}\text { Plamas/Jabba } \\
\text { jarri }\end{array}$ & $\mathrm{H}$ & WP & EX & Internal & $\begin{array}{l}\text { *Malarial Fever, } \\
\text { *Diarrhea }\end{array}$ & $\begin{array}{l}1 \bullet, 2 \bullet, 3 \bullet, 4 \bullet, 5 \bullet, 6, \\
7 \bullet, 8 \bullet, 9 \bullet, 10 \bullet, 11 \bullet, 12 \bullet,\end{array}$ \\
\hline & & AF-50 & & & & DE & Internal & *Tonic & $\begin{array}{l}13 \bullet, 14 \bullet, 15 \bullet, 16 \bullet, 17 \bullet \\
18 \bullet, 19 \bullet, 20 \bullet, 21 \bullet, 22 \bullet\end{array}$ \\
\hline & \multirow[t]{4}{*}{4 Hypericaceae } & \multirow{4}{*}{$\begin{array}{l}\text { Hypericum } \\
\text { perforatum L./ } \\
\text { AF-59 }\end{array}$} & \multirow[t]{4}{*}{ Sharan Gulab } & \multirow[t]{4}{*}{$\mathrm{H}$} & $\mathrm{SH}$ & DE & Internal & *Anxiety & \multirow{4}{*}{$\begin{array}{l}1 \bullet, 2 \bullet, 3,4,5 \bullet, 6 \bullet \\
7 \bullet, 8 \bullet, 9 \bullet, 10 \bullet, 11 \bullet, 12 \bullet \\
13 \bullet, 14 \bullet, 15 \bullet, 16 \bullet, 17 \bullet \\
18 \bullet, 19 \bullet, 20 \bullet, 21 \bullet, 22 \bullet\end{array}$} \\
\hline & & & & & WP & EX & Internal & *Depression & \\
\hline & & & & & & & External & $\begin{array}{l}\text { *Bruises, Wounds, } \\
\text { *Intestinal problems }\end{array}$ & \\
\hline & & & & & $\mathrm{FL}$ & IN & External & Swelling, *Sunburns & \\
\hline \multirow[t]{23}{*}{25} & \multirow[t]{23}{*}{ Lamiaceae } & \multirow{2}{*}{$\begin{array}{l}\text { Ajuga bracteosa } \\
\text { Wall. ex Benth./ } \\
\text { AF-20 }\end{array}$} & \multirow[t]{2}{*}{$\begin{array}{l}\text { Thandi Jarri/ } \\
\text { Ratti Booti }\end{array}$} & \multirow[t]{2}{*}{$\mathrm{H}$} & LE & DE & Internal & $\begin{array}{l}\text { Skin Infection, Stomach } \\
\text { problem }\end{array}$ & \multirow{2}{*}{$\begin{array}{l}1,2,3,4,5 \bullet, 6 \bullet \\
7 \bullet, 8 \bullet, 9 \bullet, 10,11 \bullet, 12 \bullet, \\
13 \bullet, 14 \bullet, 15,16 \bullet, 17 \\
18 \bullet, 19 \bullet, 20 \bullet, 21 \bullet, 22 \bullet\end{array}$} \\
\hline & & & & & WP & EX & Internal & Jaundice, *Ulcer & \\
\hline & & \multirow{2}{*}{$\begin{array}{l}\text { Ajuga parviflora } \\
\text { Benth./AF-21 }\end{array}$} & \multirow[t]{2}{*}{ Thandi Jarri } & \multirow[t]{2}{*}{$\mathrm{H}$} & LE & EX & Internal & Gastric problem & \multirow{2}{*}{$\begin{array}{l}1 \bullet, 2 \bullet, 3 \bullet, 4 \bullet, 5 \bullet, 6 \bullet, 7 \bullet, \\
8 \bullet, 9 \bullet, 10 \bullet, 11 \bullet, 12 \bullet, 13 \bullet \\
14 \bullet, 15 \bullet, 16 \bullet, 17 \bullet, 18 \bullet, \\
19 \bullet, 20 \bullet, 21 \bullet, 22 \bullet\end{array}$} \\
\hline & & & & & WP & EX & Internal & $\begin{array}{l}\text { Hypertension, } \\
\text { Headache }\end{array}$ & \\
\hline & & \multirow{4}{*}{$\begin{array}{l}\text { Isodon rugosus } \\
\text { (Wall. ex Benth.) } \\
\text { Codd./AF-80 }\end{array}$} & \multirow[t]{4}{*}{ Chitta Manja } & \multirow[t]{4}{*}{ S } & SD & DE & Internal & Blood purifier & \multirow{4}{*}{$\begin{array}{l}1 \bullet, 2 \bullet, 3,4,5 \bullet, 6 \bullet, \\
7 \bullet, 8 \bullet, 9 \bullet, 10 \bullet, 11 \bullet, 12 \\
13 \bullet, 14,15 \bullet, 16 \bullet, 17 \bullet, \\
18 \bullet, 19 \bullet, 20 \bullet, 21 \bullet, 22 \bullet\end{array}$} \\
\hline & & & & & $\mathrm{SH}$ & EX & Internal & Abdominal pain & \\
\hline & & & & & $L F$ & PD & Internal & ${ }^{*}$ Digestive problem & \\
\hline & & & & & & PA & External & Blood clotting & \\
\hline & & \multirow[t]{2}{*}{$\begin{array}{l}\text { Mentha arvensis } \\
\text { L./AF-28 }\end{array}$} & Podina & $\mathrm{H}$ & $L F$ & DE & Internal & $\begin{array}{l}\text { Stomach acidity, } \\
\text { Indigestion, Vomiting }\end{array}$ & $\begin{array}{l}1 \bullet, 2,3 \bullet, 4 \bullet, 5 \bullet, 6 \bullet, 7 \bullet \\
8 \bullet, 9,10 \bullet, 11 \bullet, 12 \bullet, 13 \bullet,\end{array}$ \\
\hline & & & & & & EX & Internal & Dysentery, Diarrhea & $\begin{array}{l}14 \bullet, 15 \bullet, 16 \bullet, 17 \\
19 \bullet, 20 \bullet, 21 \bullet, 22 \bullet\end{array}$ \\
\hline & & $\begin{array}{l}\text { Mentha } \\
\text { longifolia (L.) }\end{array}$ & Bareena & $\mathrm{H}$ & LF & $\mathrm{DE}$ & Internal & $\begin{array}{l}\text { Digestive disorders, } \\
\text { Abdominal disorders }\end{array}$ & $\begin{array}{l}1 \quad, 2 \bullet, 3 \bullet, 4 \bullet, 5,6 \bullet, \\
7 \quad, 8 \bullet, 9,10 \bullet, 11\end{array}$ \\
\hline & & Huds./AF-29 & & & & PD & Internal & $\begin{array}{l}\text { Gastrointestinal } \\
\text { problems }\end{array}$ & $\begin{array}{l}12 \bullet, 13,14 \bullet, 15,16 \bullet \\
17,18 \bullet, 19 \bullet, 20 \bullet, 21 \bullet, \\
22 \bullet\end{array}$ \\
\hline & & & & & & TE & Internal & ${ }^{*}$ Headache & \\
\hline & & Micromeria & Chai booti & $\mathrm{H}$ & LF & JU & Internal & Digestive disorders & $1 \bullet, 2 \bullet, 3 \bullet, 4 \bullet, 5 \quad, 6 \bullet$ \\
\hline & & $\begin{array}{l}\text { biflora (Buch.- } \\
\text { Ham. ex D. }\end{array}$ & & & RT & PA & External & *Toothache & $\begin{array}{l}7 \bullet, 8 \bullet, 9 \bullet, 10 \bullet, 11 \bullet, 12 \\
13 \bullet, 14 \bullet, 15,16 \bullet, 17\end{array}$ \\
\hline & & $\begin{array}{l}\text { Don) Benth. } \\
\text { /AF-93 }\end{array}$ & & & WP & JU & Internal & *Sinus infection & $18 \bullet, 19 \bullet, 20 \bullet, 21 \bullet, 22 \bullet$ \\
\hline & & Nepeta laevigata & Jangli Bhaker & $\mathrm{H}$ & WP & PD & Internal & ${ }^{*}$ Fever, ${ }^{*}$ Headache & $1 \bullet, 2 \bullet, 3 \quad, 4 \quad, 5 \bullet, 6 \bullet$ \\
\hline & & Mazz. /AF-125 & & & SD & IN & Internal & ${ }^{*}$ Dysentery & $\begin{array}{l}13 \bullet, 14 \bullet, 15 \bullet, 16 \bullet, 17 \bullet \\
18 \bullet, 19 \bullet, 20 \bullet, 21 \bullet, 22 \bullet\end{array}$ \\
\hline & & Origanum & Ban ajwain & $\mathrm{H}$ & WP & JU & Internal & Stomachache & $1 \bullet, 2 \bullet, 3 \bullet, 4 \bullet, 5 \bullet, 6 \bullet, 7 \bullet$ \\
\hline & & vulgare L./AF-62 & & & & $\mathrm{DE}$ & External & Skin Infection & $\begin{array}{l}8 \bullet, 9 \bullet, 10 \bullet, 11 \bullet, 12, \\
13 \bullet, 14 \bullet, 15,16 \bullet, 17 \bullet,\end{array}$ \\
\hline & & & & & & EX (oil) & External & *Pain reliever & $18 \bullet, 19 \bullet, 20 \bullet, 21 \bullet, 22 \bullet$ \\
\hline & & & & & $\mathrm{SH}$ & $\mathrm{CH}$ & External & Toothache & \\
\hline & & Plectranthus & Peemar & S & LF & $\mathrm{CH}$ & External & *Toothache & $, 2 \bullet, 3 \bullet, 4 \bullet, 5 \bullet, 6 \bullet$, \\
\hline
\end{tabular}


Table 2 Medicinal uses of the reported taxa and their comparison with previous reports (Continued)

\begin{tabular}{|c|c|c|c|c|c|c|c|c|c|}
\hline \multirow{2}{*}{$\begin{array}{l}\mathrm{Sr} \\
\#\end{array}$} & \multirow[t]{2}{*}{ Family } & \multicolumn{2}{|l|}{ Nomenclature } & \multirow[t]{2}{*}{ Habit } & \multicolumn{4}{|c|}{ Medicinal uses } & \multirow[t]{2}{*}{ Previous reports } \\
\hline & & Scientific name & Local name & & $\begin{array}{l}\text { Part } \\
\text { used }\end{array}$ & Preparation & Application & Disease treated & \\
\hline & & $\begin{array}{l}\text { rugosus Wall.ex } \\
\text { Benth. /AF-34 }\end{array}$ & & & RT & $\mathrm{DE}$ & Internal & *Liver tonic & $\begin{array}{l}7 \bullet, 8,9,10 \bullet, 11 \bullet \\
12 \bullet, 13 \bullet, 14 \bullet, 15 \bullet, 16 \bullet \\
17 \bullet, 18 \bullet, 19 \bullet, 20 \bullet, 21 \bullet \\
22 \bullet\end{array}$ \\
\hline & & \multirow{4}{*}{$\begin{array}{l}\text { Prunella vulgaris } \\
\text { L./AF-72 }\end{array}$} & \multirow[t]{4}{*}{ Kathri } & \multirow[t]{4}{*}{$\mathrm{H}$} & \multirow[t]{2}{*}{ LF } & $\mathrm{DE}$ & Internal & *Sore throat & \multirow{4}{*}{$\begin{array}{l}1,2 \bullet, 3,4,5 \bullet, 6 \\
7 \bullet, 8 \bullet, 9 \bullet, 10 \bullet, 11 \bullet, 12 \bullet \\
13 \bullet, 14 \bullet, 15 \bullet, 16 \bullet, 17 \bullet \\
18 \bullet, 19 \bullet, 20,21 \bullet, 22 \bullet\end{array}$} \\
\hline & & & & & & PA & External & Skin infection & \\
\hline & & & & & \multirow[t]{2}{*}{ WP } & PD & External & *Joint pains & \\
\hline & & & & & & $\mathrm{DE}$ & Internal & Heart disease & \\
\hline & & \multirow{3}{*}{$\begin{array}{l}\text { Salvia lanata } \\
\text { Roxb./AF-126 }\end{array}$} & \multirow[t]{3}{*}{ Kathra } & \multirow[t]{3}{*}{$\mathrm{H}$} & IN & VG & Internal & ${ }^{*}$ Cough & \multirow{3}{*}{$\begin{array}{l}1 \bullet, 2 \bullet, 3,4,5 \bullet, 6 \bullet \\
7 \bullet, 8 \bullet, 9 \bullet, 10 \bullet, 11 \bullet, 12 \bullet, \\
13 \bullet, 14 \bullet, 15 \bullet, 16 \bullet, 17 \bullet, \\
18 \bullet, 19 \bullet, 20 \bullet, 21 \bullet, 22 \bullet\end{array}$} \\
\hline & & & & & $L F$ & $\mathrm{PO}$ & External & Wounds, Itching & \\
\hline & & & & & WP & EX & Internal & $\begin{array}{l}\text { *Abdominal worms, } \\
\text { *Motion }\end{array}$ & \\
\hline 26 & Lauraceae & $\begin{array}{l}\text { Machilus } \\
\text { odoratissimus } \\
\text { Nees./AF-104 }\end{array}$ & Chaan & $\mathrm{T}$ & AP & EX & Internal & $\begin{array}{l}\text { Diabetes, Epilepsy, } \\
\text { Cardiovascular diseases }\end{array}$ & $\begin{array}{l}1 \bullet, 2 \bullet, 3 \bullet, 4 \bullet, 5 \bullet, 6 \bullet, 7 \bullet, \\
8 \bullet, 9 \bullet, 10 \bullet, 11 \bullet, 12 \bullet, \\
13 \bullet, 14 \bullet, 15 \bullet, 16 \bullet, 17 \bullet, \\
18 \bullet, 19 \bullet, 20 \bullet, 21 \bullet, 22 \bullet\end{array}$ \\
\hline \multirow[t]{8}{*}{27} & \multirow[t]{8}{*}{ Lilliaceae } & \multirow{3}{*}{$\begin{array}{l}\text { Allium cepa L./ } \\
\text { AF-137 }\end{array}$} & \multirow[t]{3}{*}{ Piyaz } & \multirow[t]{3}{*}{$\mathrm{H}$} & \multirow[t]{3}{*}{$B L$} & JU & Internal & *Diarrhea & \multirow{3}{*}{$\begin{array}{l}1 \bullet, 2,3 \bullet, 4 \bullet, 5,6 \bullet, \\
7 \bullet, 8 \bullet, 9,10 \bullet, 11, \\
12 \bullet, 13,7 \bullet, 15 \bullet, 16 \\
17 \bullet, 18 \bullet, 19 \bullet, 20 \bullet, 21, \\
22 \bullet\end{array}$} \\
\hline & & & & & & $\mathrm{DE}$ & External & ${ }^{*}$ Dandruff, *Hair fall & \\
\hline & & & & & & $\mathrm{HR}$ & External & $\begin{array}{l}\text { *To remove water from } \\
\text { wounds }\end{array}$ & \\
\hline & & \multirow{5}{*}{$\begin{array}{l}\text { Allium sativum } \\
\text { L. /AF-134 }\end{array}$} & \multirow[t]{5}{*}{ Thoom } & \multirow[t]{5}{*}{$\mathrm{H}$} & $B L$ & PA & External & *Hair growth & $1 \bullet, 2,3 \bullet, 4 \bullet, 5,6 \bullet$ \\
\hline & & & & & & $\mathrm{DE}$ & Internal & ${ }^{*}$ Common cold & $\begin{array}{l}\bullet \bullet, 8 \bullet, 9,10 \bullet, 11 \bullet, 12 \bullet, \\
13 \bullet, 14,15 \bullet, 16 \bullet, 17 \bullet,\end{array}$ \\
\hline & & & & & & $\mathrm{CH}$ & External & Hypertension & $18 \bullet, 19 \bullet, 20 \bullet, 21 \bullet, 22 \bullet$ \\
\hline & & & & & & EX & External & *Joint pain & \\
\hline & & & & & LE & PD & Internal & *Stomach problems & \\
\hline 28 & Lythraceae & Punica & Darun/ Jangle & $S$ & SD & JU & Internal & *Diabetes & $1 \bullet, 2,3,4,5 \bullet, 6 \bullet$ \\
\hline & & $\begin{array}{l}\text { granatum L./AF- } \\
66\end{array}$ & annar & & LF & PA & External & Tooth pain & $\begin{array}{l}7,8,9,10,11, \\
12,13,14,15 \bullet\end{array}$ \\
\hline & & & & & FR & ET & Internal & Jaundice & $16,17 \bullet, 18 \bullet, 19 \bullet, 20 \bullet$ \\
\hline & & & & & BR & $\mathrm{DE}$ & Internal & Antithelmintic & \\
\hline 29 & Malvaceae & Malva parviflora & Sonchal & $\mathrm{H}$ & LF & VG & Internal & Constipation & $1 \bullet, 2 \bullet, 3 \bullet, 4 \bullet, 5,6 \bullet$, \\
\hline & & & & & & DE & Internal & Cough & $\begin{array}{l}7 \bullet, 8 \bullet, 9 \bullet, 10 \bullet, 11 \bullet, \\
12 \bullet 13 \bullet, 14 \bullet, 15 \bullet, 16\end{array}$ \\
\hline & & & & & WP & $\mathrm{PO}$ & External & ${ }^{*}$ To remove swelling & $17 \bullet, 18 \bullet, 19 \bullet, 20 \bullet, 21 \bullet$, \\
\hline & & & & & RT & $\mathrm{DE}$ & External & ${ }^{*}$ Dandruff & \\
\hline 30 & Meliaceae & $\begin{array}{l}\text { Melia azedarach } \\
\text { L./AF-6 }\end{array}$ & Daraik & $\mathrm{T}$ & $\mathrm{FR}$ & EX & Internal & $\begin{array}{l}\text { Diabetes, Blood } \\
\text { purification }\end{array}$ & $\begin{array}{l}1 \bullet, 2,3 \bullet, 4 \bullet, 5,6 \bullet, \\
7 \bullet, 8 \bullet, 9 \bullet, 10,11 \bullet, 12 \bullet,\end{array}$ \\
\hline & & & & & LB & EX & Internal & Blood purification & $\begin{array}{llll}13 & , 14 & , 16 \bullet \\
18 \bullet, 19 & , 20 \bullet, 21 & 17 \\
\end{array}$ \\
\hline & & & & & LF & EX & External & $\begin{array}{l}\text { *Tonic, Antiseptic, Hair } \\
\text { Fall }\end{array}$ & \\
\hline 31 & Moraceae & $\begin{array}{l}\text { Ficus carica L./ } \\
\text { AF- } 25\end{array}$ & Phagwara & S & FR & EX & Internal & $\begin{array}{l}\text { Mouth ulcers, } \\
\text { Inflammation }\end{array}$ & $\begin{array}{l}1 \bullet, 2,3,4,5,6 \bullet, \\
7 \bullet, 8 \bullet, 9 \bullet, 10 \bullet, 11,\end{array}$ \\
\hline & & & & & LF & $L X$ & External & Insect bites, Warts & $\begin{array}{ll}12 & , 13,14,15 \bullet \\
16 & , 17 \bullet, 18 \bullet, 19 \bullet, 20 \bullet\end{array}$ \\
\hline & & & & & & $\mathrm{DE}$ & Internal & Piles & $21 \bullet, 22 \bullet$ \\
\hline & & & & & FR & ET & Internal & Constipation & \\
\hline & & $\begin{array}{l}\text { Ficus palmata } \\
\text { Forssk./AF-10 }\end{array}$ & $\begin{array}{l}\text { Phagwara/ } \\
\text { Injeer }\end{array}$ & Tree & FR & ET & Internal & $\begin{array}{l}\text { *Stomach disorders, } \\
\text { Constipation }\end{array}$ & $\begin{array}{l}1 \bullet, 2,3,4,5 \bullet, 6 \bullet \\
7,8 \bullet, 9,10,11,\end{array}$ \\
\hline & & & & & LF & $L X$ & External & Skin infection, *Epilepsy & $\begin{array}{l}12 \bullet, 13 \bullet, 14 \bullet, 15 \bullet, 16 \bullet, \\
17,18 \bullet, 19 \bullet, 20 \bullet, 21 \bullet \\
22 \bullet\end{array}$ \\
\hline
\end{tabular}


Table 2 Medicinal uses of the reported taxa and their comparison with previous reports (Continued)

\begin{tabular}{|c|c|c|c|c|c|c|c|c|c|}
\hline \multirow{2}{*}{$\begin{array}{l}\text { Sr } \\
\#\end{array}$} & \multirow[t]{2}{*}{ Family } & \multicolumn{2}{|l|}{ Nomenclature } & \multirow[t]{2}{*}{ Habit } & \multicolumn{4}{|c|}{ Medicinal uses } & \multirow[t]{2}{*}{ Previous reports } \\
\hline & & Scientific name & Local name & & $\begin{array}{l}\text { Part } \\
\text { used }\end{array}$ & Preparation & Application & Disease treated & \\
\hline & & \multirow{2}{*}{$\begin{array}{l}\text { Morus alba L./ } \\
\text { AF-115 }\end{array}$} & \multirow[t]{2}{*}{ Shehoot } & \multirow[t]{2}{*}{ T } & \multirow[t]{2}{*}{$\mathrm{FR}$} & EX & Internal & *Sexual disorders & \multirow{2}{*}{$\begin{array}{l}1 \bullet, 2,3 \bullet, 4 \bullet, 5,6 \bullet, \\
7 \bullet, 8,9 \bullet, 10,11, \\
12 \bullet, 13,14 \bullet, 15 \bullet, 16, \\
17 \bullet, 18,19,20 \bullet \\
21,22 \bullet\end{array}$} \\
\hline & & & & & & JU & Internal & $\begin{array}{l}\text { *Body weakness, } \\
\text { Chest Infection }\end{array}$ & \\
\hline \multirow[t]{9}{*}{32} & \multirow[t]{9}{*}{ Oleaceae } & \multirow{6}{*}{$\begin{array}{l}\text { Jasminum } \\
\text { grandiflorum L. } \\
\text { /AF-36 }\end{array}$} & \multirow{6}{*}{$\begin{array}{l}\text { Jasmine/ } \\
\text { Chambeli }\end{array}$} & \multirow[t]{6}{*}{$\mathrm{S}$} & \multirow[t]{3}{*}{$\mathrm{FL}$} & EX & Internal & Breast cancer & \multirow{6}{*}{$\begin{array}{l}1 \bullet, 2 \bullet, 3 \bullet, 4 \bullet, 5 \bullet, 6 \bullet, 7 \bullet, \\
8 \bullet, 9 \bullet, 10 \bullet, 11 \bullet, 12 \bullet, 13 \bullet, \\
14 \bullet, 15 \bullet, 16 \bullet, 17 \bullet, 18 \bullet, \\
19 \bullet, 20 \bullet, 21 \bullet, 22 \bullet\end{array}$} \\
\hline & & & & & & $J U$ & External & Eye disorders & \\
\hline & & & & & & IN & Internal & Fever & \\
\hline & & & & & LF & $\mathrm{CH}$ & External & $\begin{array}{l}\text { Mouth ulcer, Dental } \\
\text { pain }\end{array}$ & \\
\hline & & & & & \multirow[t]{2}{*}{ RT } & EX (oil) & External & Headache & \\
\hline & & & & & & PA & External & Scabies & \\
\hline & & \multirow{3}{*}{$\begin{array}{l}\text { Olea ferruginea } \\
\text { Royle./AF-8 }\end{array}$} & \multirow[t]{3}{*}{ Kaow } & \multirow[t]{3}{*}{$\mathrm{T}$} & LF & $\mathrm{CH}$ & External & Mouth infection & \multirow{3}{*}{$\begin{array}{l}1,2,3 \bullet, 4 \bullet, 5,6 \bullet \\
7 \bullet, 8,9,10,11 \bullet, 1 \\
2 \bullet, 13,14 \bullet, 15,16 \bullet, \\
17 \bullet, 18 \bullet, 19 \bullet, 20 \bullet, 21 \bullet \\
22 \bullet\end{array}$} \\
\hline & & & & & & TE & Internal & $\begin{array}{l}\text { Digestive disorders, } \\
\text { Diabetes }\end{array}$ & \\
\hline & & & & & FR & EX & External & *Hair growth & \\
\hline \multirow[t]{2}{*}{33} & \multirow[t]{2}{*}{ Onagraceae } & \multirow{2}{*}{$\begin{array}{l}\text { Oenothera rosea } \\
\text { L'Hér.ex Aiton. } \\
\text { /AF-58 }\end{array}$} & Buti/ Seh Davi & $\mathrm{H}$ & LF & IN & Internal & Kidney disorders & $1 \bullet, 2 \bullet, 3 \bullet, 4 \bullet, 5 \bullet, 6 \bullet$ \\
\hline & & & & & RT & PD & Internal & *Body weakness & $\begin{array}{l}13 \bullet, 14 \bullet, 15 \bullet, 16 \bullet, 17 \bullet \\
18 \bullet, 19 \bullet, 20 \bullet, 21 \bullet, 22 \bullet\end{array}$ \\
\hline 34 & Oxalidaceae & Oxalis & Khati Buti & $\mathrm{H}$ & WP & ET & Internal & Jaundice & $1 \bullet, 2,3 \bullet, 4 \bullet, 5 \bullet, 6 \bullet$, \\
\hline & & $\begin{array}{l}\text { corniculata L.I } \\
\text { AF-41 }\end{array}$ & & & LF & $\mathrm{CH}$ & External & Toothache & $12,13,14 \bullet, 15,16$ \\
\hline & & & & & & $\mathrm{DE}$ & Internal & Diarrhea & $17,18 \bullet, 19,20$ \\
\hline & & & & & & ET & Internal & Blood purification & 21 \\
\hline 35 & Pinaceae & $\begin{array}{l}\text { Cedrus deodara } \\
\text { (Roxb. ex D. }\end{array}$ & Dayar & $\mathrm{T}$ & ST & EX (oil) & External & $\begin{array}{l}\text { Skin disorders } \\
\text { (eczema), *Joint pain }\end{array}$ & $\begin{array}{l}1 \bullet, 2 \bullet, 3 \bullet, 4 \bullet, 5 \bullet, 6 \\
7 \bullet, 8 \bullet, 9 \bullet, 10 \bullet, 11\end{array}$ \\
\hline & & $\begin{array}{l}\text { Don) G. Don./ } \\
\text { AF- } 61\end{array}$ & & & & & Internal & ${ }^{*}$ Digestive disorders & $\begin{array}{l}12 \bullet, 13 \bullet, 14,15 \bullet, 16 \bullet \\
17 \bullet, 18 \bullet, 19 \bullet, 20 \bullet, 21 \bullet\end{array}$ \\
\hline & & & & & ND & PA & External & $\begin{array}{l}\text { *Swelling, *To clean } \\
\text { wounds, Chest infection }\end{array}$ & $22 \bullet$ \\
\hline & & Pinus roxburghii & Chir & $\mathrm{T}$ & LF & $\mathrm{DE}$ & Internal & ${ }^{*}$ Flue & $1 \bullet, 2 \bullet, 3,4,5,6 \bullet$ \\
\hline & & & & & RS & $\mathrm{PO}$ & External & $\begin{array}{l}\text { Wound healing, } \\
{ }^{*} \text { Cracked Heels }\end{array}$ & $\begin{array}{l}12,13 \bullet, 14 \bullet, 15 \bullet, 16 \bullet, \\
17,18 \bullet, 19 \bullet, 20 \bullet, 21 \bullet,\end{array}$ \\
\hline & & & & & & & Internal & $\begin{array}{l}\text { *Joint diseases, } \\
\text { Digestive disorders, } \\
\text { *Scorpion Bite }\end{array}$ & $22 \bullet$ \\
\hline & & & & & WP & Oil & Internal & *Nose bleeding, *Flue & \\
\hline & & Pinus wallichina & Biyar & $\mathrm{T}$ & RS & $\mathrm{PO}$ & Internal & ${ }^{*}$ Cough & $1 \bullet, 2 \bullet, 3,4,5 \bullet, 6 \bullet$ \\
\hline & & & & & & & External & Wound healing & $\begin{array}{l}1 \bullet, 8 \bullet, 9 \bullet, 10 \bullet, 11 \\
13,14 \bullet, 15 \bullet, 16 \bullet, 17 \bullet,\end{array}$ \\
\hline & & & & & & IN & Internal & *Expulsion of worms & $18 \bullet, 19 \bullet, 20 \quad, 21 \bullet, 22 \bullet$ \\
\hline & & & & & & EX & Internal & $\begin{array}{l}{ }^{*} \text { Diuretic, *Kidney } \\
\text { problem }\end{array}$ & \\
\hline 36 & Plantaginaceae & Plantago & Chamchi ptra/ & $\mathrm{H}$ & $\mathrm{FL}$ & IN & Internal & Dysentery & $1 \bullet, 2,3,4,5 \bullet, 6 \bullet$ \\
\hline & & $\begin{array}{l}\text { lanceolata L. } \\
\text { AF-86 }\end{array}$ & Ispagol & & SD & PD & Internal & Diarrhea & $12 \bullet, 13,6 \bullet, 14 \bullet, 15 \bullet$ \\
\hline & & & & & LF & PA & External & Cuts, *Inflammation & $\begin{array}{l}16 \\
22 \bullet\end{array}$ \\
\hline 37 & Platanaceae & $\begin{array}{l}\text { Planatus } \\
\text { orientalis L./AF- }\end{array}$ & Chinar & $\mathrm{T}$ & BA & $J U$ & Internal & $\begin{array}{l}\text { *Snake and *Scorpion } \\
\text { bite }\end{array}$ & $\begin{array}{l}1 \bullet, 2 \bullet, 3 \bullet, 4 \bullet, 5 \bullet, 6 \bullet, 7 \bullet, \\
8 \bullet, 9 \bullet, 10 \bullet, 11 \bullet, 12 \bullet, 13 \bullet,\end{array}$ \\
\hline & & 123 & & & & $\mathrm{DE}$ & Internal & ${ }^{*}$ Dysentery & $\begin{array}{l}14,15 \bullet, 16 \bullet, 17 \bullet, 18 \bullet \\
19 \bullet, 20 \bullet 21 \bullet, 22 \bullet\end{array}$ \\
\hline & & & & & LF & PA & External & *Wound healing & \\
\hline
\end{tabular}


Table 2 Medicinal uses of the reported taxa and their comparison with previous reports (Continued)

\begin{tabular}{|c|c|c|c|c|c|c|c|c|c|}
\hline \multirow{2}{*}{$\begin{array}{l}\text { Sr } \\
\#\end{array}$} & \multirow[t]{2}{*}{ Family } & \multicolumn{2}{|l|}{ Nomenclature } & \multirow[t]{2}{*}{ Habit } & \multicolumn{4}{|c|}{ Medicinal uses } & \multirow[t]{2}{*}{ Previous reports } \\
\hline & & Scientific name & Local name & & $\begin{array}{l}\text { Part } \\
\text { used }\end{array}$ & Preparation & Application & Disease treated & \\
\hline & & & & & & DE & Internal & *Dysentery & \\
\hline & & & & & & PD & Internal & ${ }^{*}$ Teeth pain & \\
\hline \multirow[t]{26}{*}{38} & \multirow[t]{26}{*}{ Poaceae } & $\begin{array}{l}\text { Arthraxon } \\
\text { prionodes } \\
\text { (Steud.) Dandy/ } \\
\text { AF-100 }\end{array}$ & Kah & $\mathrm{H}$ & WP & DE & Internal & $\begin{array}{l}\text { Liver disease, Nervous } \\
\text { system regulator }\end{array}$ & $\begin{array}{l}1 \bullet, 2 \bullet, 3 \bullet, 4 \bullet, 5 \bullet, 6 \bullet, 7 \bullet, \\
8 \bullet, 9 \bullet, 10 \bullet, 11 \bullet, 12 \bullet, \\
13 \bullet, 14 \bullet, 15 \bullet, 16 \bullet, 17 \bullet, \\
18 \bullet, 19 \bullet, 20 \bullet, 21 \bullet, 22 \bullet\end{array}$ \\
\hline & & Aristida & Common & $\mathrm{H}$ & WP & Ash (PD) & External & Burns, Skin infection & $1 \bullet, 2 \bullet, 3 \bullet, 4 \bullet, 5 \bullet, 6 \bullet, 7 \bullet$ \\
\hline & & $\begin{array}{l}\text { cyanantha Nees } \\
\text { ex Steud./AF- } \\
122\end{array}$ & Ghass & & LE & EX & Internal & Antithelmintic & $\begin{array}{l}8 \bullet, 9 \bullet, 10 \bullet, 11 \bullet, 12 \bullet \\
13 \bullet, 14 \bullet, 15 \bullet, 16 \bullet, 17 \bullet \\
18 \bullet, 19 \bullet, 20 \bullet, 21 \bullet, 22 \bullet\end{array}$ \\
\hline & & \multirow{2}{*}{$\begin{array}{l}\text { Bromus } \\
\text { catharticus } \\
\text { Vahl./AF-68 }\end{array}$} & \multirow[t]{2}{*}{ Jarun ghass } & \multirow[t]{2}{*}{$\mathrm{H}$} & RT & EX & Internal & Purgative & \multirow{2}{*}{$\begin{array}{l}1 \bullet, 2 \bullet, 3 \bullet, 4 \bullet, 5 \bullet, 6 \bullet, 7 \bullet \\
8 \bullet, 9 \bullet, 10 \bullet, 11 \bullet, 12 \bullet, \\
13 \bullet, 14 \bullet, 15 \bullet, 16 \bullet, 17 \bullet, \\
18 \bullet, 19 \bullet, 20 \bullet, 21 \bullet, 22 \bullet\end{array}$} \\
\hline & & & & & WP & EX & External & Skin disorders & \\
\hline & & $\begin{array}{l}\text { Chrysopogon } \\
\text { gryllus (L.) Trin./ } \\
\text { AF-89 }\end{array}$ & BunchGrass & $\mathrm{H}$ & LE & DE & Internal & Fish Poisonings & $\begin{array}{l}1 \bullet, 2 \bullet, 3 \bullet, 4 \bullet, 5 \bullet, 6 \bullet, 7 \bullet \\
8 \bullet, 9 \bullet, 10 \bullet, 11 \bullet, 12 \bullet \\
13 \bullet, 14 \bullet, 15 \bullet, 16 \bullet, 17 \bullet, \\
18 \bullet, 19 \bullet, 20 \bullet, 21 \bullet, 22 \bullet\end{array}$ \\
\hline & & \multirow{4}{*}{$\begin{array}{l}\text { Cymbopogon } \\
\text { martini (Roxb.) } \\
\text { Will. Watson./ } \\
\text { AF-140 }\end{array}$} & \multirow[t]{4}{*}{$\begin{array}{l}\text { Munyara } \\
\text { Ghass }\end{array}$} & \multirow[t]{4}{*}{$\mathrm{H}$} & WP & DE & Internal & $\begin{array}{l}\text { Diarrhea, Intestinal } \\
\text { worms }\end{array}$ & \multirow{4}{*}{$\begin{array}{l}1 \bullet, 2 \bullet, 3 \bullet, 4 \bullet, 5 \bullet, 6 \bullet, 7 \bullet \\
8 \bullet, 9 \bullet, 10 \bullet, 11 \bullet, 12 \bullet, \\
13 \bullet, 14 \bullet, 15 \bullet, 16 \bullet, 17 \bullet, \\
18 \bullet, 19 \bullet, 20 \bullet, 21 \bullet, 22 \bullet\end{array}$} \\
\hline & & & & & & IN & Internal & Anorexia & \\
\hline & & & & & LE & PA & External & Skin diseases & \\
\hline & & & & & ST & PA & External & Scabies & \\
\hline & & \multirow{4}{*}{$\begin{array}{l}\text { Cynodon } \\
\text { dactylon (L.) } \\
\text { Pers./AF-18 }\end{array}$} & \multirow[t]{4}{*}{ Khabal } & \multirow[t]{4}{*}{$\mathrm{H}$} & IN & PA & External & *Skin infection & \multirow{4}{*}{$\begin{array}{l}1 \bullet, 2,3 \bullet, 4 \bullet, 5,6, \\
7,8 \bullet, 9,10,11 \bullet, \\
12 \bullet, 13 \bullet, 14 \bullet, 15 \bullet, 16, \\
17 \bullet, 18,19,20 \bullet, \\
21,22 \bullet\end{array}$} \\
\hline & & & & & WP & JU & Internal & $\begin{array}{l}{ }^{*} \text { Menstrual prolonged } \\
\text { duration, Stomach } \\
\text { acidity }\end{array}$ & \\
\hline & & & & & & & External & Eye Infection & \\
\hline & & & & & & PA & External & *Wounds healing & \\
\hline & & \multirow{3}{*}{$\begin{array}{l}\text { Dactylis } \\
\text { glomerata L./AF- } \\
107\end{array}$} & \multirow{3}{*}{ Billi Ghass } & \multirow[t]{3}{*}{ H } & LE & EX & Internal & $\begin{array}{l}\text { Kidney problem, } \\
\text { Bladder ailment }\end{array}$ & \multirow{3}{*}{$\begin{array}{l}1 \bullet, 2 \bullet, 3 \bullet, 4 \bullet, 5 \bullet, 6 \bullet, 7 \bullet \\
8 \bullet, 9 \bullet, 10 \bullet, 11 \bullet, 12 \bullet \\
13 \bullet, 14 \bullet, 15 \bullet, 16 \bullet, 17 \bullet, \\
18 \bullet, 19 \bullet, 20 \bullet, 21 \bullet, 22 \bullet\end{array}$} \\
\hline & & & & & WP & EX & Internal & Rickets & \\
\hline & & & & & $P L$ & EX & Internal & Premenstrual syndrome & \\
\hline & & \multirow{2}{*}{$\begin{array}{l}\text { Dichanthium } \\
\text { annulatum } \\
\text { (Forssk.) Stapf./ } \\
\text { AF-118 }\end{array}$} & \multirow[t]{2}{*}{$\begin{array}{l}\text { Golgen } \\
\text { beared Ghass }\end{array}$} & \multirow[t]{2}{*}{ H } & WP & EX & Internal & $\begin{array}{l}\text { *Dysentery, } \\
{ }^{*} \text { Menorrhagia }\end{array}$ & \multirow{2}{*}{$\begin{array}{l}1 \bullet, 2 \bullet, 3 \bullet, 4 \bullet, 5 \bullet, 6 \bullet, 7 \bullet, \\
8 \bullet, 9 \bullet, 10 \bullet, 11 \bullet, 12 \bullet, \\
13 \bullet, 14 \bullet, 15 \bullet, 16 \bullet, 17 \bullet, \\
18,19 \bullet, 20 \bullet, 21,22 \bullet\end{array}$} \\
\hline & & & & & RT & EX & Internal & *Blood purification & \\
\hline & & \multirow{3}{*}{$\begin{array}{l}\text { Eleusine indica } \\
\text { (L.) Gaertn./AF- } \\
131\end{array}$} & Madhani & $\mathrm{H}$ & WP & PA & External & *Stop bleeding & $1 \bullet, 2 \bullet, 3 \bullet, 4 \bullet, 5 \bullet, 6 \bullet, 7 \bullet$ \\
\hline & & & & & LF & $J U$ & Internal & *Anthelmintic & $\begin{array}{l}8 \bullet, 9 \bullet, 10 \bullet, 11 \bullet, 12 \bullet \\
13 \bullet, 14 \bullet, 15 \bullet, 16 \bullet, 17 \bullet\end{array}$ \\
\hline & & & & & RT & DE & Internal & ${ }^{*}$ Asthma & $18,19,20 \bullet, 21,22 \bullet$ \\
\hline & & $\begin{array}{l}\text { Oplismenus } \\
\text { compositus (L.) } \\
\text { P. Beauv./AF- } \\
130\end{array}$ & $\begin{array}{l}\text { Running } \\
\text { mountaingrass }\end{array}$ & $\mathrm{H}$ & $\mathrm{AP}$ & EX & External & Snake bite & $\begin{array}{l}1 \bullet, 2 \bullet, 3 \bullet, 4 \bullet, 5 \bullet, 6 \bullet, 7 \bullet, \\
8 \bullet, 9 \bullet, 10 \bullet, 11 \bullet, 12 \bullet, \\
13 \bullet, 14 \bullet, 15 \bullet, 16 \bullet, 17 \bullet, \\
18 \bullet, 19 \bullet, 20 \bullet, 21 \bullet, 22 \bullet\end{array}$ \\
\hline & & $\begin{array}{l}\text { Pennisetum } \\
\text { orientale Rich. } \\
\text { /AF-35 }\end{array}$ & $\begin{array}{l}\text { Siliak ghass/ } \\
\text { Haati Gaas }\end{array}$ & $\mathrm{H}$ & $\mathrm{AP}$ & EX & External & *Snake bite & $\begin{array}{l}1 \bullet, 2 \bullet, 3 \bullet, 4 \bullet, 5 \bullet, 6 \bullet, 7 \bullet, \\
8 \bullet, 9 \bullet, 10 \bullet, 11 \bullet, 12 \bullet, \\
13 \bullet, 14 \bullet, 15 \bullet, 16 \bullet, 17 \bullet, \\
18 \bullet, 19 \bullet, 20 \bullet, 21 \bullet, 22 \bullet\end{array}$ \\
\hline & & $\begin{array}{l}\text { Saccharum } \\
\text { spontaneum L. }\end{array}$ & Kai & $\mathrm{H}$ & WP & JU & Internal & $\begin{array}{l}\text { *Cough, }{ }^{*} \text { Abdominal } \\
\text { pain }\end{array}$ & $\begin{array}{l}1 \bullet, 2 \bullet, 3 \bullet, 4 \bullet, 5 \bullet, 6 \bullet, 7 \\
8 \bullet, 9 \bullet, 10 \bullet, 11 \bullet, 12 \bullet\end{array}$ \\
\hline & & /AF-101 & & & RT & EX & Internal & $\begin{array}{l}\text { *Piles, }{ }^{*} \text { Sexual } \\
\text { weakness, *Dyspepsia }\end{array}$ & $\begin{array}{l}13 \bullet, 14 \bullet, 15 \bullet, 16 \bullet, 17 \bullet, \\
18 \bullet, 19 \bullet, 20 \bullet, 21,22 \bullet\end{array}$ \\
\hline
\end{tabular}


Table 2 Medicinal uses of the reported taxa and their comparison with previous reports (Continued)

\begin{tabular}{|c|c|c|c|c|c|c|c|c|c|}
\hline \multirow{2}{*}{$\begin{array}{l}\text { Sr } \\
\#\end{array}$} & \multirow[t]{2}{*}{ Family } & \multicolumn{2}{|l|}{ Nomenclature } & \multirow[t]{2}{*}{ Habit } & \multicolumn{4}{|c|}{ Medicinal uses } & \multirow[t]{2}{*}{ Previous reports } \\
\hline & & Scientific name & Local name & & $\begin{array}{l}\text { Part } \\
\text { used }\end{array}$ & Preparation & Application & Disease treated & \\
\hline & & & & & & DE & Internal & *Kidney stones & \\
\hline & & $\begin{array}{l}\text { Setaria viridis (L.) } \\
\text { P. Beauv./AF- }\end{array}$ & Kera Ghass & $\mathrm{H}$ & SD & PD & Internal & $\begin{array}{l}\text { *To remove extra fats } \\
\text { from body }\end{array}$ & $\begin{array}{l}1 \bullet, 2 \bullet, 3 \bullet, 4 \bullet, 5 \bullet, 6 \bullet, \\
7 \quad, 8 \bullet, 9 \bullet, 10 \bullet, 11 \bullet, 12 \bullet,\end{array}$ \\
\hline & & & & & & DE & Internal & ${ }^{*}$ Diuretic & $\begin{array}{l}13 \bullet, 14 \bullet, 15 \bullet, 16 \bullet, 17 \bullet, \\
18 \bullet, 19 \bullet, 20 \bullet, 21 \bullet, 22 \bullet\end{array}$ \\
\hline & & & & & WP & IN & External & *Bruises & \\
\hline & & Sorghum & Barun ghass & $\mathrm{H}$ & RT & EX & Internal & Indigestion & $1 \bullet, 2 \bullet, 3 \bullet, 4 \bullet, 5 \quad, 6 \bullet$ \\
\hline & & $\begin{array}{l}\text { halepense (L.) } \\
\text { Pers./AF-102 }\end{array}$ & & & SD & PD & Internal & *Diuretic & $13 \bullet, 14 \bullet, 9 \bullet, 10 \bullet, 11 \bullet, 12 \bullet$, \\
\hline & & & & & LF & PA & External & $\begin{array}{l}\text { *Blood clotting, } \\
{ }^{*} \text { Antiseptic }\end{array}$ & $18,19 \bullet, 20 \bullet, 21 \bullet, 22 \bullet$ \\
\hline & & & & & AP & EX & Internal & *Abortion & \\
\hline \multirow[t]{14}{*}{39} & \multirow[t]{14}{*}{ Polygonaceae } & \multirow{4}{*}{$\begin{array}{l}\text { Persicaria } \\
\text { capitata (Buch.- } \\
\text { Ham. ex D. } \\
\text { Don) H. Gross./ } \\
\text { AF-125 }\end{array}$} & \multirow[t]{4}{*}{ Pink bubble } & $\mathrm{H}$ & AP & DE & Internal & Fever, Diarrhea & \multirow{4}{*}{$\begin{array}{l}1 \bullet, 2 \bullet, 3 \bullet, 4 \bullet, 5 \bullet, 6 \bullet, 7 \bullet, \\
8 \bullet, 9 \bullet, 10 \bullet, 11 \bullet, 12 \bullet, 13 \bullet, \\
14 \bullet, 15 \bullet, 16 \bullet, 17 \bullet, 18 \bullet, 19 \bullet, \\
20,21 \bullet, 22 \bullet\end{array}$} \\
\hline & & & & & & & External & *Eye diseases & \\
\hline & & & & & WP & EX & Internal & *Diuretic, ${ }^{*}$ Hypothermia & \\
\hline & & & & & RT & EX & Internal & Urinary tract infection & \\
\hline & & \multirow{5}{*}{$\begin{array}{l}\text { Polygonum } \\
\text { hydropiper L./ } \\
\text { AF-38 }\end{array}$} & \multirow{5}{*}{$\begin{array}{l}\text { Knotweed/ } \\
\text { Marsh weed }\end{array}$} & $\mathrm{H}$ & WP & DE & Internal & Menorrhagia & \multirow{5}{*}{$\begin{array}{l}1 \bullet, 2 \bullet, 3 \bullet, 4 \bullet, 5 \bullet, 6 \bullet, 7 \bullet, \\
8 \bullet, 9 \bullet, 10 \bullet, 11 \bullet, 12 \bullet, 13 \bullet, \\
14 \bullet, 15 \bullet, 16 \bullet, 17 \bullet, 18 \bullet, \\
19 \bullet, 20 \bullet, 21 \bullet, 22 \bullet\end{array}$} \\
\hline & & & & & & EX & Internal & $\begin{array}{l}\text { Joints pain, } \\
\text { Neurodegenerative } \\
\text { disorders }\end{array}$ & \\
\hline & & & & & LF & JU & Internal & Liver pain & \\
\hline & & & & & SD & PD & Internal & Laxative & \\
\hline & & & & & RT & EX & Internal & Tonic & \\
\hline & & \multirow{3}{*}{$\begin{array}{l}\text { Rumex dentatus } \\
\text { L. /AF-88 }\end{array}$} & \multirow{3}{*}{$\begin{array}{l}\text { Hullah/ Jangli } \\
\text { palak }\end{array}$} & $\mathrm{H}$ & LF & PA & External & Antiseptic & \multirow{3}{*}{$\begin{array}{l}1 \bullet, 2 \bullet, 3,4 \bullet, 5,6 \bullet, \\
7 \bullet, 8 \bullet, 9 \bullet, 10 \bullet, 11 \bullet, 12 \bullet \\
13 \bullet, 14 \bullet, 15 \bullet, 16 \bullet, 17 \\
18,19 \bullet, 20 \bullet, 21 \bullet, 22 \bullet\end{array}$} \\
\hline & & & & & & PD & External & Wound Healing & \\
\hline & & & & & & $\mathrm{RB}$ & External & $\begin{array}{l}\text { *Itching caused by } \\
\text { Utrica dioica }\end{array}$ & \\
\hline & & \multirow{2}{*}{$\begin{array}{l}\text { Rumex hastatus } \\
\text { D. Don./AF-63 }\end{array}$} & \multirow[t]{2}{*}{ Chukri/Harfali } & S & AP & RB & External & Scabies & \multirow{2}{*}{$\begin{array}{l}1 \bullet, 2 \bullet, 3,4,5 \bullet, 6 \bullet \\
7,8 \bullet, 9,10 \bullet, 11 \bullet \\
12,13,14,15, \\
16 \bullet, 17,18 \bullet, 19 \bullet, 20 \bullet \\
21,22 \bullet\end{array}$} \\
\hline & & & & & LF & EX & Internal & *Jaundice & \\
\hline \multirow[t]{6}{*}{40} & \multirow[t]{6}{*}{ Primulaceae } & \multirow{2}{*}{$\begin{array}{l}\text { Androsace } \\
\text { rotundifolia } \\
\text { Hardw./AF-14 }\end{array}$} & \multirow[t]{2}{*}{ Thandi jari } & $\mathrm{H}$ & LE & EX & Internal & $\begin{array}{l}\text { Stomach diseases, } \\
\text { Menstrual problem }\end{array}$ & \multirow{2}{*}{$\begin{array}{l}1 \bullet, 2 \bullet, 3 \bullet, 4 \bullet, 5 \bullet, 6 \bullet, 7 \bullet, \\
8 \bullet, 9 \bullet, 10 \bullet, 11 \bullet, 12 \bullet, \\
13 \bullet, 14 \bullet, 15 \bullet, 16 \bullet, 17 \bullet, \\
18 \bullet, 19 \bullet, 20 \bullet, 21 \bullet, 22 \bullet\end{array}$} \\
\hline & & & & & $\mathrm{RH}$ & EX & External & Eye disease & \\
\hline & & \multirow{4}{*}{$\begin{array}{l}\text { Myrsine africana } \\
\text { L. /AF-22 }\end{array}$} & \multirow[t]{4}{*}{ Gogel } & S & LF & IN & Internal & *Stomachache & $1 \bullet, 2 \bullet, 3 \quad, 4,5 \bullet, 6 \bullet$ \\
\hline & & & & & & DE & Internal & Blood Purifier & $\begin{array}{l}1 \bullet, 8 \bullet, 9 \bullet, 10 \bullet, 11 \bullet, 12 \bullet, \\
13 \bullet, 14 \bullet, 15 \bullet, 16 \bullet, 17 \bullet, 18 \bullet,\end{array}$ \\
\hline & & & & & FR & ET & Internal & $\begin{array}{l}\text { To remove intestinal } \\
\text { Tapeworms, *Mouth } \\
\text { Infection }\end{array}$ & $19 \bullet, 20 \bullet, 21 \bullet, 22 \bullet$ \\
\hline & & & & & & PD & Internal & *Stomachache & \\
\hline 41 & Pteridaceae & $\begin{array}{l}\text { Adiantum } \\
\text { caudatum L./AF- }\end{array}$ & Maneria & $\mathrm{H}$ & FD & EX & External & $\begin{array}{l}\text { Wound healing, Skin } \\
\text { diseases }\end{array}$ & $\begin{array}{l}1 \bullet, 2 \bullet, 3 \bullet, 4 \bullet, 5 \bullet, 6 \bullet, 7 \bullet, \\
8 \bullet, 9,10 \bullet, 11 \bullet, 12 \bullet\end{array}$ \\
\hline & & & & & & JU & Internal & $\begin{array}{l}\text { Cough, Diabetes, } \\
\text { Migraine }\end{array}$ & $\begin{array}{l}13 \bullet, 14 \bullet, 15 \bullet, 16 \bullet, 1 / \bullet, \\
18 \bullet, 19 \bullet, 20 \bullet, 21 \bullet, 22 \bullet\end{array}$ \\
\hline & & Adiantum & Hansraj & $\mathrm{H}$ & FD & $\mathrm{PO}$ & External & Snake bite & $1 \bullet, 2 \bullet, 3 \bullet, 4 \bullet, 5 \bullet, 6 \bullet, 7 \bullet$ \\
\hline & & $\begin{array}{l}\text { tenerum SW./AF- } \\
11\end{array}$ & & & & DE & Internal & $\begin{array}{l}\text { Fever, To kill intestinal } \\
\text { worms }\end{array}$ & $\begin{array}{l}8 \bullet, 9,10 \bullet, 11 \bullet, 12 \bullet, 13 \bullet, \\
14 \bullet, 15 \bullet, 16 \bullet, 17 \bullet, 18 \bullet, \\
19 \bullet, 20 \bullet, 21 \bullet, 22 \bullet\end{array}$ \\
\hline & & & & & WP & EX & Internal & $\begin{array}{l}\text { Cough, Fever, } \\
\text { Pneumonia }\end{array}$ & \\
\hline
\end{tabular}


Table 2 Medicinal uses of the reported taxa and their comparison with previous reports (Continued)

\begin{tabular}{|c|c|c|c|c|c|c|c|c|c|}
\hline \multirow{2}{*}{$\begin{array}{l}\mathrm{Sr} \\
\#\end{array}$} & \multirow[t]{2}{*}{ Family } & \multicolumn{2}{|l|}{ Nomenclature } & \multirow[t]{2}{*}{ Habit } & \multicolumn{4}{|c|}{ Medicinal uses } & \multirow[t]{2}{*}{ Previous reports } \\
\hline & & Scientific name & Local name & & $\begin{array}{l}\text { Part } \\
\text { used }\end{array}$ & Preparation & Application & Disease treated & \\
\hline & & \multirow{2}{*}{$\begin{array}{l}\text { Onychium } \\
\text { japonicum } \\
\text { (Thunb.) Kunze./ } \\
\text { AF-108 }\end{array}$} & \multirow{2}{*}{ Carrot Fern } & \multirow[t]{2}{*}{$\mathrm{H}$} & WP & EX & Internal & $\begin{array}{l}\text { Common cold, } \\
\text { Dysentery, Jaundice }\end{array}$ & \multirow{2}{*}{$\begin{array}{l}1 \bullet, 2 \bullet, 3 \bullet, 4 \bullet, 5 \bullet, 6 \bullet, 7 \bullet, \\
8 \bullet, 9 \bullet, 10 \bullet, 11 \bullet, 12 \bullet, 13 \bullet \\
14 \bullet, 15 \bullet, 16 \bullet, 17 \bullet, 18 \bullet, \\
19 \bullet, 20 \bullet, 21 \bullet, 22 \bullet\end{array}$} \\
\hline & & & & & LF & JU & External & Hair fall & \\
\hline & & \multirow{2}{*}{$\begin{array}{l}\text { Pteris cretica L. } \\
\text { /AF-60 }\end{array}$} & \multirow[t]{2}{*}{ Cretan brake } & \multirow[t]{2}{*}{$\mathrm{H}$} & $\mathrm{FD}$ & PA & External & Wound healing & \multirow{2}{*}{$\begin{array}{l}1 \bullet, 2 \bullet, 3 \bullet, 4 \bullet, 5 \bullet, 6 \bullet, 7 \bullet, \\
8 \bullet, 9 \bullet, 10 \bullet, 11 \bullet, 12 \bullet, 13 \bullet, \\
14 \bullet, 15 \bullet, 16 \bullet, 17 \bullet, 18 \bullet, \\
19 \bullet, 20 \bullet, 21 \bullet, 22 \bullet\end{array}$} \\
\hline & & & & & WP & $\mathrm{DE}$ & Internal & ${ }^{*}$ Cough & \\
\hline & & \multirow{3}{*}{$\begin{array}{l}\text { Pteris vittata L./ } \\
\text { AF-45 }\end{array}$} & \multirow[t]{3}{*}{ Nanore } & \multirow[t]{3}{*}{$\mathrm{H}$} & WP & PA & External & ${ }^{*}$ Bone Fracture & \multirow{3}{*}{$\begin{array}{l}1 \bullet, 2 \bullet, 3 \bullet, 4 \bullet, 5,6 \bullet, 7 \\
\bullet, 8 \bullet, 9 \bullet, 10 \bullet, 11 \bullet, 12 \bullet, \\
13 \bullet, 14 \bullet, 15 \bullet, 16 \bullet, 17 \bullet, 18 \bullet, \\
19 \bullet, 20 \bullet, 21 \bullet, 22 \bullet\end{array}$} \\
\hline & & & & & & EX & Internal & *Hypotonic & \\
\hline & & & & & FD & PA & External & $\begin{array}{l}\text { *Antibacterial, } \\
{ }^{*} \text { Antifungal }\end{array}$ & \\
\hline \multirow[t]{7}{*}{42} & \multirow[t]{7}{*}{ Ranunculaceae } & \multirow{2}{*}{$\begin{array}{l}\text { Clematis grata } \\
\text { Wall./AF-78 }\end{array}$} & \multirow[t]{2}{*}{ Bailari } & \multirow[t]{2}{*}{ C } & RT & EX & Internal & *Bile disorders & \multirow{2}{*}{$\begin{array}{l}1 \bullet, 2 \bullet, 3 \bullet, 4 \bullet, 5 \bullet, 6 \bullet, 7 \bullet, \\
8 \bullet, 9 \bullet, 10 \bullet, 11 \bullet, 12, \\
13 \bullet, 14 \bullet, 15 \bullet, 16 \bullet, 17 \bullet, \\
18 \bullet, 19 \bullet, 20 \bullet, 21 \bullet, 22 \bullet\end{array}$} \\
\hline & & & & & LE & TE & Internal & *Scanty lacto genesis & \\
\hline & & \multirow{3}{*}{$\begin{array}{l}\text { Ranunculus } \\
\text { arvensis L./AF- } \\
112\end{array}$} & \multirow[t]{3}{*}{ Jungli dhaniya } & \multirow[t]{3}{*}{$\mathrm{H}$} & WP & EX & Internal & $\begin{array}{l}\text { Asthma, Arthritis, Hay } \\
\text { fever }\end{array}$ & \multirow{3}{*}{$\begin{array}{l}1 \bullet, 2 \bullet, 3 \bullet, 4 \bullet, 5 \bullet, 6 \bullet, 7 \bullet, \\
8 \bullet, 9,10 \bullet, 11 \bullet, 12 \bullet, 13 \bullet, \\
14 \bullet, 15 \bullet, 16 \bullet, 17 \bullet, 18 \bullet, \\
19 \bullet, 20 \bullet, 21 \bullet, 22 \bullet\end{array}$} \\
\hline & & & & & & $\mathrm{DE}$ & Internal & To Kill Intestinal Worms & \\
\hline & & & & & & LF & EX & External & \\
\hline & & \multirow{2}{*}{$\begin{array}{l}\text { Ranunculus } \\
\text { muricatus L./AF- } \\
120\end{array}$} & Kor kandoli & $\mathrm{H}$ & $\mathrm{AP}$ & CK & Internal & Asthma & $1 \bullet, 2 \bullet, 3 \quad, 4 \quad, 5 \bullet, 6 \bullet$ \\
\hline & & & & & WP & EX & Internal & ${ }^{*}$ Gout, Fever & $\begin{array}{l}1 \bullet, 8 \bullet, 9 \bullet, 10 \bullet, 11 \bullet, 12 \bullet \\
13 \bullet, 14 \bullet, 15 \bullet, 16,17 \bullet \\
18 \bullet, 19,20 \bullet, 21 \bullet, 22 \bullet\end{array}$ \\
\hline 43 & Rosaceae & Duchesnea & Budimeva/ & $\mathrm{H}$ & FR & ET & Internal & ${ }^{*}$ Kidney stone & $1 \bullet, 2 \bullet, 3 \bullet, 4 \quad, 5 \bullet, 6 \bullet$ \\
\hline & & $\begin{array}{l}\text { indica } \\
\text { (Andrews) } \\
\text { Teschem./AF-39 }\end{array}$ & Surkh Akhra & & LF & $\mathrm{DE}$ & Internal & $\begin{array}{l}\text { Sexual weakness, } \\
\text { Mental disorders }\end{array}$ & $\begin{array}{l}7 \bullet, 8 \bullet, 9 \bullet, 10 \bullet, 11 \bullet, 12 \\
13 \bullet, 14,15 \bullet, 16 \quad, 17 \bullet \\
18 \bullet, 19 \bullet, 20 \bullet, 21 \bullet, 22 \bullet\end{array}$ \\
\hline & & Fragaria & Budi meva & $\mathrm{H}$ & RT & PD & Internal & Urinary disorder & $1 \bullet, 2 \bullet, 3 \quad, 4 \quad, 5 \bullet, 6$ \\
\hline & & $\begin{array}{l}\text { nubicola (Hook. } \\
\text { f.) Lindl.ex } \\
\text { Lacaita./AF-136 }\end{array}$ & & & FR & JU & Internal & $\begin{array}{l}\text { *Diabetes, *Sex } \\
\text { Diseases }\end{array}$ & $\begin{array}{l}7 \bullet, 8 \bullet, 9 \bullet, 10 \bullet, 11,12 \bullet \\
13,14 \bullet, 15,16 \bullet, 17 \bullet \\
18 \bullet, 19 \bullet, 20,21 \bullet, 22 \bullet\end{array}$ \\
\hline & & & & & & $\mathrm{RB}$ & External & Sunburn & \\
\hline & & $\begin{array}{l}\text { Fragaria vesca } \\
\text { L./AF-91 }\end{array}$ & Budi meva & $\mathrm{H}$ & LF & $\mathrm{DE}$ & Internal & $\begin{array}{l}\text { *Mouth ulcer, }{ }^{*} \text { Gum } \\
\text { inflammation }\end{array}$ & $\begin{array}{ll}1 & , 2 \bullet, 3 \bullet, 4 \bullet, 5 \bullet, 6 \bullet, \\
7 & , 8 \bullet, 9 \bullet, 10 \bullet, 11 \bullet\end{array}$ \\
\hline & & & & & FR & JU & Internal & $\begin{array}{l}\text { Anemia, Kidney } \\
\text { diseases }\end{array}$ & $\begin{array}{l}12 \bullet, 13 \bullet, 14 \bullet, 15 \bullet, 16 \bullet, \\
17 \bullet, 18 \bullet, 19 \bullet, 20 \bullet, 21 \bullet, \\
22 \bullet\end{array}$ \\
\hline & & $\begin{array}{l}\text { Prunus persica } \\
\text { (L.) Batsch./AF- } \\
75\end{array}$ & Aru & $\mathrm{T}$ & LF & JU & Internal & $\begin{array}{l}\text { To kill intestinal } \\
\text { worms, Whooping } \\
\text { cough }\end{array}$ & $\begin{array}{l}1 \bullet, 2 \bullet, 3,4 \bullet, 5 \bullet, 6 \bullet \\
7 \quad, 8,9,10 \bullet, 11 \\
12 \bullet, 13 \bullet, 14 \bullet, 15 \bullet, 16 \bullet\end{array}$ \\
\hline & & & & & & PD & External & Wounds & $22 \bullet$ \\
\hline & & & & & BR & $\mathrm{CH}$ & External & *Toothache & \\
\hline & & & & & $\mathrm{FL}$ & EX & Internal & $\begin{array}{l}\text { Gastrointestinal } \\
\text { problems }\end{array}$ & \\
\hline & & $\begin{array}{l}\text { Pyrus malus L./ } \\
\text { AF-98 }\end{array}$ & Saib & $\mathrm{T}$ & $F R$ & JU & Internal & $\begin{array}{l}\text { Body weakness, Joint } \\
\text { problems, *Heart } \\
\text { disease Hypertension, }\end{array}$ & $\begin{array}{l}1 \bullet, 2 \bullet, 3,4,5 \bullet, 6 \bullet, \\
7 \bullet, 8 \bullet, 9 \bullet, 10 \bullet, 11 \bullet, 12 \bullet, \\
13 \bullet, 14 \bullet, 15 \bullet, 16 \bullet, 17 \bullet,\end{array}$ \\
\hline & & & & & & PA & External & Face spots & $18 \bullet, 19 \bullet, 20 \bullet, 21 \bullet$ \\
\hline & & & & & $\mathrm{FL}$ & TE & Internal & $\begin{array}{l}\text { *Respiratory and } \\
\text { *Nerves disorders }\end{array}$ & \\
\hline & & $\begin{array}{l}\text { Pyrus pashia } \\
\text { Buch.-Ham.ex D. } \\
\text { Don. /AF-85 }\end{array}$ & Tangi & $\mathrm{T}$ & FR & ET & Internal & $\begin{array}{l}\text { Dark circles around } \\
\text { eyes }\end{array}$ & $\begin{array}{l}1 \bullet, 2 \bullet, 3 \bullet, 4 \bullet, 5 \bullet, 6 \bullet, \\
7,8 \bullet, 9 \bullet, 10 \bullet, 11 \bullet, \\
12,13 \bullet, 14 \bullet, 15 \bullet, 16 \bullet, \\
17 \bullet, 18 \bullet, 19 \bullet, 20 \bullet, 21 \bullet,\end{array}$ \\
\hline
\end{tabular}


Table 2 Medicinal uses of the reported taxa and their comparison with previous reports (Continued)

\begin{tabular}{|c|c|c|c|c|c|c|c|c|c|}
\hline \multirow{2}{*}{$\begin{array}{l}\mathrm{Sr} \\
\#\end{array}$} & \multirow[t]{2}{*}{ Family } & \multicolumn{2}{|l|}{ Nomenclature } & \multirow[t]{2}{*}{ Habit } & \multicolumn{4}{|c|}{ Medicinal uses } & \multirow[t]{2}{*}{ Previous reports } \\
\hline & & Scientific name & Local name & & $\begin{array}{l}\text { Part } \\
\text { used }\end{array}$ & Preparation & Application & Disease treated & \\
\hline & & & & & & & & & $22 \bullet$ \\
\hline & & \multirow{3}{*}{$\begin{array}{l}\text { Rosa brunonii } \\
\text { Lindl./AF-103 }\end{array}$} & \multirow{3}{*}{$\begin{array}{l}\text { Jangli Gulab/ } \\
\text { Chal }\end{array}$} & \multirow[t]{3}{*}{$S$} & BA & IN & Internal & *Blood purification & \multirow{3}{*}{$\begin{array}{l}1 \bullet, 2 \bullet, 3 \bullet, 4 \bullet, 5 \bullet, 6 \\
7 \bullet, 8 \bullet, 9 \bullet, 10 \bullet, 11 \bullet, 12 \bullet \\
13 \bullet, 14 \bullet, 15 \bullet, 16 \bullet, 17 \bullet \\
18 \bullet, 19 \bullet, 20,21 \bullet, 22 \bullet\end{array}$} \\
\hline & & & & & \multirow[t]{2}{*}{$\mathrm{FL}$} & DE & Internal & Constipation & \\
\hline & & & & & & PD & External & Skin infection & \\
\hline & & \multirow{3}{*}{$\begin{array}{l}\text { Rubus fruticosus } \\
\text { L./AF- } 54\end{array}$} & \multirow[t]{3}{*}{ Kanachi } & \multirow[t]{3}{*}{ S } & FR & EX & Internal & *Tonic & \multirow{3}{*}{$\begin{array}{l}1 \bullet, 2,3,4,5,6 \bullet, \\
7,8 \bullet, 9 \bullet, 10,11 \bullet, \\
12 \bullet, 13,14,15 \bullet, 16 \bullet, \\
17,18 \bullet, 19 \bullet, 20 \bullet, 21 \bullet, \\
22 \bullet\end{array}$} \\
\hline & & & & & & ET & Internal & *Sore throat & \\
\hline & & & & & $L F$ & IN & Internal & Diarrhea, *Bleeding & \\
\hline & & \multirow[t]{3}{*}{$\begin{array}{l}\text { Rubus ellipticus } \\
\text { Sm./AF-52 }\end{array}$} & \multirow[t]{3}{*}{ Akhrayar } & \multirow[t]{3}{*}{$S$} & FR & JU & Internal & $\begin{array}{l}\text { Fever, Cough, Sore } \\
\text { throat }\end{array}$ & \multirow{3}{*}{$\begin{array}{l}1,2 \bullet, 3 \bullet, 4 \bullet, 5,6 \bullet, \\
7 \bullet, 8 \bullet, 9 \bullet, 10 \bullet, 11 \bullet, 12 \bullet, \\
13 \bullet, 14 \bullet, 15 \bullet, 16 \bullet, 17 \bullet, \\
18 \bullet, 19 \bullet, 20 \bullet, 21 \bullet, 22 \bullet\end{array}$} \\
\hline & & & & & RT & $\mathrm{DE}$ & Internal & Fever & \\
\hline & & & & & LB & JU & Internal & *Peptic ulcer & \\
\hline & & \multirow[t]{3}{*}{$\begin{array}{l}\text { Rubus niveus } \\
\text { Thunb./AF-67 }\end{array}$} & \multirow[t]{3}{*}{ Pahvonny } & \multirow[t]{3}{*}{$\mathrm{S}$} & RT & $\mathrm{DE}$ & Internal & $\begin{array}{l}\text { Whooping cough, } \\
\text { Dysentery }\end{array}$ & \multirow{3}{*}{$\begin{array}{l}1 \bullet, 2 \bullet, 3,4,5 \bullet, 6 \bullet \\
7 \bullet, 8 \bullet, 9 \bullet, 10 \bullet, 11 \bullet, 12 \bullet \\
13 \bullet, 14 \bullet, 15 \bullet, 16 \bullet, 17 \bullet, \\
18 \bullet, 19 \bullet, 20 \bullet, 21 \bullet, 22 \bullet\end{array}$} \\
\hline & & & & & & EX & External & $\begin{array}{l}\text { Wound healing, } \\
{ }^{*} \text { Antitumor }\end{array}$ & \\
\hline & & & & & LF & IN & Internal & *Blood purifier & \\
\hline \multirow[t]{4}{*}{44} & \multirow[t]{4}{*}{ Rubiaceae } & Rubia cordifolia & Chero & C & $L F$ & PD & Internal & ${ }^{*}$ Cough & $1 \bullet, 2 \bullet, 3 \bullet, 4 \bullet, 5 \bullet, 6 \bullet, 7 \bullet$, \\
\hline & & & & & & & External & *Broken Bones & $\begin{array}{l}8 \bullet, 9,10 \bullet, 11 \bullet, 12 \bullet \\
13 \bullet, 14 \bullet, 15 \bullet, 16 \bullet, 17 \bullet\end{array}$ \\
\hline & & & & & RT & IN & Internal & $\begin{array}{l}\text { *TB, *Lung Cancer, } \\
\text { *Nervous disorders, } \\
\text { *Gout }\end{array}$ & $18 \bullet, 19 \bullet, 20 \bullet, 21 \bullet, 22 \bullet$ \\
\hline & & & & & & PA & External & Wounds & \\
\hline 45 & Rutaceae & $\begin{array}{l}\text { Zanthoxylum } \\
\text { alatum Roxb./ }\end{array}$ & Timbar & S & BA & IN & Internal & $\begin{array}{l}\text { Stomach disease, To kill } \\
\text { intestine worms, Fever }\end{array}$ & $\begin{array}{l}1 \bullet, 2 \bullet, 3 \bullet, 4 \bullet, 5 \bullet, 6 \bullet, 7 \bullet \\
8 \bullet, 9,10 \bullet, 11 \bullet, 12 \bullet\end{array}$ \\
\hline & & & & & TW & $\mathrm{RB}$ & External & Toothache & $\begin{array}{l}13 \bullet, 14 \bullet, 15 \bullet, 16 \bullet, 17 \bullet, 18 \bullet, \\
19 \bullet, 20 \bullet, 21 \bullet, 22 \bullet\end{array}$ \\
\hline & & & & & FR & JU & Internal & Indigestion, Cholera & \\
\hline & & & & & SD & EX (Oil) & External & ${ }^{*}$ Antiviral & \\
\hline & & & & & & PD & External & Toothache, Gum pain & \\
\hline 46 & Salicaceae & Salix nigra & Bees & $\mathrm{T}$ & BA & $\mathrm{PO}$ & External & To remove swelling & $1 \bullet, 2 \bullet, 3 \bullet, 4 \bullet, 5 \bullet, 6 \bullet, 7 \bullet$, \\
\hline & & & & & & PD & Internal & Dysentery, Arthritis & $\begin{array}{l}8 \bullet, 9 \bullet, 10 \bullet, 11 \bullet, 12 \bullet, \\
13 \bullet, 14 \bullet, 15 \bullet, 16 \bullet, 17 \bullet,\end{array}$ \\
\hline & & & & & LF & $\mathrm{DE}$ & Internal & To reduce pain, Fever & $18 \bullet, 19 \bullet, 20 \bullet, 21 \bullet, 22 \bullet$ \\
\hline 47 & Sapindaceae & Aesculus indica & Banakhori & $\mathrm{T}$ & BA & IN & Internal & *Fever & $1,2 \bullet, 3,4 \bullet, 5 \bullet, 6$ \\
\hline & & $\begin{array}{l}\text { (Wall. ex } \\
\text { Cambess.) }\end{array}$ & & & SD & Oil & External & ${ }^{*}$ Gout disease & $\begin{array}{l}7 \bullet, 8 \bullet, 9,10 \bullet, 11 \bullet, 12 \bullet \\
13 \bullet, 14 \bullet, 15 \bullet, 16 \bullet, 17 \bullet\end{array}$ \\
\hline & & Hook./AF-5 & & & $F R$ & PD & Internal & *Indigestion & $18 \bullet, 19 \bullet, 20 \bullet, 21 \bullet, 22 \bullet$ \\
\hline 48 & Simaroubaceae & Ailanthus & Dravia & $\mathrm{T}$ & BA & IN & Internal & Diarrhea, *'Dysentery & $1 \bullet, 2 \bullet, 3 \bullet, 4 \bullet, 5 \bullet, 6 \bullet$, \\
\hline & & $\begin{array}{l}\text { altissima (Mill.) } \\
\text { Swingle/AF-1 }\end{array}$ & & & & EX & Internal & ${ }^{*}$ Anemia & $12 \bullet, 13 \bullet, 14 \bullet, 15 \bullet, 16 \bullet$ \\
\hline & & & & & FR & JU & Internal & $\begin{array}{l}\text { *Dysentery, }{ }^{*} \text { Bloody } \\
\text { stools }\end{array}$ & $\begin{array}{l}17 \\
22 \bullet\end{array}$ \\
\hline & & & & & LE & EX/PD & Internal & *To remove Tapeworms & \\
\hline 49 & Solanaceae & Solanum nigrum & Kach Mach & $\mathrm{H}$ & FR & ET & Internal & Mouth ulcer & $1 \bullet, 2,3 \bullet, 4 \bullet, 5,6 \bullet$ \\
\hline & & & & & LF & $J U$ & Internal & ${ }^{*}$ Gout, Stomach worm & $\begin{array}{l}\bullet, 8 \bullet, 9,10,15 \bullet, 11 \bullet \\
12 \bullet, 13,14,16\end{array}$ \\
\hline & & & & & & PA & External & Skin disorders & $17,18,19 \bullet, 20 \bullet$ \\
\hline & & & & & & $\mathrm{CH}$ & External & Mouth Ulcer & \\
\hline & & & & & WP & IN & Internal & $\begin{array}{l}\text { Diuretic, Abdominal } \\
\text { disorders }\end{array}$ & \\
\hline
\end{tabular}


Table 2 Medicinal uses of the reported taxa and their comparison with previous reports (Continued)

\begin{tabular}{|c|c|c|c|c|c|c|c|c|c|}
\hline \multirow{2}{*}{$\begin{array}{l}\text { Sr } \\
\#\end{array}$} & \multirow[t]{2}{*}{ Family } & \multicolumn{2}{|l|}{ Nomenclature } & \multirow[t]{2}{*}{ Habit } & \multicolumn{4}{|c|}{ Medicinal uses } & \multirow[t]{2}{*}{ Previous reports } \\
\hline & & Scientific name & Local name & & $\begin{array}{l}\text { Part } \\
\text { used }\end{array}$ & Preparation & Application & Disease treated & \\
\hline \multirow[t]{4}{*}{50} & \multirow[t]{4}{*}{ Thymelaeaceae } & \multirow{3}{*}{$\begin{array}{l}\text { Daphne } \\
\text { papyracea } \\
\text { Wall.ex G. Don. } \\
\text { /AF-53 }\end{array}$} & \multirow[t]{3}{*}{ Lokat Patr } & \multirow[t]{3}{*}{ S } & RT & Extract & Internal & Intestinal complaints & \multirow{3}{*}{$\begin{array}{l}1 \bullet, 2 \bullet, 3 \bullet, 4 \bullet, 5 \bullet, 6 \bullet, 7 \bullet \\
8 \bullet, 9 \bullet, 10 \bullet, 11 \bullet, 12 \bullet, 13 \bullet \\
14 \bullet, 15 \bullet, 16 \bullet, 17 \bullet, 18 \bullet, 19 \\
20 \bullet, 21 \bullet, 22 \bullet\end{array}$} \\
\hline & & & & & LE & Paste & External & Swelling, Tumor & \\
\hline & & & & & ST & Paste & External & Snake bite & \\
\hline & & $\begin{array}{l}\text { Wikstroemia } \\
\text { canescens Wall. } \\
\text { ex Meisn./AF- } \\
117\end{array}$ & Chianthi & $S$ & $\mathrm{AP}$ & DE & Internal & Abortifacient & $\begin{array}{l}1 \bullet, 2 \bullet, 3 \bullet, 4 \bullet, 5 \bullet, 6 \bullet, 7 \bullet \\
8 \bullet, 9 \bullet, 10 \bullet, 11 \bullet, 12 \bullet, 13 \bullet, \\
14 \bullet, 15 \bullet, 16 \bullet, 17 \bullet, 18 \bullet, 19 \bullet \\
20 \bullet, 21 \bullet, 22 \bullet\end{array}$ \\
\hline \multirow[t]{3}{*}{51} & \multirow[t]{3}{*}{ Urticaceae } & \multirow{3}{*}{$\begin{array}{l}\text { Debregeasia } \\
\text { salicifolia (D. } \\
\text { Don) Rendle. } \\
\text { /AF-99 }\end{array}$} & \multirow[t]{3}{*}{ Sindwari } & \multirow[t]{3}{*}{ S } & $L E$ & Powder & External & Skin diseases & \multirow{3}{*}{$\begin{array}{l}1 \bullet, 2 \bullet, 3,4,5 \bullet, 6 \bullet, \\
7 \bullet, 8 \bullet, 9,10 \bullet, 11 \bullet, 12 \\
13 \bullet, 14 \bullet, 15 \bullet, 16 \bullet, 18 \bullet \\
19 \bullet, 20 \bullet, 21 \bullet, 22 \bullet\end{array}$} \\
\hline & & & & & & Infusion & Internal & *Jaundice & \\
\hline & & & & & $\mathrm{FR}$ & Juice & Internal & *Bloody diarrhea & \\
\hline 52 & Valerianacea & $\begin{array}{l}\text { Valerianella } \\
\text { muricata } \\
\text { (Steven ex } \\
\text { Roem. \& Schult.) } \\
\text { W.H. Baxter./AF- } \\
47\end{array}$ & Cornsalad & $\mathrm{H}$ & $L F$ & EX & Internal & Nerve complaints & $\begin{array}{l}1 \bullet, 2 \bullet, 3 \bullet, 4 \bullet, 5 \bullet, 6 \bullet, 7 \bullet \\
8 \bullet, 9 \bullet, 10 \bullet, 11 \bullet, 12 \bullet \\
13 \bullet, 14 \bullet, 15 \bullet, 16 \bullet, 17 \bullet, \\
18 \bullet, 19 \bullet, 20 \bullet, 22 \bullet\end{array}$ \\
\hline \multirow[t]{3}{*}{53} & \multirow[t]{3}{*}{ Verbenaceae } & \multirow{3}{*}{$\begin{array}{l}\text { Verbena } \\
\text { officinalis L./AF- } \\
138\end{array}$} & \multirow[t]{3}{*}{ Neeli Booti } & \multirow[t]{3}{*}{$\mathrm{H}$} & RT & JU & Internal & $\begin{array}{l}\text { *Stomachache, *Snake } \\
\text { bite }\end{array}$ & \multirow{3}{*}{$\begin{array}{l}1 \bullet, 2,3 \bullet, 4 \bullet, 5 \bullet, 6 \bullet, 7 \bullet \\
8 \bullet, 9 \bullet, 10 \bullet, 11 \bullet, 12 \bullet, 13 \bullet \\
14 \bullet, 15 \bullet, 16 \bullet, 17 \bullet, 18 \bullet, \\
19 \bullet, 20 \bullet, 21 \bullet, 22 \bullet\end{array}$} \\
\hline & & & & & WP & $\mathrm{DE}$ & Internal & *Dropsy & \\
\hline & & & & & $\mathrm{SH}$ & PA & External & ${ }^{*}$ Swollen gums & \\
\hline \multirow[t]{3}{*}{54} & \multirow[t]{3}{*}{ Violaceae } & \multirow[t]{3}{*}{$\begin{array}{l}\text { Viola canescens } \\
\text { Wall. /AF-81 }\end{array}$} & \multirow[t]{3}{*}{ Banafsha } & \multirow[t]{3}{*}{$\mathrm{H}$} & WP & JU & Internal & $\begin{array}{l}\text { Antipyretic, *High Blood } \\
\text { pressure, Asthma, } \\
\text { Cough, *Flue, *Eye } \\
\text { diseases, Stomachache, } \\
\text { Liver disease }\end{array}$ & \multirow[t]{3}{*}{$\begin{array}{l}1 \bullet, 2 \bullet, 3,4,5,6 \bullet, \\
7 \bullet, 8,9,10 \bullet, 11 \bullet, \\
12 \bullet, 13,14 \bullet, 15 \bullet, 16 \bullet, \\
17 \bullet, 18 \bullet, 19 \bullet, 20 \bullet, 21 \bullet, \\
22\end{array}$} \\
\hline & & & & & $\mathrm{FL}$ & $J U$ & Internal & Cough, Insomnia & \\
\hline & & & & & LF & JU & Internal & Jaundice, Cough & \\
\hline \multirow[t]{2}{*}{55} & \multirow[t]{2}{*}{ Vitaceae } & \multirow{2}{*}{$\begin{array}{l}\text { Vitis } \\
\text { jacquemontii R. } \\
\text { Parker./AF-24 }\end{array}$} & \multirow[t]{2}{*}{$\begin{array}{l}\text { Dakh/Dalore/ } \\
\text { Jungli Angoor }\end{array}$} & \multirow[t]{2}{*}{ C } & $\mathrm{FR}$ & ET & Internal & $\begin{array}{l}\text { Tonic, Constipation, } \\
\text { Laxative }\end{array}$ & $\begin{array}{l}1 \bullet, 2 \bullet, 3 \bullet, 4 \bullet, 5 \bullet, 6 \bullet, 7 \bullet \\
8 \bullet, 9 \bullet, 10 \bullet, 11 \bullet, 12 \bullet, 13 \bullet,\end{array}$ \\
\hline & & & & & ST & $J U$ & Internal & Internal fever & $19 \bullet, 20 \bullet, 21 \bullet, 22 \bullet$ \\
\hline
\end{tabular}

Habit: $H$, herbs, $S$ shrubs, $T$ trees, $C$ climber, $E$ epiphyte; 2. Part(s) used: $L E$ leaf, $F R$ fruit, $R T$ Root, $S T$ stem, $A P$ aerial Parts, ND needles, WP whole Plant, $F D$ fronds, $S D$ Seed, $F L$ flower, $B A$ bark, $B L$ bulb, $R H$ rhizome, IN inflorescence, $P L$ pollen, $T W$ twig, $S H$ shoot, $L X$ latex, $L B$ leaf bud, $G L$ galls, $B R$ branches, $F P$ floral parts, $R S$ resin; 3 . Method of preparation: $P D$ powder, $D E$ decoction, EX extract, $P A$ paste, $J U$ juice, $P O$ poultice, $I N$ infusion, $H R$ hot rubbing, $C H$ chewed, VG vegetable, $T E$ tea, $R B$ rubbing, ET eaten, $C K$ cooked, $H B$ hot beverage. $(\bullet)=$ plants with similar use(s); $(\quad)=$ plants with dissimilar use $(s) ;(\bullet)=$ plants not reported in a previous study; Condition/ailment written in bold indicate the most preferred use for a given plant; *Plant uses, which are not reported in a previous study. 1: Ahmad et al. [20]; 2: Hussain et al. [60] 3: Shaheen et al. [61]; 4: Amjad et al. [59]; 5: Ajaib et al. [62]; 6: Safeer et al. [63]; 7: Shabir et al. [64]; 8: Ahmad and Habib, [65]; 9: Qaseem et al. [66]; 10: Khan et al. [67]; 11: Wali et al. [68]; 12: ljaz et al. [69]; 13: Hussain et al. [70]; 14: Aziz et al. [71]; 15: Ahmad et al. [39]; 16: Aziz et al. [50]; 17: Gulzar et al. [45]; 18: Umair et al. [36]; 19: Zahoor et al. [72]; 20: Kayani et al. [38]; 21; Umair et al. [73]; 22: Fatima et al. [74]

species belonging to the abovementioned families contain a variety of secondary metabolites and possess significant bioactivities, pharmacological, and organoleptic properties [79]. Floristic distribution of plant species in different families was analogues to previous reports from Pakistan and around the world [20, 36, 37, 74, 80-82].

\section{Plant part(s) used}

Data presented in Fig. 5 revealed that local inhabitants of the study area use 15 different parts of plants in making recipes to treat various diseases. Among these, leaves were the most abundantly utilized plant parts with percentage contribution of $29 \%$, followed by whole plants $(21 \%)$ and root (13\%), fruit (8\%), seed (6\%), and flowers (5\%) contribution, whereas the use of aerial parts, bark, branches, stem, and latex etc. were less than 5\%. Abundant availability and easy collection or harvesting of leaves make them highly utilized plant parts [4, 61, 72, 83]. Moreover, leaves also contain a high concentration of health-beneficial secondary metabolites, phytochemicals, and essential oils, which contribute significantly to phytotherapy or treatment of various health disorders $[15,75,84]$. Likewise, roots are storage parts of plant species also rich in bioactive constituents compared to other parts $[4,85,86]$, which therefore possess more health-beneficial properties if collected in the proper time. However, previous studies revealed that majority of the researchers supported the use of leaves than roots, because eradication of roots may lead to serious 


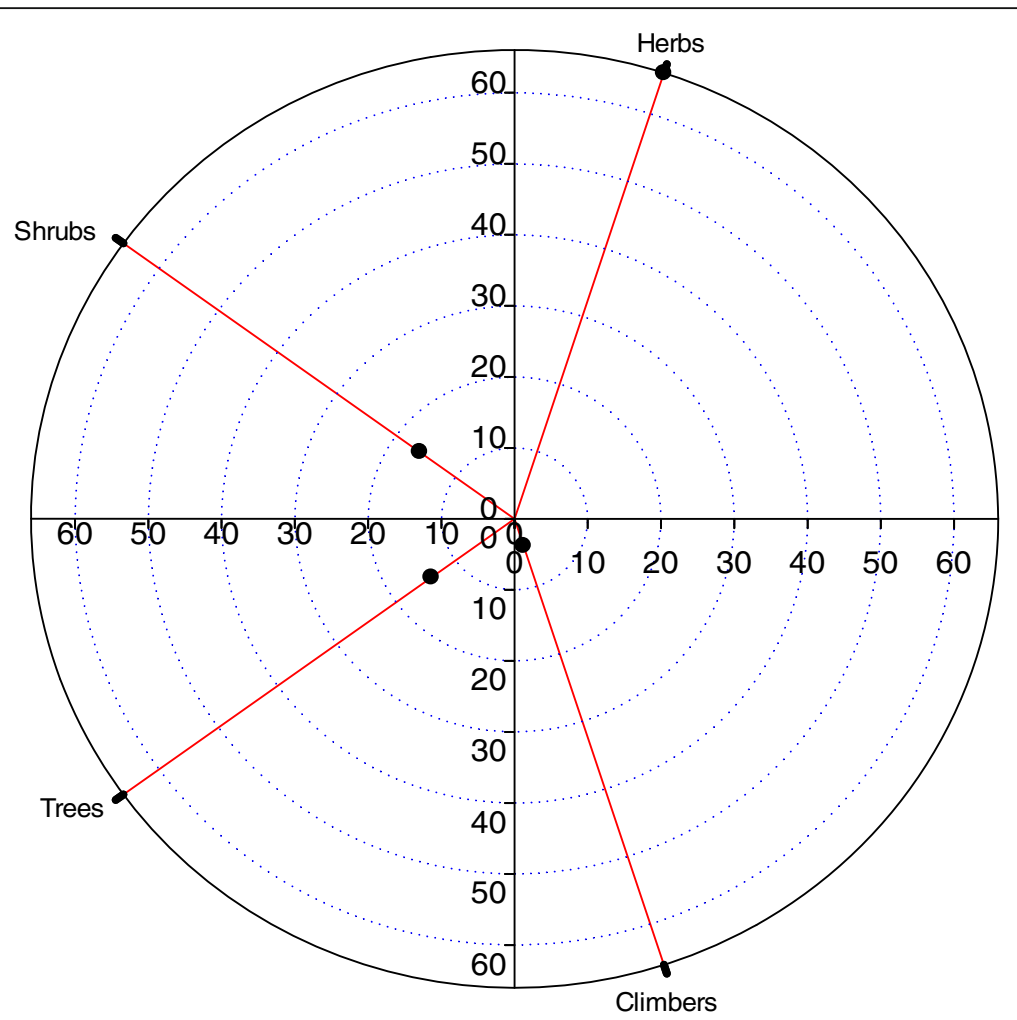

Fig. 3 Life form distribution pattern of the reported plant species in the study area

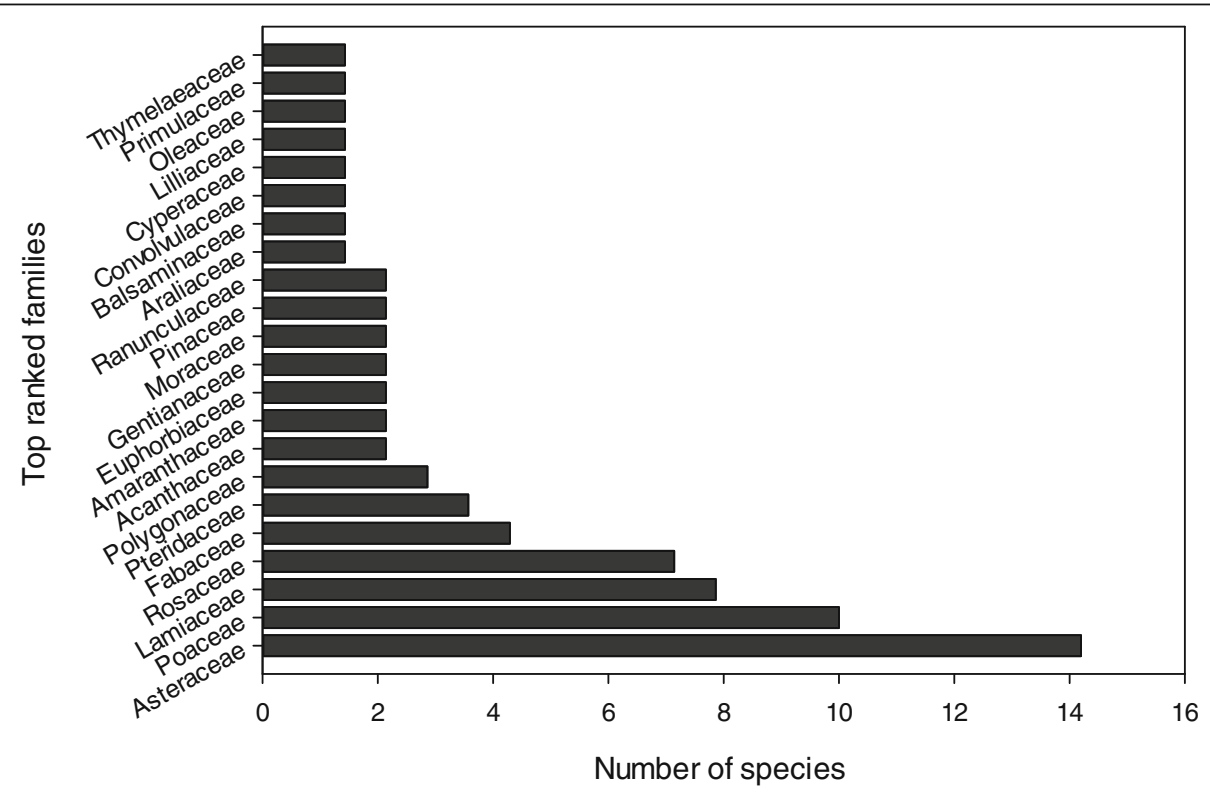

Fig. 4 Top ranked families with number of species 


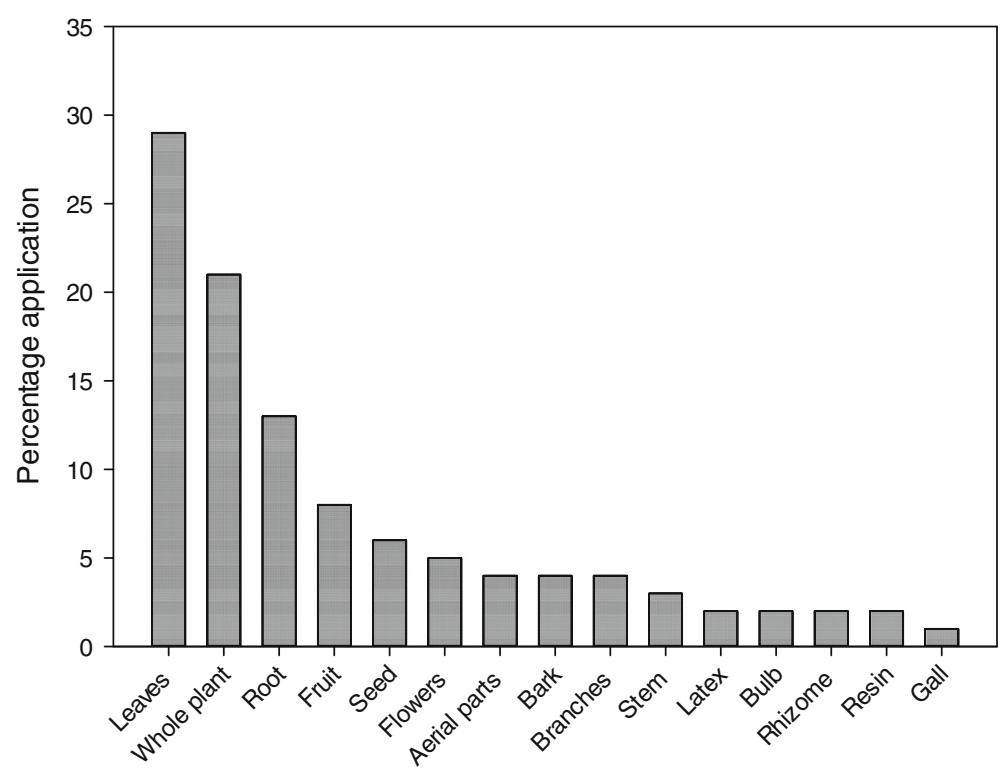

Plant parts

Fig. 5 Plant parts used in herbal recipes

conservation threats to various plant species particularly those which are highly utilized $[60,87,88]$. Moreover, it is not an easy job to collect the roots of woody and deep-rooted plants [39]. The frequent utilization of the whole plant in preparation of herbal remedies confirmed the abundant utilization of herbs in the investigated area as the whole plant can be used only in the case of herbs.

\section{Herbal preparation and administration}

Decoction was the widespread used method in the study area for herbal preparation with percentage contribution of $19 \%$, followed by extract, powder, and juice used in 18,12 , and $11 \%$ preparations of traditional recipes, respectively (Fig. 6). The frequent use of decoction had also been reported previously [36, 39, 53, 73, 81, 89, 90]. This confirms that making decoction is a very simple

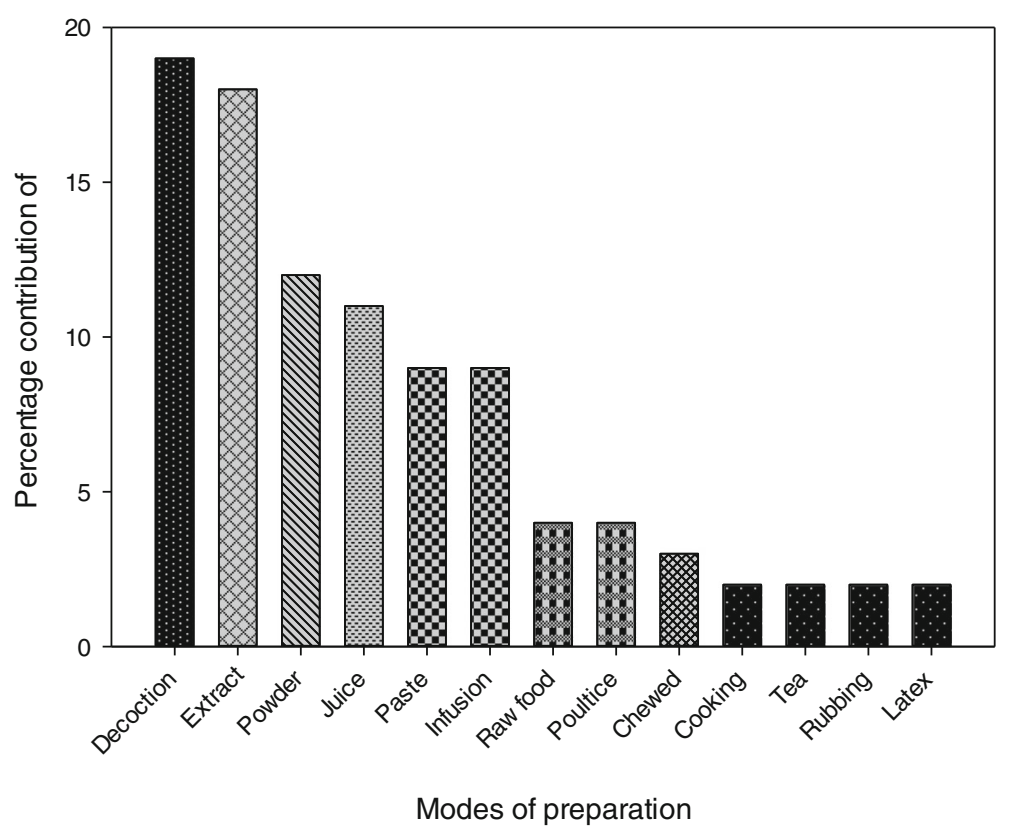

Fig. 6 Methods of preparation of herbal recipes 
and easy way used for herbal preparation with more health benefits [91]. In decoction form, the efficacy of herbal remedies increases due to the maximum extraction of health-beneficial secondary metabolites and other bioactive compounds, which is accelerated on heating [92]. Taste of medicines can be adjusted by adding honey or sugar to make it more pleasant $[39,93]$. Inhabitants of the study area use $63 \%$ of the herbal preparations as oral intake, whereas rest $37 \%$ were applied topically. These results were analogous to previous reports [36, 67, 68, 72, 94, 95]. Poultice, rubbing, and paste were common topical methods as reported in previous studies $[51,96]$. In oral mode of administration, plant materials were mainly ingested as a decoction or in powder form with water, milk, or honey. These results are analogous to the previous findings $[49,97]$. Oral intake of herbal preparation is usually effective for the treatment of internal diseases, while for external diseases, i.e., skin infections, joint pain, hemorrhoid, and stings, were treated by topical application of the drug. These observations were in agreement with previous reports [98].

\section{Informant consensus factor}

Different diseases reported from Dhirkot were classified into 16 categories to develop the consensus of informants on medicinal plants following WHO's international categorization of ailments [99]. As mentioned in Fig. 7, informant consensus factor (ICF) values ranged from 0.64 to 0.88 with the highest level of 0.88 for gastrointestinal disorders and liver diseases. Prevalence of gastrointestinal disorders is mainly attributed to poor hygiene conditions, inadequate supply of pure drinking water, and consumption of contaminated food [100, 101]. Allium cepa, Allium sativum, Mentha arvensis, Mentha longifolia, Viola canescens, Vitis jacquemontii, and Zanthoxylum alatum were among the most frequently utilized plant species to treat digestive system and liver diseases in the study area. Likewise, more consumption of a high-calorie fatty diet in the local communities and changing lifestyle could be the possible reasons of liver diseases in the study area. Our data revealed that around 90 plant species with 743 used reports were used to treat liver disorders. The plant species used to treat digestive and liver diseases have been reported as a rich source of flavonoids, toxol, vitamins, and essential oils along with other bioactive phytochemicals $[102,103]$. Additionally, inhabitants of the study area have traditional knowledge due to more interaction with these plant species, particularly used to treat digestive and liver disorders. Comparative assessment with previous studies exposed that many workers have also reported the highest ICF for digestive problems [61, 70, 71, 81, 104, 105].

The second highest ICF value viz. 0.84 was calculated for respiratory tract and throat diseases. Different factors such as sudden changes in weather, poor hygiene conditions, a high proportion of cold, moisture, germs, and spores may cause abnormalities in the respiratory track [51, 81]. Swertia cordata, Trifolium pretense, Viola

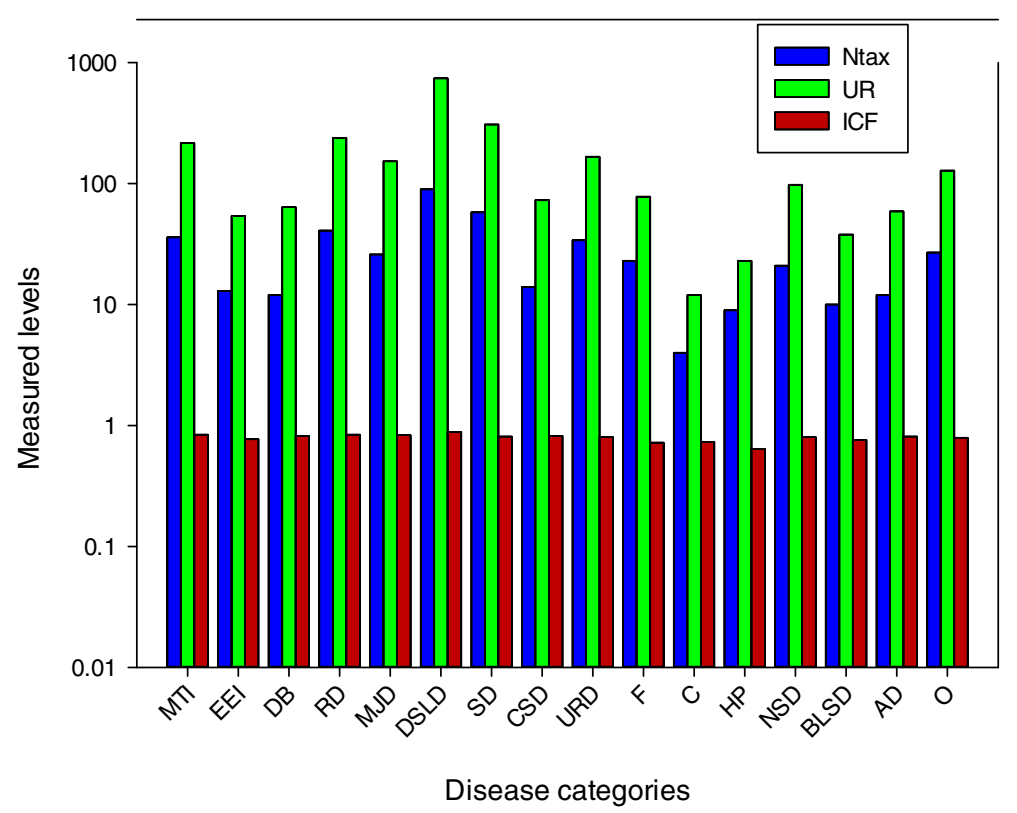

Fig. 7 Informant consensus factor of diseases with the use reports and the total number of species used. Ntax, total species used by all the informants for a group of ailment; Nur, total number of use reports in each group of disease; ICF, informant consensus factor; MTI mouth-throat infections; EEl, eye and ear infections; DB, diabetes; RD, respiratory disorders; MID, muscular and Joint disorders; DSLD, digestive system and Liver diseases; SD, skin diseases; CSD, circulatory system diseases; URD, urinary and reproductive diseases; F, fever; C, cancer; HP, hair problems; NSD, nervous system disorders; BLSD, blood and lymphatic system diseases; $A D$, antidote; $\mathrm{O}$, others 
canescens, Elaeagnus umbellate, and Achyranthes aspera were among the commonly utilized plant species for the treatment of respiratory infections. In our study, the high ICF value for skin disease might be due to the fact that local inhabitants residing in mountains at a higher altitude are more exposed to UV radiations along with other pathogenic attacks that may lead to chronic skin diseases and infections [106-108]. The most common species used to treat skin diseases were Adiantum caudatum, Ajuga bracteosa, Achillea millefolium, Berberis lycium, Cedrus deodara, Cynodon dactylon, Daphne papyracea, Debregeasia salicifolia, Ficus carica, Ficus palmate, and Gerbera gossypina.

Muscular and joint diseases are also common in the study area, which might be due to stress, minor injuries, and unhealthy food. Inhabitants of the study area use Ricinus communis, Rubia cordifolia, Salix nigra, Sarcococca saligna, and Sigesbeckia orientalis to treat joint and muscular problems. Urinary and reproductive system diseases are also common due to the unawareness and excessive use of medications. Moreover, abnormality in hormonal production, malnutrition, and environmental factor may cause reproductive disorders. The inhabitants of the study area use Saccharum spontaneum, Sarcococca saligna, Sorghum halepense, Trifolium pretense, Wikstroemia canescens, Eriophorum comosum to treat reproductive disorders. The lowest ICF value was calculated for hair problems (0.64) and 9 species including Allium cepa, Allium sativum, Melia azadarach, Olea ferruginea, and Ricinus communis were used to treat this disease with 23 use reports.

\section{Relative importance}

RI of plant species is a useful parameter to measure their adaptability. Data presented in Table 3, indicates that RI values of the reported species varied from 12.14-92.90, which were comparable with previous reports [80]. The highest RI value was calculated for Viola canescens (92.86), followed by Chenopodium ambrosioides, Pinus roxburghii, Conyza Canadensis, Jasminum grandiflorum (90.00, 82.86, 77.86, and 77.86, respectfully), whereas Pyrus malus, Galinsoga parviflora, and Hydrocotyle spp. have the same RI value (70.71 each). Plants with the highest RI indicate that they are primarily used by the inhabitants of the area and possess strong pharmacological properties [59] and their importance increases when it is used to cure more infirmities [109].

\section{Relative frequency of citation}

Relative frequency of citation (RFC) indicates the native importance of each plant species with respect to informants who reported the uses of these species [[5]. The RFC value of reported species ranged from 0.1 to 0.92 (Table 3). The highest RFC was calculated for Viola canescens (0.92) and, subsequently, Mentha arvensis (0.88), Berberis lycium (0.86), Achyranthes aspera (0.85), Taraxacum oficinale (0.85), Zanthoxylum alatum (0.82), Pinus roxburghii (0.80), Pyrus malus (0.80), Achillea millefolium (0.77), and Prunus persica (0.77). The high RFC value of these species indicates that inhabitants of the study area have a close association with these plant species and frequently use them to treat various diseases. The RFC data may contribute significantly to understand the importance of a plant species within an area, to conserve plant species having maximum RFC, and for biological, pharmacological, and phytochemical screening of such species. The high RFC of Viola canescens indicates that this species is commonly utilized by local communities to treat various health disorders. This leads to overexploitation of this species in the study area indicating a high conservation threat and may lead to extension into the future if not conserved immediately. Likewise, some plants having high RFC are rare in the study area and vice versa. For example, Rauvolfia serpentia is a rare plant in the study area but had a high FC (FC-43) value.

\section{Use value}

The use value (UV) index was used to measure the ethnomedicinal uses associated with documented medicinal plant species and is ranged from $0.11-1.7$ (Table 3). The highest UV was reported for Viola canescens (1.7), followed by Achyranthes aspera (1.3), Achillea millefolium (0.96), Mentha arvensis (0.96), Ajuga bracteosa (0.93), Pinus roxburghii (0.9), Pyrus pashia (0.90), Prunus persica (0.89), Punica granatum (0.89) Allium cepa (0.88), and Prunella vulgaris (0.88). The high usage of the reported species indicates a strong association and dependence of local communities on surrounding flora, specifically for the treatment of various diseases and as food and livelihoods [51]. Moreover, the plant species which are used excessively are assumed to be biologically more active; therefore these should be subjected to phytochemical and pharmacological screening to increase sustainable utilization and conservation of plant resources [110].

\section{Fidelity level}

FL identifies the most preferred plant species used by traditional healers to cure various diseases and shows the proportion of informants reporting the use of specific plant species. The FL level of reported species was ranged from $15.8-100 \%$. Figure 8 shows some topranked species with FL above $90 \%$. Among these, five plant species which include Berberis lyceum, Mentha arvensis, Pyrus malus, Taraxacum officinale, and Viola canescens (for wound healing, to treat gastrointestinal disorders, body weakness, diabetes, and cough, respectively) have $100 \%$ fidelity level, whereas Morus alba had 
Table 3 Quantitative analysis of ethnobotanical data

\begin{tabular}{|c|c|c|c|c|c|c|c|}
\hline Sr.\# & Scientific name & Rel. PH & Rel. BS & $\mathrm{Rl}$ & FC & RFC & UV \\
\hline 1 & Acacia nilotica & 0.50 & 0.57 & 53.57 & 31.0 & 0.42 & 0.70 \\
\hline 2 & Achillea millefolium & 0.60 & 0.57 & 58.6 & 57.0 & 0.77 & 0.96 \\
\hline 3 & Achyranthes aspera & 0.40 & 0.57 & 48.6 & 63.0 & 0.85 & 1.30 \\
\hline 4 & Adiantum caudatum & 0.50 & 0.57 & 53.6 & 29.0 & 0.40 & 0.73 \\
\hline 5 & Adiantum tenerum & 0.50 & 0.57 & 53.6 & 22.0 & 0.30 & 0.65 \\
\hline 6 & Aesculus indica & 0.30 & 0.43 & 36.4 & 19.0 & 0.26 & 0.54 \\
\hline 7 & Ailanthus altissima & 0.50 & 0.29 & 39.3 & 21.0 & 0.30 & 0.42 \\
\hline 8 & Ajuga bracteosa & 0.40 & 0.29 & 34.3 & 54.0 & 0.73 & 0.93 \\
\hline 9 & Ajuga parviflora & 0.30 & 0.43 & 36.4 & 28.0 & 0.38 & 0.55 \\
\hline 10 & Allium cepa & 0.40 & 0.43 & 41.4 & 49.0 & 0.66 & 0.88 \\
\hline 11 & Allium sativum & 0.50 & 0.71 & 60.7 & 51.0 & 0.70 & 0.82 \\
\hline 12 & Amaranthus viridis & 0.30 & 0.43 & 36.4 & 30.0 & 0.40 & 0.61 \\
\hline 13 & Androsace rotundifolia & 0.30 & 0.43 & 36.4 & 39.0 & 0.53 & 0.74 \\
\hline 14 & Arthraxon prionodes & 0.20 & 0.29 & 24.3 & 11.0 & 0.15 & 0.20 \\
\hline 15 & Aristida cyanantha & 0.30 & 0.29 & 29.3 & 20.0 & 0.30 & 0.35 \\
\hline 16 & Artemisia vulgaris & 0.20 & 0.29 & 24.3 & 53.0 & 0.72 & 0.83 \\
\hline 17 & Asplenium dalhousiae & 0.40 & 0.43 & 41.4 & 29.0 & 0.40 & 0.54 \\
\hline 18 & Berberis lycium & 0.50 & 0.71 & 60.7 & 64.0 & 0.86 & 1.30 \\
\hline 19 & Bidens biternata & 0.20 & 0.14 & 17.1 & 39.0 & 0.53 & 0.65 \\
\hline 20 & Bromus catharticus & 0.20 & 0.29 & 24.3 & 10.0 & 0.13 & 0.22 \\
\hline 21 & Campanula pallida & 0.20 & 0.14 & 17.3 & 14.0 & 0.19 & 0.26 \\
\hline 22 & Cannabis sativa & 0.20 & 0.29 & 24.3 & 24.0 & 0.32 & 0.55 \\
\hline 23 & Capsella bursa-pastoris & 0.30 & 0.43 & 36.4 & 33.0 & 0.44 & 0.62 \\
\hline 24 & Carpesium cernuum & 0.60 & 0.57 & 58.6 & 23.0 & 0.31 & 0.42 \\
\hline 25 & Cedrus deodara & 0.60 & 0.57 & 58.6 & 17.0 & 0.23 & 0.54 \\
\hline 26 & Chenopodium ambrosioides & 0.80 & 1.00 & 90.0 & 36.0 & 0.50 & 0.72 \\
\hline 27 & Chrysopogon gryllus & 0.10 & 0.14 & 12.1 & 8.0 & 0.11 & 0.11 \\
\hline 28 & Cichorium intybus & 0.70 & 0.43 & 56.4 & 39.0 & 0.53 & 0.23 \\
\hline 29 & Cirsium vulgare & 0.30 & 0.43 & 36.4 & 19.0 & 0.26 & 0.46 \\
\hline 30 & Clematis grata & 0.20 & 0.29 & 24.3 & 23.0 & 0.39 & 0.40 \\
\hline 31 & Convolvulus arvensis & 0.20 & 0.29 & 24.3 & 15.0 & 0.20 & 0.31 \\
\hline 32 & Conyza canadensis & 0.70 & 0.86 & 77.9 & 43.0 & 0.60 & 0.70 \\
\hline 33 & Cymbopogon martini & 0.50 & 0.43 & 46.4 & 13.0 & 0.20 & 0.30 \\
\hline 34 & Cynodon dactylon & 0.50 & 0.57 & 53.6 & 37.0 & 0.50 & 0.62 \\
\hline 35 & Cynoglossum lanceolatum & 0.50 & 0.29 & 39.3 & 42.0 & 0.60 & 0.76 \\
\hline 36 & Cyperus serotinus & 0.20 & 0.29 & 24.3 & 11.0 & 0.15 & 0.20 \\
\hline 37 & Dactylis glomerata & 0.50 & 0.57 & 53.6 & 23.0 & 0.31 & 0.40 \\
\hline 38 & Daphne papyracea & 0.40 & 0.57 & 48.6 & 16.0 & 0.22 & 0.32 \\
\hline 39 & Debregeasia salicifolia & 0.30 & 0.29 & 29.3 & 20.0 & 0.30 & 0.44 \\
\hline 40 & Desmodium elegans & 0.60 & 0.71 & 65.7 & 26.0 & 0.35 & 0.67 \\
\hline 41 & Dichanthium annulatum & 0.30 & 0.43 & 36.4 & 12.0 & 0.20 & 0.30 \\
\hline 42 & Dicliptera roxburghiana & 0.30 & 0.43 & 36.4 & 32.0 & 0.43 & 0.52 \\
\hline 43 & Diospyros lotus & 0.30 & 0.43 & 41.4 & 41.0 & 0.55 & 0.72 \\
\hline 44 & Dryopteris filix-mas & 0.50 & 0.43 & 46.4 & 25.0 & 0.34 & 0.46 \\
\hline
\end{tabular}


Table 3 Quantitative analysis of ethnobotanical data (Continued)

\begin{tabular}{|c|c|c|c|c|c|c|c|}
\hline Sr.\# & Scientific name & Rel. PH & Rel. BS & $\mathrm{Rl}$ & FC & RFC & UV \\
\hline 45 & Duchesnea indica & 0.30 & 0.43 & 36.4 & 29.0 & 0.40 & 0.54 \\
\hline 46 & Elaeagnus umbellata & 0.40 & 0.29 & 34.3 & 44.0 & 0.60 & 0.80 \\
\hline 47 & Eleusine indica & 0.30 & 0.43 & 36.4 & 10.0 & 0.13 & 0.20 \\
\hline 48 & Eriophorum comosum & 0.20 & 0.29 & 24.3 & 8.0 & 0.10 & 0.14 \\
\hline 49 & Euphorbia indica & 0.50 & 0.43 & 46.4 & 26.0 & 0.35 & 0.63 \\
\hline 50 & Euphorbia prostrata & 0.40 & 0.29 & 34.3 & 19.0 & 0.26 & 0.50 \\
\hline 51 & Ficus carica & 0.60 & 0.71 & 65.7 & 48.0 & 0.65 & 0.78 \\
\hline 52 & Ficus palmata & 0.50 & 0.43 & 46.4 & 53.0 & 0.72 & 0.85 \\
\hline 53 & Fragaria nubicola & 0.40 & 0.57 & 48.6 & 27.0 & 0.36 & 0.53 \\
\hline 54 & Fragaria vesca & 0.40 & 0.43 & 41.4 & 33.0 & 0.44 & 0.55 \\
\hline 55 & Galinsoga parviflora & 0.70 & 0.71 & 70.7 & 22.0 & 0.30 & 0.61 \\
\hline 56 & Gentianodes olivieri & 0.30 & 0.43 & 36.4 & 12.0 & 0.16 & 0.23 \\
\hline 57 & Gerbera gossypina & 0.30 & 0.29 & 29.3 & 29.0 & 0.40 & 0.63 \\
\hline 58 & Hedera nepalensis & 0.30 & 0.29 & 29.3 & 32.0 & 0.43 & 0.51 \\
\hline 59 & Hydrocotyle spp. & 0.70 & 0.71 & 70.7 & 26.0 & 0.35 & 0.55 \\
\hline 60 & Hypericum perforatum & 0.70 & 0.43 & 56.4 & 37.0 & 0.50 & 0.62 \\
\hline 61 & Impatiens edgeworthii & 0.30 & 0.43 & 36.4 & 11.0 & 0.15 & 0.34 \\
\hline 62 & Impatiens glandulifera & 0.30 & 0.43 & 36.4 & 19.0 & 0.26 & 0.42 \\
\hline 63 & Indigofera heterantha & 0.20 & 0.29 & 24.3 & 32.0 & 0.43 & 0.55 \\
\hline 64 & Inula spp. & 0.40 & 0.57 & 48.6 & 21.0 & 0.29 & 0.46 \\
\hline 65 & Ipomoea purpurea & 0.60 & 0.57 & 58.6 & 34.0 & 0.46 & 0.55 \\
\hline 66 & Isodon rugosus & 0.50 & 0.29 & 39.3 & 40.0 & 0.54 & 0.70 \\
\hline 67 & Jasminum grandiflorum & 0.70 & 0.86 & 77.9 & 54.0 & 0.73 & 0.82 \\
\hline 68 & Justicia vahlii & 0.10 & 0.14 & 12.1 & 9.0 & 0.12 & 0.15 \\
\hline 69 & Lespedeza juncea & 0.40 & 0.43 & 41.4 & 22.0 & 0.30 & 0.40 \\
\hline 70 & Machilus odoratissimus & 0.30 & 0.43 & 36.4 & 16.0 & 0.23 & 0.34 \\
\hline 71 & Malva parviflora & 0.40 & 0.57 & 48.6 & 44.0 & 0.60 & 0.76 \\
\hline 72 & Matricaria matricarioides & 0.50 & 0.43 & 46.4 & 23.0 & 0.31 & 0.40 \\
\hline 73 & Medicago lupulina & 0.20 & 0.29 & 24.3 & 34.0 & 0.46 & 0.54 \\
\hline 74 & Melia azedarach & 0.50 & 0.71 & 60.7 & 50.0 & 0.70 & 0.76 \\
\hline 75 & Mentha arvensis & 0.50 & 0.14 & 32.1 & 65.0 & 0.88 & 0.96 \\
\hline 76 & Mentha longifolia & 0.40 & 0.29 & 34.3 & 53.0 & 0.72 & 0.82 \\
\hline 77 & Micromeria biflora & 0.30 & 0.43 & 36.4 & 20.0 & 0.30 & 0.35 \\
\hline 78 & Morus alba & 0.30 & 0.43 & 36.4 & 38.0 & 0.51 & 0.62 \\
\hline 79 & Myriactis wallichii & 0.10 & 0.14 & 12.1 & 11.0 & 0.15 & 0.20 \\
\hline 80 & Myrsine africana & 0.40 & 0.43 & 41.4 & 53.0 & 0.72 & 0.82 \\
\hline 81 & Nepeta laevigata & 0.30 & 0.43 & 36.4 & 20.0 & 0.30 & 0.31 \\
\hline 82 & Nerium oleander & 0.30 & 0.43 & 36.4 & 43.0 & 0.60 & 0.81 \\
\hline 83 & Oenothera rosea & 0.20 & 0.29 & 24.3 & 36.0 & 0.50 & 0.60 \\
\hline 84 & Olea ferruginea & 0.40 & 0.57 & 48.6 & 52.0 & 0.76 & 0.82 \\
\hline 85 & Onychium japonicum & 0.40 & 0.43 & 41.4 & 18.0 & 0.24 & 0.42 \\
\hline 86 & Oplismenus compositus & 0.10 & 0.14 & 12.1 & 15.0 & 0.20 & 0.26 \\
\hline 87 & Origanum vulgare & 0.40 & 0.57 & 48.6 & 28.0 & 0.40 & 0.50 \\
\hline 88 & Oxalis corniculata & 0.40 & 0.43 & 41.4 & 48.0 & 0.65 & 0.74 \\
\hline
\end{tabular}


Table 3 Quantitative analysis of ethnobotanical data (Continued)

\begin{tabular}{|c|c|c|c|c|c|c|c|}
\hline Sr.\# & Scientific name & Rel. PH & Rel. BS & $\mathrm{RI}$ & FC & RFC & UV \\
\hline 89 & Parthenium hysterophorus & 0.60 & 0.71 & 65.7 & 37.0 & 0.50 & 0.61 \\
\hline 90 & Pennisetum orientale & 0.10 & 0.14 & 12.1 & 17.0 & 0.23 & 0.30 \\
\hline 91 & Persicaria capitata & 0.60 & 0.71 & 65.7 & 21.0 & 0.30 & 0.40 \\
\hline 92 & Phagnalon rupestre & 0.40 & 0.43 & 41.4 & 28.0 & 0.38 & 0.44 \\
\hline 93 & Pinus roxburghii & 0.80 & 0.86 & 82.9 & 57.0 & 0.80 & 0.90 \\
\hline 94 & Pinus wallichina & 0.50 & 0.57 & 53.6 & 51.0 & 0.70 & 0.82 \\
\hline 95 & Plantago lanceolata & 0.40 & 0.29 & 34.3 & 43.0 & 0.60 & 0.76 \\
\hline 96 & Planatus orientalis & 0.50 & 0.57 & 53.6 & 30.0 & 0.40 & 0.55 \\
\hline 97 & Plectranthus rugosus & 0.20 & 0.29 & 24.3 & 37.0 & 0.50 & 0.62 \\
\hline 98 & Polygonum hydropiper & 0.60 & 0.71 & 65.7 & 29.0 & 0.40 & 0.50 \\
\hline 99 & Prenanthes brunoniana & 0.20 & 0.14 & 17.1 & 19.1 & 0.26 & 0.32 \\
\hline 100 & Prunella vulgaris & 0.40 & 0.57 & 48.6 & 48.0 & 0.65 & 0.88 \\
\hline 101 & Prunus persica & 0.50 & 0.57 & 53.6 & 57.0 & 0.77 & 0.89 \\
\hline 102 & Pteracanthus urticifolius & 0.50 & 0.71 & 60.7 & 26.0 & 0.35 & 0.45 \\
\hline 103 & Pteris cretica & 0.20 & 0.29 & 24.3 & 8.0 & 0.10 & 0.15 \\
\hline 104 & Pteris vittata & 0.40 & 0.43 & 41.4 & 13.0 & 0.17 & 0.26 \\
\hline 105 & Punica granatum & 0.40 & 0.43 & 41.4 & 55.0 & 0.74 & 0.89 \\
\hline 106 & Pyrus malus & 0.70 & 0.86 & 77.9 & 58.0 & 0.80 & 0.87 \\
\hline 107 & Pyrus pashia & 0.20 & 0.29 & 24.3 & 53.0 & 0.72 & 0.90 \\
\hline 108 & Quercus incana & 0.50 & 0.71 & 60.7 & 55.0 & 0.74 & 0.86 \\
\hline 109 & Ranunculus arvensis & 0.50 & 0.71 & 60.7 & 21.0 & 0.28 & 0.34 \\
\hline 110 & Ranunculus muricatus & 0.30 & 0.43 & 36.4 & 12.0 & 0.22 & 0.18 \\
\hline 111 & Ricinus communis & 0.60 & 0.71 & 65.7 & 36.0 & 0.49 & 0.65 \\
\hline 112 & Rosa brunonii & 0.30 & 0.43 & 36.4 & 45.0 & 0.61 & 0.77 \\
\hline 113 & Rubia cordifolia & 0.60 & 0.71 & 65.7 & 39.0 & 0.53 & 0.62 \\
\hline 114 & Rubus fruticosus & 0.40 & 0.57 & 48.6 & 50.0 & 0.68 & 0.84 \\
\hline 115 & Rubus ellipticus & 0.40 & 0.57 & 48.6 & 42.0 & 0.56 & 0.62 \\
\hline 116 & Rubus niveus & 0.50 & 0.71 & 60.7 & 28.0 & 0.38 & 0.52 \\
\hline 117 & Rumex dentatus & 0.30 & 0.14 & 22.1 & 45.0 & 0.61 & 0.62 \\
\hline 118 & Rumex hastatus & 0.20 & 0.29 & 24.3 & 40.0 & 0.54 & 0.69 \\
\hline 119 & Saccharum spontaneum & 0.60 & 0.57 & 58.6 & 24.0 & 0.32 & 0.43 \\
\hline 120 & Salix nigra & 0.50 & 0.71 & 60.7 & 30.0 & 0.40 & 0.49 \\
\hline 121 & Salvia lanata & 0.50 & 0.43 & 46.4 & 21.0 & 0.30 & 0.44 \\
\hline 122 & Sarcococca saligna & 0.30 & 0.43 & 36.4 & 18.0 & 0.24 & 0.31 \\
\hline 123 & Setaria viridis & 0.30 & 0.43 & 36.4 & 15.0 & 0.20 & 0.26 \\
\hline 124 & Sigesbeckia orientalis & 0.60 & 0.57 & 58.6 & 33.0 & 0.44 & 0.54 \\
\hline 125 & Solanum nigrum & 0.60 & 0.71 & 65.7 & 54.0 & 0.73 & 0.85 \\
\hline 126 & Sonchus arvensis & 0.40 & 0.43 & 41.4 & 23.0 & 0.31 & 0.38 \\
\hline 127 & Sonchus oleracus & 0.60 & 0.43 & 51.4 & 29.0 & 0.40 & 0.44 \\
\hline 128 & Sorghum halepense & 0.50 & 0.57 & 53.6 & 12.0 & 0.16 & 0.20 \\
\hline 129 & Swertia cordata & 0.50 & 0.71 & 60.7 & 49.0 & 0.70 & 0.84 \\
\hline 130 & Swertia paniculata & 0.30 & 0.43 & 36.4 & 24.0 & 0.32 & 0.42 \\
\hline 131 & Tagetes minuta & 0.40 & 0.43 & 41.4 & 40.0 & 0.54 & 0.78 \\
\hline 132 & Taraxacum officinale & 0.50 & 0.29 & 39.3 & 63.0 & 0.85 & 0.86 \\
\hline
\end{tabular}


Table 3 Quantitative analysis of ethnobotanical data (Continued)

\begin{tabular}{lllllll}
\hline Sr.\# & Scientific name & Rel. PH & Rel. BS & Rl & FC & RFC \\
\hline 133 & Trifolium pratense & 0.50 & 0.57 & 53.6 & 36.0 & 0.49 \\
134 & Valerianella muricata & 0.10 & 0.14 & 12.1 & 11.0 & 0.15 \\
135 & Verbena officinalis & 0.40 & 0.57 & 48.6 & 27.0 & 0.36 \\
136 & Viburnum grandiflorum & 0.20 & 0.14 & 17.1 & 22.0 & 0.30 \\
137 & Viola canescens & 1.00 & 0.86 & 92.9 & 68.0 & 0.17 \\
138 & Vitis jacquemontii & 0.40 & 0.43 & 41.4 & 16.0 & 0.42 \\
139 & Wikstroemia canescens & 0.10 & 0.14 & 12.1 & 9.0 & 0.34 \\
140 & Zanthoxylum alatum & 0.80 & 0.57 & 68.6 & 61.0 & 0.12 \\
\hline
\end{tabular}

Rel. $P H$ relative number of pharmacological properties attributed to a single plant, Rel. BS relative number of body systems treated by a single species, $R I$ relative importance, $F C$ frequency of citation, RFC relative frequency of citation, UV use value

the lowest FL (15.8\%) and was used to treat body weakness. These findings elucidate the dominance of specific ailments in the area that are cured with different plant species, particularly having high FL [81]. Plant species having high FL values are extensively used in the area compared to those with less FL values and similar findings have already been reported [35]. These plants are used to cure different ailments since ancient times in combination with other plants or ingredients and could be considered as model plants for pharmacological screening [38]. Despite the fact that modern health facilities are accessible in the study area, local communities especially in the mountainous parts of this region still rely on medicinal plants and possess significant traditional knowledge on plant resource utilization.

\section{Novel uses}

The comparison of indigenous knowledge on medicinal plants is helpful to determine the difference between region arising due to ecological [111], historical [112], organolep,tic and phytochemical differences [71, 113]. The Jaccard index (JI) is a quantitative index used to compare the ethnobotanical data with previous reports, specifically from adjoining areas. In this study, the data was compared with 22 previously published articles. The similarity percentage with the allied area ranges from 2.08-14.9, whereas our findings were dissimilar up to 41.8 from previous data (Table 4). The highest JI value (48.4) was with data reported previous [64] from Devi Galli Azad Kashmir, Pakistan. This similarity was due to the fact that both areas have the same type of vegetation and geography along with a similarity in culture and

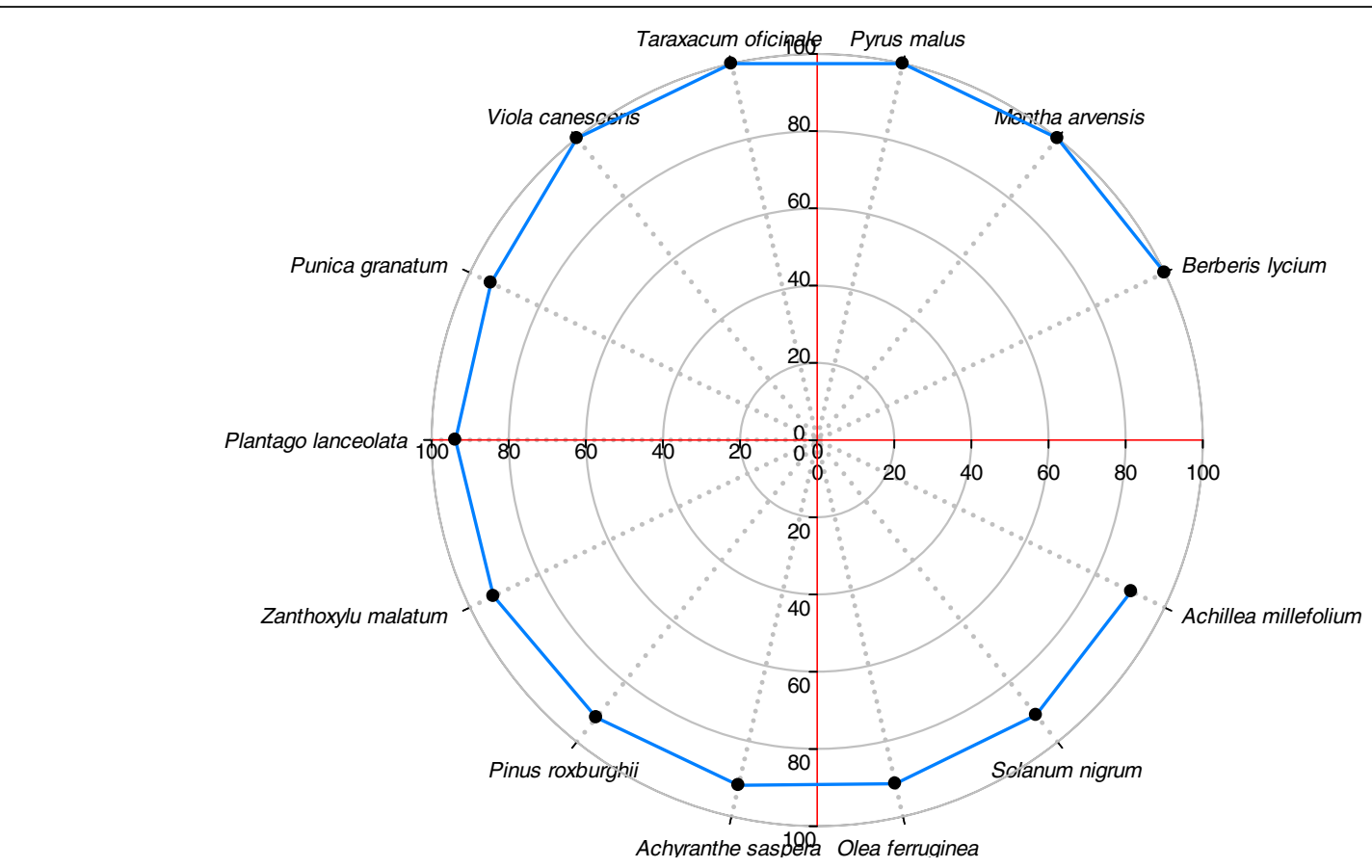

Fig. 8 Top-ranked plant species with above 90\% fidelity level 
Table 4 Jaccard index comparing the present study with previous articles

\begin{tabular}{|c|c|c|c|c|c|c|c|c|c|c|c|c|c|}
\hline $\begin{array}{l}\text { Sr. } \\
\text { no. }\end{array}$ & Study area & SY & $\mathrm{Np}$ & NRPS & NPSU & NPDU & TSCBA. & $\begin{array}{l}\text { SEAA50- } \\
18\end{array}$ & $\begin{array}{l}\text { SESA140- } \\
18\end{array}$ & $\begin{array}{l}\text { PPSU } 3 / 50 \times \\
100\end{array}$ & $\begin{array}{l}\text { PPDU } 15 / 50 \times \\
100\end{array}$ & J & C \\
\hline A & Comparison with articles from AJK & & & & & & & & & & & & \\
\hline 1 & Neelum (AJK), Pakistan & 2017 & 20 & 50 & 3 & 15 & 18 & 32 & 122 & 6.00 & 30.0 & 13.2 & [20] \\
\hline 2 & Bhimber (AJK), Pakistan & 2013 & & 97 & 5 & 20 & 25 & 72 & 115 & 5.15 & 20.62 & 15.4 & [60] \\
\hline 3 & Rawalakot, (AJK), Pakistan & 2017 & 64 & 136 & 16 & 27 & 43 & 93 & 97 & 11.8 & 19.85 & 29.3 & [61] \\
\hline 4 & $\begin{array}{l}\text { Toli Peer National Park, (AJK), } \\
\text { Pakistan }\end{array}$ & 2017 & 64 & 121 & 18 & 24 & 42 & 79 & 98 & 14.9 & 19.8 & 31.1 & [59] \\
\hline 5 & $\begin{array}{l}\text { Darguti, Tehsil khuiratta, AJK, } \\
\text { Pakistan }\end{array}$ & 2015 & & 100 & 6 & 28 & 34 & 66 & 106 & 6.00 & 28 & 24.6 & [62] \\
\hline 6 & Bagh, (AJK), Pakistan & 2017 & & 34 & 3 & 13 & 16 & 18 & 124 & 8.8 & 38.2 & 12.7 & [63] \\
\hline 7 & Devi Galli Azad Kashmir & 2017 & 135 & 98 & 6 & 41 & 47 & 51 & 93 & 6.12 & 41.8 & 48.4 & [64] \\
\hline 8 & Neelum, (AJK), Pakistan & 2014 & 100 & 59 & 2 & 19 & 21 & 38 & 119 & 3.4 & 32.2 & 15.4 & {$[65]$} \\
\hline 9 & District Kotli, (AJK),Pakistan & 2019 & 112 & 80 & 7 & 21 & 28 & 52 & 112 & 8.75 & 26.25 & 20.6 & {$[66]$} \\
\hline B & Comparison with articles from North & hern $\mathrm{P}_{\mathrm{C}}$ & akistar & & & & & & & & & & \\
\hline 10 & Dir Lower, Pakistan & 2018 & 87 & 50 & 2 & 20 & 22 & 28 & 118 & 4 & 40 & 17.7 & {$[67]$} \\
\hline 11 & Gilgit Baltistan, Pakistan & 2019 & 146 & 90 & 2 & 14 & 16 & 74 & 124 & 2.2 & 15.5 & 8.80 & [68] \\
\hline 12 & Sarban Hills, Abbottabad, Pakistan & 2016 & 134 & 74 & 4 & 17 & 21 & 53 & 119 & 5.4 & 22.9 & 13.9 & [69] \\
\hline 13 & Northern Pakistani Afghan borders & 2018 & 108 & 92 & 2 & 23 & 25 & 67 & 115 & 2.8 & 25 & 16.0 & {$[70]$} \\
\hline 14 & Bajaur Agency, Pakistan & 2017 & 108 & 79 & 5 & 18 & 23 & 55 & 116 & 6.33 & 22.8 & 15.5 & [71] \\
\hline 15 & Chail Valley, District Swat, Pakistan & 2014 & 142 & 50 & 7 & 10 & 17 & 33 & 123 & 14 & 20 & 12.2 & [39] \\
\hline 16 & South Waziristan agency, Pakistan & 2016 & 113 & 82 & 4 & 17 & 21 & 61 & 119 & 4.88 & 20.7 & 13.2 & {$[50]$} \\
\hline 17 & Malakand, KPK, Pakistan & 2019 & & 50 & 3 & 14 & 17 & 33 & 123 & 6 & 28 & 12.2 & [45] \\
\hline C & Comparison with articles from whol & le Pakis & & & & & & & & & & & \\
\hline 18 & Hafizabad district, Punjab, Pakistan & 2107 & 166 & 85 & 7 & 11 & 18 & 67 & 122 & 8.2 & 12.9 & 10.5 & [36] \\
\hline 19 & District Sheikupura, Pakistan & 2017 & 400 & 96 & 2 & 13 & 15 & 81 & 125 & 2.08 & 13.54 & 7.85 & {$[72]$} \\
\hline 20 & $\begin{array}{l}\text { Alpine and Sub-alpine regions of } \\
\text { Pakistan }\end{array}$ & 2015 & 290 & 125 & 3 & 12 & 15 & 110 & 125 & 2.4 & 9.6 & 6.80 & [38] \\
\hline 21 & $\begin{array}{l}\text { Chenab riverine, Punjab province } \\
\text { Pakistan }\end{array}$ & 2019 & 321 & 129 & 7 & 13 & 20 & 109 & 120 & 5.4 & 10.1 & 9.60 & [73] \\
\hline 22 & Central Punjab-Pakistan & 2017 & 197 & 72 & 2 & 7 & 9 & 63 & 131 & 2.8 & 9.7 & 4.90 & [74] \\
\hline
\end{tabular}

SY study year, Np number of participants, NRPs number of reported plant species, NPSU number of planst with similar uses, NPDU number of plants with different uses, TSCBA total species common in both area, SEAA species enlicted in aligned areas, SESA species enlisted only in study area, PPSU percentage of plant with similar uses, PPDU percentage of plant with different uses, Jl Jaccard index, C citation

cross-cultural exchange of traditional knowledge among communities. Conversely, our data depicted the lowest similarity $(\mathrm{JI}=2.08)$ with reported ethnomedicinal uses of plant species from Central Punjab, Pakistan [7]. These variations might be due to cultural diversity, geoclimatic conditions, habitat structure, and change on vegetation types of bath areas. More specifically, the origin and culture of local communities have a significant influence on ethno-ecological knowledge.

Comparative analysis of present findings with reported literature revealed some new uses of plant species, which have rarely been documented so far from this region, such as the stem ash of A. nilotica is used to treat eye infections. Leaves of $A$. bracteosa, A. rotundifolia, B. lyceum, $I$. rugosus, $P$. roxburghii, and $T$. officinale are used to cure stomach disorders, menstrual problems, and flu and to heal wounds in the form of different formulations (decoction, extract, paste, and powder). Likewise, inhabitants of the study area use fruits of F. nubicola, M. azedarach, M. africana, O. ferruginea, and S. nigrum for the treatment of diabetes and mouth infections, to remove intestinal worms, and for hair growth (Table 2). Consequently, documenting and comparing such information reflects the considerable intensity of knowledge among local communities, which can provide a novel source of remedial preparation [114] and indicates the high degree of ethnomedicinal novelty in the study area [20,36].

\section{Conclusions}

Due to its unique geography and diverse climatic conditions, Dhirkot and its allied areas harbor rich botanical and cultural diversity. Though inhabitants of this area 
have a strong association with surrounding flora and fauna, ethnomedicinal knowledge is at an extreme risk of extinction as it is mainly restricted to traditional healers, midwives, and older people. Consequently, there is a dire need to avoid the extinction of this ethnobotanical heritage that could be attained by the involvement of concerned authorities, conservation managers, and academia. Furthermore, high-value medicinal plant species of this area not only could contribute significantly in the livelihood of the future generations, particularly of this region, but also be a rich source of biomass supply for pharmaceutical industries.

\section{Acknowledgements}

Local inhabitants of the study area are gratefully acknowledged for sharing valuable information.

\section{Declaration}

Ethnomedicinal and cultural practices of mammals and birds in the vicinity of river Chenab, Punjab-Pakistan.

\section{Authors' contributions}

AF designed the study and conducted field survey; MSA supervised the project; KA, MA, MU helped in data analysis, interpretation, and preparation/ correction of the final draft. All the authors critically read this article and approved it as the final manuscript.

\section{Funding}

This paper is a part of a master's thesis by student Miss Asia Farooq (first author). However, no funding was provided by any source to conduct this survey.

\section{Availability of data and materials}

All data have already been included in the manuscript.

\section{Ethics approval and consent to participate}

The present study is purely based on a field survey instead of human or animal trails. Therefore, ethical approval and consent to participate is not applicable. However, formal consent was received from informants regarding data collection and publication; then, the Participatory rural appraisal (PRA) approach as mentioned in the Kyoto Protocol was applied with the consent of the informant. Ethical guidelines of the International Society of Ethnobiology (http://www.ethnobiology.net/) were strictly followed.

\section{Consent for publication}

The present paper does not contain any individual person's data; therefore, this section is not applicable to our study.

\section{Competing interests}

The authors declare that they have no competing interests.

\section{Author details}

'Department of Botany, Women University of Azad Jammu \& Kashmir, Bagh, Pakistan. ${ }^{2}$ Department of Environment Sciences, COMSATS University Islamabad, Abbottabad Campus 22060, Pakistan. ${ }^{3}$ Department of Zoology, Women University of Azad Jammu \& Kashmir, Bagh, Pakistan. ${ }^{4}$ School of Agriculture and Biology, Shanghai Jiao Tong University, Shanghai 200240, China.

Received: 7 May 2019 Accepted: 6 August 2019

Published online: 30 August 2019

\section{References}

1. Heinrich M, Ankli A, Frei B, Weimann C, Sticher O. Medicinal plants in Mexico: healers' consensus and cultural importance. Soc Sci Med. 1998;47(11):1859-71.

2. Panyaphu K, Van On T, Sirisa-Ard P, Srisa-Nga P, Chansakaow S, Nathakarnkitkul S. Medicinal plants of the mien (Yao) in northern Thailand and their potential value in the primary healthcare of postpartum women. J Ethnopharmacol. 2011;135(2):226-37.

3. Heinrich M. Ethnobotany and its role in drug development. Phytother Res. 2000;14(7):479-88.

4. Srithi K, Balslev H, Wangpakapattanawong P, Srisanga P, Trisonthi C. Medicinal plant knowledge and its erosion among the mien (Yao) in northern Thailand. J Ethnopharmacol. 2009;123(2):335-42.

5. Vitalini S, Iriti M, Puricelli C, Ciuchi D, Segale A, Fico G. Traditional knowledge on medicinal and food plants used in Val san Giacomo (Sondrio, Italy) —an alpine ethnobotanical study. J Ethnopharmacol. 2013;145(2):517-29.

6. Baydoun S, Chalak L, Dalleh H, Arnold N. Ethnopharmacological survey of medicinal plants used in traditional medicine by the communities of mount Hermon, Lebanon. J Ethnopharmacol. 2015;173:139-56.

7. Organization WH. Traditional medicine-growing needs and potential. WHO policy perspectives on medicine, no. 2. In.: WHO/EBM/2002. Geneva: WHO; 2002.

8. Haq I. Safety of medicinal plants. Pak J Med Res. 2004;43(4):203-10.

9. Shaikh BT, Hatcher J. Complementary and alternative medicine in Pakistan: prospects and limitations. Evid Based Complement Alternat Med. 2005;2(2):139-42.

10. Schippmann U, Leaman DJ, Cunningham A: Impact of cultivation and gathering of medicinal plants on biodiversity: global trends and issues. Biodiversity and the ecosystem approach in agriculture, forestry and fisheries 2002.

11. Shinwari ZK. Medicinal plants research in Pakistan. J Med Plants Res. 2010;4(3):161-76.

12. Shaikh SH, Malik F, James $H$, Abdul H. Trends in the use of complementary and alternative medicine in Pakistan: a population-based survey. J Altern Complement Med. 2009;15(5):545-50.

13. Amjad MS, Arshad M. Ethnobotanical inventory and medicinal uses of some important woody plant species of Kotli, Azad Kashmir, Pakistan. Asian Pac J Trop Biomed. 2014;4(12):952-8.

14. Quave $\mathrm{CL}$, Pieroni A. A reservoir of ethnobotanical knowledge informs resilient food security and health strategies in the Balkans. Nature Plants. 2015;1(2):14021.

15. Amjad MS, Arshad M, Qureshi R. Ethnobotanical inventory and folk uses of indigenous plants from Pir Nasoora National Park, Azad Jammu and Kashmir. Asian Pac J Trop Biomed. 2015;5(3):234-41.

16. Khan M, Khan MA, Mujtaba G, Hussain M. Ethnobotanical study about medicinal plants of Poonch valley Azad Kashmir. J animal plant Sci. 2012;22:493-500.

17. Ishtiaq CM, Khan M, Hanif W. An ethnomedicinal inventory of plants used for family planning and sex diseases treatment in Samahni valley,(AK) Pakistan. Pak J Biol Sci. 2006:9(14):2546-55.

18. Akbar K. Potential impacts of climate change on plant diversity of hilly areas of Azad Kashmir and their mitigation: a review. J Mt Area Res. 2017;2:37-44.

19. Ajaib M, Khan Z, Khan N, Wahab M. Ethnobotanical studies on useful shrubs of district Kotli, Azad Jammu \& Kashmir, Pakistan. Pak J Bot. 2010;42(3):1407-15.

20. Ahmad KS, Hamid A, Nawaz F, Hameed M, Ahmad F, Deng J, Akhtar N, Wazarat A, Mahroof S. Ethnopharmacological studies of indigenous plants in Kel village, Neelum Valley, Azad Kashmir, Pakistan. J Ethnobiol Ethnomed. 2017;13(1):68.

21. Gorsi M, Shahzad R: Medicinal uses of plants with particular reference to the people of Dhirkot. Azad Jammu and Kashmir. Asian Journal of Plant Sciences 2002.

22. Khan RN. Distribution and habitat preference of small mammals in Dhirkot, AJK. M.Sc. Thesis. University of AJK, Muzaffarabad. Muzaffarabad: Thesis University of AJK; 2002.

23. Heinrich M, Edwards S, Moerman DE, Leonti M. Ethnopharmacological field studies: a critical assessment of their conceptual basis and methods. J Ethnopharmacol. 2009;124(1):1-17.

24. Jain SK, Rao RR: A handbook of field and herbarium methods. New Delhi: today and tomorrow's Printers and Publishers xvi, 157p-llus General (KR, 197700062) 1977.

25. Nasir E, Ali S: Flora of West Pakistan Department of Botany. University of Karachi, Karachi 1971, 2007:112-115.

26. Ali S, Qaiser M. Flora of Pakistan 194-210. Karachi: Department of Botany, University of Karachi; 1993.

27. Chase MW, Christenhusz M, Fay M, Byng J, Judd WS, Soltis D, Mabberley D, Sennikov A, Soltis PS, Stevens PF. An update of the angiosperm phylogeny group classification for the orders and families of flowering plants: APG IV. Bot J Linn Soc. 2016;181(1):1-20.

28. Gardens RB, Kew MBG: The Plant List, Version 1.1. Recuperado el 2013, 2.

29. Kadam P, Bhalerao S. Sample size calculation. Int J Ayurveda Res. 2010;1 (1):55-70.

30. Edwards S, Nebel S, Heinrich M. Questionnaire surveys: methodological and epistemological problems for field-based ethnopharmacologists. J Ethnopharmacol. 2005;100(1-2):30-6.

31. Vijayakumar S, Yabesh JM, Prabhu S, Manikandan R, Muralidharan B. Quantitative ethnomedicinal study of plants used in the Nelliyampathy hills of Kerala, India. J Ethnopharmacol. 2015;161:238-54. 
32. Šavikin K, Zdunić G, Menković N, Živković J, Ćujić N, Tereščenko M, Bigović D. Ethnobotanical study on traditional use of medicinal plants in SouthWestern Serbia, Zlatibor district. J Ethnopharmacol. 2013;146(3):803-10.

33. González-Tejero M, Casares-Porcel M, Sánchez-Rojas C, Ramiro-Gutiérrez J, Molero-Mesa J, Pieroni A, Giusti M, Censorii E, De Pasquale C, Della A. Medicinal plants in the Mediterranean area: synthesis of the results of the project Rubia. J Ethnopharmacol. 2008;1 16(2):341-57.

34. Khan MPZ, Ahmad M, Zafar M, Sultana S, Ali MI, Sun H. Ethnomedicinal uses of edible wild fruits (EWFs) in Swat Valley, northern Pakistan. J Ethnopharmacol. 2015;173:191-203.

35. Friedman J, Yaniv Z, Dafni A, Palewitch D. A preliminary classification of the healing potential of medicinal plants, based on a rational analysis of an ethnopharmacological field survey among Bedouins in the Negev Desert, Israel. J Ethnopharmacol. 1986;16(2-3):275-87.

36. Umair M, Altaf M, Abbasi AM. An ethnobotanical survey of indigenous medicinal plants in Hafizabad district, Punjab-Pakistan. PloS one. 2017;12(6):e0177912.

37. Ahmed N, Mahmood A, Tahir S, Bano A, Malik RN, Hassan S, Ashraf A. Ethnomedicinal knowledge and relative importance of indigenous medicinal plants of Cholistan desert, Punjab Province, Pakistan. J Ethnopharmacol. 2014;155(2):1263-75.

38. Kayani S, Ahmad M, Sultana S, Shinwari ZK, Zafar M, Yaseen G, Hussain M, Bibi T. Ethnobotany of medicinal plants among the communities of alpine and sub-alpine regions of Pakistan. J Ethnopharmacol. 2015;164:186-202.

39. Ahmad M, Sultana S, Fazl-i-Hadi S, Ben Hadda T, Rashid S, Zafar M, Khan MA, Khan MPZ, Yaseen G. An ethnobotanical study of medicinal plants in high mountainous region of Chail valley (district swat-Pakistan). J Ethnobiol Ethnomed. 2014;10(1):36

40. Torres-Avilez W, Medeiros PMd, Albuquerque UP: Effect of gender on the knowledge of medicinal plants: systematic review and meta-analysis evidence-based complementary and alternative medicine 2016, 2016.

41. de Albuquerque UP, Soldati GT, Sieber SS, Ramos MA, de Sá JC, de Souza LC. The use of plants in the medical system of the Fulni-ô people (NE Brazil): a perspective on age and gender. J Ethnopharmacol. 2011;133(2):866-73.

42. Khan M, Khan R, Ahmed M, Muhammad N, Khan M, Khan H, Atlas N, Khan F. Biological screening of methanolic crude extracts of Caralluma tuberculata. Int J Indigenous Med Plants. 2013;46:2051-4263.

43. Amsalu N, Bezie Y, Fentahun M, Alemayehu A, Amsalu G. Use and conservation of medicinal plants by indigenous people of Gozamin Wereda, east Gojjam zone of Amhara region, Ethiopia: an ethnobotanical approach. Evid Based Complement Altern Med. 2018;2018.

44. Muhammad A, Ihsan U, Akash T, Waheed M, Azizullah A, Khan A, Nawab A. Ethnomedicine use in the war affected region of northwest Pakistan. J Ethnobiol Ethnomed. 2014:10(16).

45. Gulzar H, Hazrat A, Gulzar K, Ali F, Khan N, Nisar M, Khan I, Abid Ullah A. Medicinal plants and their traditional uses in Thana Village, District Malakand, Khyber Pakhtunkhwa, Pakistan. Int J Endorsing Health Sci Res. 2019;7(Issue 1).

46. Karunamoorthi K, Jegajeevanram K, Vijayalakshmi J, Mengistie E. Traditional medicinal plants: a source of phytotherapeutic modality in resource-constrained health care settings. J Evid Based Complement Altern Med. 2013;18(1):67-74.

47. Mafuva C, Marima-Matarira HT. Towards professionalization of traditional medicine in Zimbabwe: a comparative analysis to the South African policy on traditional medicine and the Indian Ayurvedic system. Int J Herbal Med. 2014;2(2 Part C):154-61.

48. Dweba T, Mearns M. Conserving indigenous knowledge as the key to the current and future use of traditional vegetables. Int I Inf Manag. 2011;31(6):564-71.

49. Abbasi AM, Khan MA, Shah MH, Shah MM, Pervez A, Ahmad M. Ethnobotanical appraisal and cultural values of medicinally important wild edible vegetables of lesser Himalayas-Pakistan. J Ethnobiol Ethnomed. 2013;9(1):66.

50. Aziz MA, Adnan M, Khan AH, Rehman AU, Jan R, Khan J. Ethno-medicinal survey of important plants practiced by indigenous community at Ladha subdivision, South Waziristan agency, Pakistan. J Ethnobiol Ethnomed. 2016;12(1):53.

51. Kayani S, Ahmad M, Zafar M, Sultana S, Khan MPZ, Ashraf MA, Hussain J, Yaseen $\mathrm{G}$. Ethnobotanical uses of medicinal plants for respiratory disorders among the inhabitants of Gallies-Abbottabad, northern Pakistan. J Ethnopharmacol. 2014;156:47-60.

52. Giday M, Asfaw Z, Woldu Z. Medicinal plants of the Meinit ethnic group of Ethiopia: an ethnobotanical study. J Ethnopharmacol. 2009;124(3):513-21.

53. Tugume P, Kakudidi EK, Buyinza M, Namaalwa J, Kamatenesi M, Mucunguz $P$, Kalema J. Ethnobotanical survey of medicinal plant species used by communities around Mabira central Forest reserve, Uganda. J Ethnobiol Ethnomed. 2016;12(1):5.
54. Aletor G: Domiciliary midwifery care, including traditional birth attendants. In: Maternal and child health around the world. Springer; 1981: 89-98.

55. Campero L, Garcia C, Diaz C, Ortiz O, Reynoso SA, Langer A. "alone, I wouldn't have known what to do": a qualitative study on social supportduring labor and delivery in Mexico. Soc Sci Med. 1998;47(3):395-403.

56. Napagoda MT, Sundarapperuma T, Fonseka D, Amarasiri S, Gunaratna P. An ethnobotanical study of the medicinal plants used as anti-inflammatory remedies in Gampaha District, Western Province, Sri Lanka. Scientifica. 2018;2018.

57. Abdool Karim S, Ziqubu-Page T, Arendse R. Bridging the gap: project report for the South African Medical Research Council. S Afr Med J. 1994;84:1-14.

58. Saynes-Vásquez A, Caballero J, Meave JA, Chiang F. Cultural change and loss of ethnoecological knowledge among the isthmus Zapotecs of Mexico. J Ethnobiol Ethnomed. 2013;9(1):40.

59. Amjad MS, Qaseem MF, Ahmad I, Khan SU, Chaudhari SK, Malik NZ, Shaheen H, Khan AM. Correction: descriptive study of plant resources in the context of the ethnomedicinal relevance of indigenous flora: a case study from Toli peer National Park, Azad Jammu and Kashmir, Pakistan. PloS one. 2017;12(7):e0180917.

60. Hussain A, Abbasi M, Hussain N, Majid S. A survey of important indigenous medicinal plants of district Bhimber Azad Jammu \& Kashmir, Pakistan. Int J Adv Res. 2013;1:635-44.

61. Shaheen H, Qaseem MF, Amjad MS, Bruschi P. Exploration of ethnomedicinal knowledge among rural communities of Pearl Valley; Rawalakot, district Poonch Azad Jammu and Kashmir. PLoS One. 2017;12(9):e0183956.

62. Ajaib M, Anjum M, Malik NZ, Sidiqui MF. Ethnobotanical study of some plants of Darguti, tehsil Khuiratta, Azad Jammu and Kashmir. Int J Biol Res. 2015;3(2):101-7.

63. Safeer S, Sarwar R, Ubaid-ul-Hassan KS, Anwar SMF. Exploration of ethnomedicinal flora used against various human ailments in moist temperate Himalayas of district Bagh, Azad Jammu and Kashmir. Asian J Med Pharm Res. 2017;7(1):09-15.

64. Shabir A, Naveed IR, Uneeza J, Noor UAIZ, Hina J, Farhat Y. Ethno botanical Wisdom of Inhabitant of Devi Galli Azad Kashmir. Biomed J Sci Tech Res. 2017:1(6):1618-27.

65. Ahmad KS, Habib S. Indigenous knowledge of some medicinal plants of Himalaya region, Dawarian village, Neelum valley, Azad Jammu and Kashmir Pakistan. Univ J Plant Sci. 2014;2(2):40-7.

66. Qaseem M, Qureshi R, Amjad MS, Ahmed W, Masood A, Shaheen H. Ethnobotanical evaluation of indigenous flora from the communities of rajh mehal and goi union councils of district Kotli, Azad Jammu Kashmir Pakistan. Appl Ecol Environ Res. 2019;17(2):2799-829.

67. Khan MT, Ahmad L, Rashid W. Ethnobotanical documentation of traditional knowledge about medicinal plants used by indigenous people in Talash valley of Dir lower. Northern Pakistan J Intercult Ethnopharmacol. 2018;7(1):8-24.

68. Wali R, Rahman K, Raja NI, Qureshi R. A quantitative medico-botanical expedition of fairy meadows National Park, Diamir, Gilgit Baltistan, Pakistan. BioRxiv. 2019:507848.

69. Ijaz F, Iqbal Z, Rahman IU, Alam J, Khan SM, Shah GM, Khan K, Afzal A Investigation of traditional medicinal floral knowledge of Sarban Hills, Abbottabad, KP, Pakistan. J Ethnopharmacol. 2016;179:208-33.

70. Hussain W, Badshah L, Ullah M, Ali M, Ali A, Hussain F. Quantitative study of medicinal plants used by the communities residing in Koh-e-Safaid range, northern Pakistani-afghan borders. J Ethnobiol Ethnomed. 2018;14(1):30.

71. Aziz MA, Khan AH, Adnan M, Izatullah I. Traditional uses of medicinal plants reported by the indigenous communities and local herbal practitioners of Bajaur agency, federally administrated tribal areas Pakistan. J Ethnopharmacol. 2017;198:268-81.

72. Zahoor M, Yousaf Z, Aqsa T, Haroon M, Saleh N, Aftab A, Javed S, Qadeer M, Ramazan H. An ethnopharmacological evaluation of Navapind and Shahpur Virkanin district Sheikupura, Pakistan for their herbal medicines. J Ethnobiol Ethnomed. 2017;13:27

73. Umair M, Altaf M, Bussmann RW, Abbasi AM. Ethnomedicinal uses of the local flora in Chenab riverine area, Punjab province Pakistan. J Ethnobiol Ethnomed. 2019;15(1):7

74. Fatima A, Ahmad M, Zafar M, Yaseen G, Khan MPZ, Butt MA, Sultana S. Ethnopharmacological relevance of medicinal plants used for the treatment of oral diseases in Central Punjab-Pakistan. J Herbal Med. 2018;12:88-110.

75. Mahmood A, Mahmood A, Malik RN, Shinwari ZK. Indigenous knowledge of medicinal plants from Gujranwala district, Pakistan. J Ethnopharmacol. 2013; 148(2):714-23.

76. Yabesh JM, Prabhu S, Vijayakumar S. An ethnobotanical study of medicinal plants used by traditional healers in silent valley of Kerala, India. J Ethnopharmacol. 2014;154(3):774-89. 
77. Mouterde P. New Flora of Lebanon and Syria Beirut. Catholic Printing. 1983.

78. Zia-Ul-Haq M, Ćavar S, Qayum M, Imran I, Feo VD. Compositional studies: antioxidant and antidiabetic activities of Capparis decidua (Forsk.) Edgew. Int J Mol Sci. 2011:12(12):8846-61.

79. Lulekal E, Kelbessa E, Bekele T, Yineger H. An ethnobotanical study of medicinal plants in Mana Angetu District, southeastern Ethiopia. J Ethnobiol Ethnomed. 2008;4(1):10.

80. Faruque MO, Uddin SB, Barlow JW, Hu S, Dong S, Cai Q, Li X, Hu X. Quantitative ethnobotany of medicinal plants used by indigenous communities in the Bandarban District of Bangladesh. Front Pharmacol. 2018:9:40.

81. Bibi T, Ahmad M, Tareen RB, Tareen NM, Jabeen R, Rehman SU, Sultana S, Zafar M. GY: ethnobotany of medicinal plants in district Mastung of Balochistan province-Pakistan. J Ethnopharmacol. 2014;147:79-80.

82. Agarwal K. RV: some ethnomedicinal plants of Bhopal district used for treating stone diseases. Int J Pharm Life Sci. 2012:3(13):56-62.

83. Yemele M, Telefo P, Lienou L, Tagne S, Fodouop C, Goka C, Lemfack M, Moundipa F. Ethnobotanical survey of medicinal plants used for pregnant women's health conditions in Menoua division-West Cameroon. J Ethnopharmacol. 2015;160:14-31.

84. Bano A, Ahmad M, Hadda TB, Saboor A, Sultana S, Zafar M, Khan MPZ, Arshad M, Ashraf MA. Quantitative ethnomedicinal study of plants used in the skardu valley at high altitude of Karakoram-Himalayan range, Pakistan. J Ethnobiol Ethnomed. 2014;10(1):43.

85. Basualdo I, Zardini EM, Ortiz M. Medicinal plants of Paraguay: underground organs, II. Econ Botany. 1995;49(4):387-94.

86. Noctor $\mathrm{G}$, Foyer $\mathrm{CH}$. Ascorbate and glutathione: keeping active oxygen under control. Annu Rev Plant Biol. 1998;49(1):249-79.

87. Bekele G, Reddy PR. Ethnobotanical study of medicinal plants used to treat human ailments by Guji Oromo tribes in Abaya District, Borana, Oromia, Ethiopia. Univ J Plant Sci. 2015;3(1):1-8.

88. Pascaline J, Charles M, George O, Lukhoba C. An inventory of medicinal plants that the people of Nandi use to treat malaria. J Anim Plant Sci. 2011;9:1192-200.

89. Nondo RS, Zofou D, Moshi MJ, Erasto P, Wanji S, Ngemenya MN, Titanji VP, Kidukuli AW, Masimba PJ. Ethnobotanical survey and in vitro antiplasmodial activity of medicinal plants used to treat malaria in Kagera and Lindi regions, Tanzania. J Med Plants Res. 2015;9(6):179-92.

90. Gürdal B, Kültür \$̧. An ethnobotanical study of medicinal plants in Marmaris (Muğla, Turkey). J Ethnopharmacol. 2013;146(1):113-26.

91. El Amri J, El Badaoui K, Zair T, Bouharb H, Chakir S, Alaoui T. Ethnobotanical study of medicinal plants in the region El Hajeb (Central Morocco). J Res Biol. 2015;4(8):1568-80.

92. Li W-F, Jiang J-G, Chen J. Chinese medicine and its modernization demands. Arch Med Res. 2008;39(2):246-51.

93. Boudjelal A, Henchiri C, Sari M, Sarri D, Hendel N, Benkhaled A, Ruberto G. Herbalists and wild medicinal plants in M'Sila (North Algeria): an ethnopharmacology survey. J Ethnopharmacol. 2013;148(2):395-402.

94. Luitel DR, Rokaya MB, Timsina B, Münzbergová Z. Medicinal plants used by the Tamang community in the Makawanpur district of Central Nepal. J Ethnobiol Ethnomed. 2014;10(1):5

95. Kadir MF, Sayeed MSB, Mia M. Ethnopharmacological survey of medicinal plants used by indigenous and tribal people in Rangamati, Bangladesh. J Ethnopharmacol. 2012;144(3):627-37.

96. Shaheen S, Abbas S, Hussain J, Mabood F, Umair M, Ali M, Ahmad M, Zafar M, Faroog U, Khan A. Knowledge of medicinal plants for children diseases in the environs of district Bannu, Khyber Pakhtoonkhwa (KPK). Front Pharmacol. 2017:8:430.

97. Polat R, Cakilcioglu U, Kaltalioğlu K, Ulusan MD, Türkmen Z. An ethnobotanical study on medicinal plants in Espiye and its surrounding (Giresun-Turkey). J Ethnopharmacol. 2015;163:1-11.

98. Uzun M, Kaya A. Ethnobotanical research of medicinal plants in Mihalgazi (Eskişehir, Turkey). Pharm Biol. 2016;54(12):2922-32.

99. WHO: International Classification ofPrimary Care, Second edition (ICPC-2).). 〈http://www.whoint/classifications/icd/adaptations/icpc2/en/〉 1987. (Accessed 22 Jan 2017).

100. Adzu B, Gamaniel K. Sedative effects of Cassia singueana root bark. J Nat Remedies. 2003;3(2):134-7.

101. Schlage C, Mabula C, Mahunnah R. Heinrich: medicinal plants of the Washambaa (Tanzania): documentation and ethnopharmacological evaluation. Plant Biol. 2000;2(1):83-92.

102. Ahmad M, Khan MA, Zafar M, Arshad M, Sultana S, Abbasi BH. Use of chemotaxonomic markers for misidentified medicinal plants used in traditional medicines. J Med Plants Res. 2010;4(13):1244-52.
103. Guan Y-S, He Q. Plants consumption and liver health. Evid Based Complement Alternat Med. 2015;2015.

104. Adnan M, Ullah I, Tariq A, Murad W, Azizullah A, Khan AL, Ali N. Ethnomedicine use in the war affected region of Northwest Pakistan. J Ethnobiol Ethnomed. 2014;10(1):16.

105. Sher H, Bussmann RW, Hart R, Boer HJ. Traditional use of medicinal plants among Kalasha, Ismaeli and Sunni groups in Chitral District, Khyber Pakhtunkhwa province Pakistan. J Ethnopharmacol. 2016;188:57-69.

106. Tangjitman K, Wongsawad C, Kamwong K, Sukkho T, Trisonthi C. Ethnomedicinal plants used for digestive system disorders by the Karen of northern Thailand. J Ethnobiol Ethnomed. 2015:11:27.

107. Malla B, Gauchan DP, Chhetri RB. An ethnobotanical study of medicinal plants used by ethnic people in Parbat district of western Nepal. J Ethnopharmacol. 2015;165:103-17.

108. Murad W, Azizullah A, Adnan M, Tariq A, Khan KU, Waheed S, Ahmad A. Ethnobotanical assessment of plant resources of Banda Daud Shah, district Karak, Pakistan. J Ethnobiol Ethnomed. 2013;9(1):77.

109. Albuquerque UP, Lucena RF, Monteiro JM, Florentino AT, Almeida CFC. Evaluating two quantitative ethnobotanical techniques. Ethnobot Res Appl. 2006;4:51-60.

110. Trotter RT, Logan MH. In: Etkin NL, editor. "Informant consensus: a new approach for identifying potentially effective medicinal plants," in Plants in Indigenous Medicine and Diet: Biobehavioral Approaches. New York: Redgrave Publishing Company; 1986. p. 91-112.

111. Ladio AH, Lozada M, Weigandt M. Comparison of traditional wild plant knowledge between aboriginal communities inhabiting arid and forest environments in Patagonia, Argentina. J Arid Environ. 2007:69:695-715.

112. Moerman DE. Native american ethnobotany. Portland, Oregon: timber press. Edinb J Bot. 1998;56(2):317-8.

113. Leonti $M$, Sticher $\mathrm{O}$, Heinrich M. Antiquity of medicinal plant usage in two macro-mayan ethnic groups. J Ethnopharmacol. 2003;88:119-24.

114. Leonti $\mathrm{M}$. The future is written: impact of scripts on the cognition, selection, knowledge and transmission of medicinal plant use and its implications for ethnobotany and ethnopharmacology. J Ethnopharmacol. 2011;134(3):542-55.

\section{Publisher's Note}

Springer Nature remains neutral with regard to jurisdictional claims in published maps and institutional affiliations.

\section{Ready to submit your research? Choose BMC and benefit from:}

- fast, convenient online submission

- thorough peer review by experienced researchers in your field

- rapid publication on acceptance

- support for research data, including large and complex data types

- gold Open Access which fosters wider collaboration and increased citations

- maximum visibility for your research: over $100 \mathrm{M}$ website views per year

At $\mathrm{BMC}$, research is always in progress.

Learn more biomedcentral.com/submissions 